\title{
Combined Structural ANd Manufacturing OPtimization OF STIFFENEd COMPOSITE PANELS
}

\author{
by

\section{Joseph Lynn Henderson}

Thesis submitted to the Faculty of the

Virginia Polytechnic Institute and State University

in partial fulfillment of the requirements for the degree of

\section{MASTER Of SCIENCE}

IN

AEROSPACE ENGINEERING

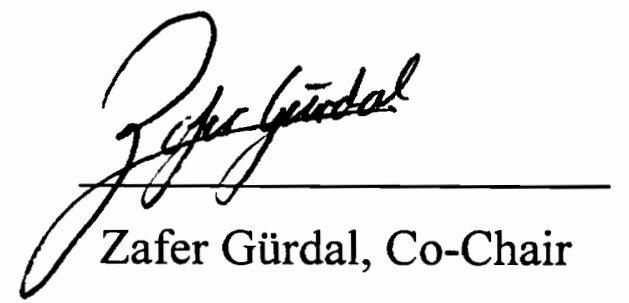

Approved:

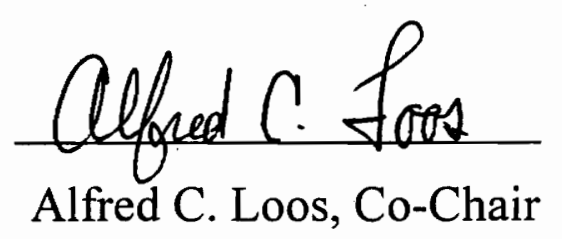

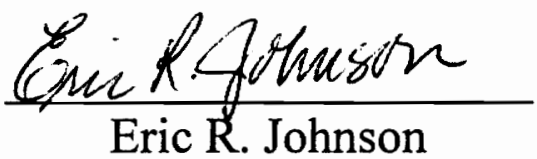

July 1996

Blacksburg, Virginia

Keywords: Composites, Structures, Manufacturing, Design Optimization, Stiffened Panels, Genetic Algorithms 
C. 2

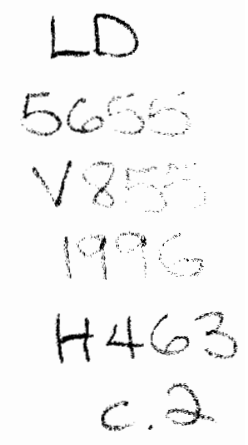




\title{
COMBINED STRUCTURAL AND MANUFACTURING OPTIMIZATION OF STIFFENED COMPOSITE PANELS
}

\author{
by
}

\author{
Joseph Lynn Henderson \\ Department of Aerospace and Ocean Engineering \\ Zafer Gürdal and Alfred C. Loos, Co-Chairmen \\ Department of Engineering Science and Mechanics
}

(ABSTRACT)

Manufacturing considerations have been incorporated into the design optimization of a blade-stiffened composite panel. For the manufacturing analysis, a one-dimensional resin film infusion model is developed to compute the infiltration time of the resin into a fabric preform of the panel. Results are presented showing the effects of structurally important design variables, such as cross-sectional geometry and material properties, on the manufacturing performance of the panel. In addition, the effects of manufacturing process variables, such as pressure and temperature, on the structural performance are studied. The structural problem is formulated to minimize the panel mass subject to buckling constraints. A simplified buckling analysis model for the panel is used to compute the critical buckling loads. The objective of the manufacturing problem is to minimize the resin infiltration time. Optimum panel designs for the manufacturing and structures problems alone, as well as for the combined problem, are generated using a genetic algorithm. These results indicate a strong connection between the structures and manufacturing design variables and trade-offs between the responses, illustrating that a multidisciplinary approach to the problem is essential to incorporating manufacturing into the preliminary design stage. 


\section{ACKNOWLEDGMENTS}

This research for this thesis was supported under the Multidisciplinary Analysis and Design Center for Advanced Vehicles at Virginia Tech. The author would also like to thank Dr. Zafer Gürdal and Dr. Alfred Loos for their assistance and direction as coadvisors to this project.

A thanks beyond words to my parents for their continuous love, support, and guidance throughout my educational career. You have been and will always be the inspiration in my life, and I dedicate this thesis to you. 


\section{TABLE OF CONTENTS}

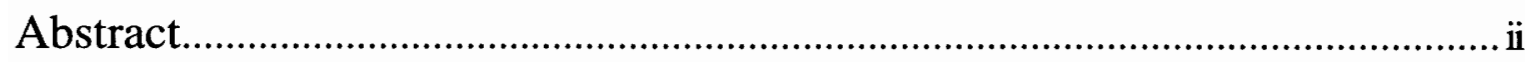

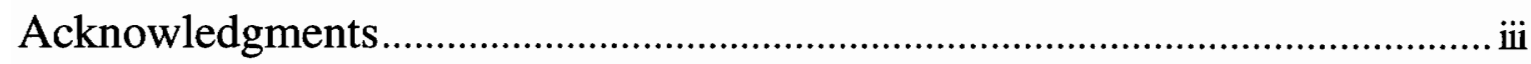

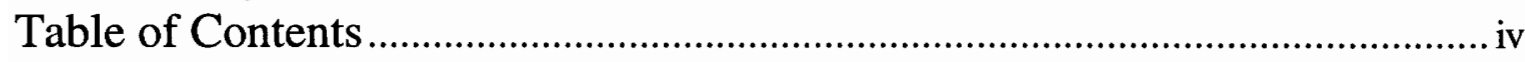

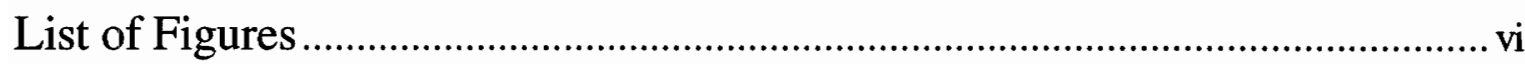

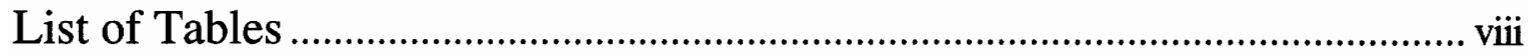

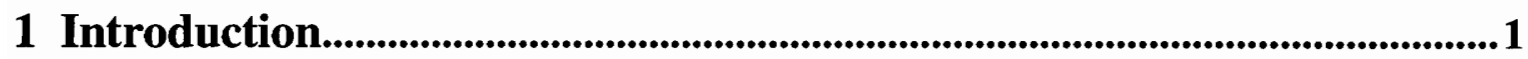

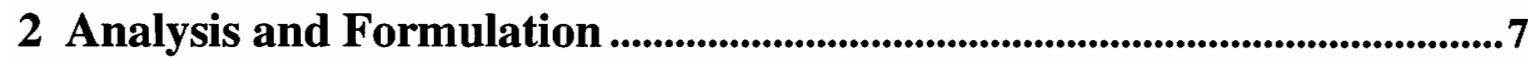

2.1 Manufacturing Analysis: Resin Film Infusion .................................................

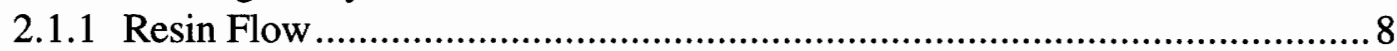

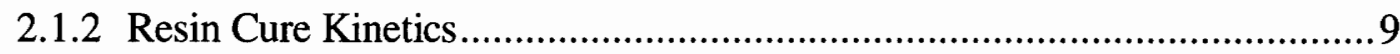

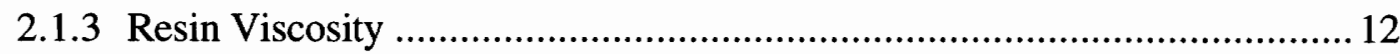

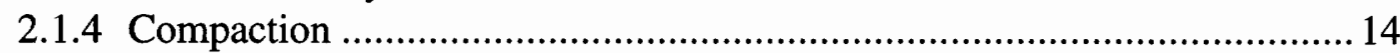

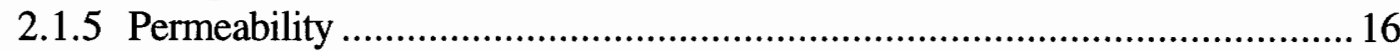

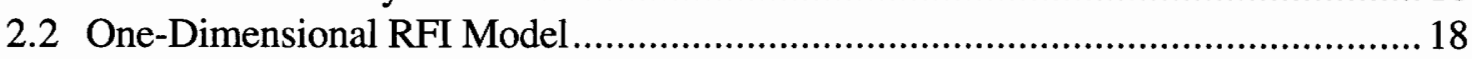

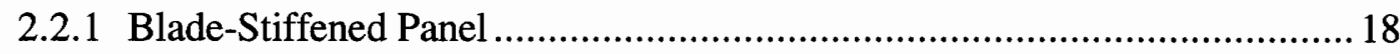

2.2.2 Panel Infiltration Time .........................................................................2 20

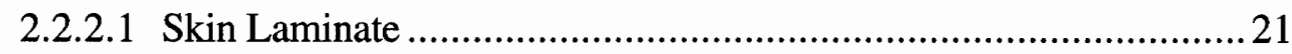

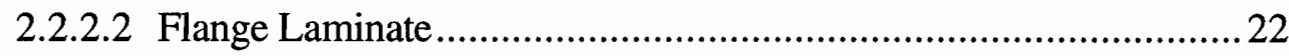

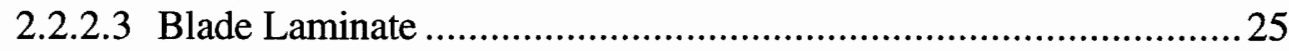

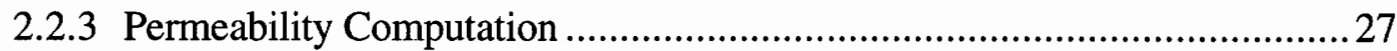

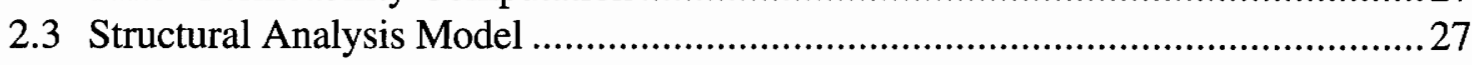

2.3.1 Micromechanics and Material Properties ..................................................30

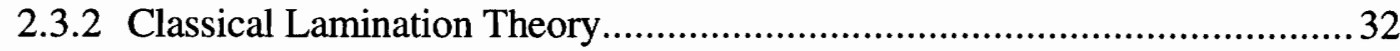

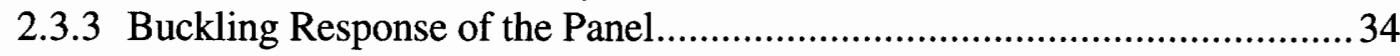

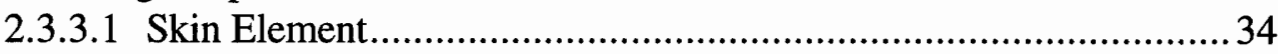

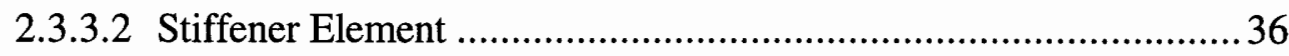

3 Optimization Problem ...........................................................................39

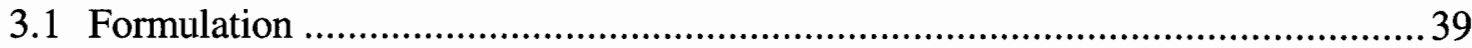

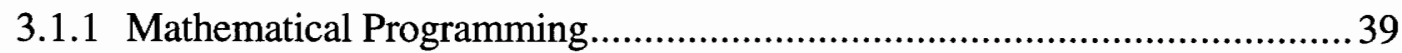

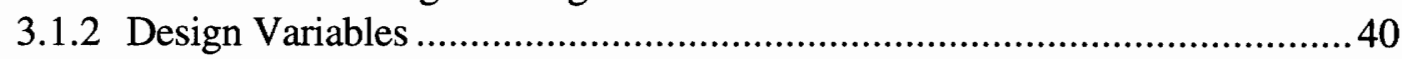

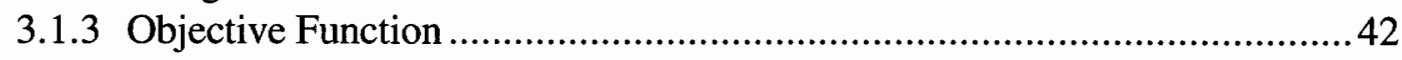

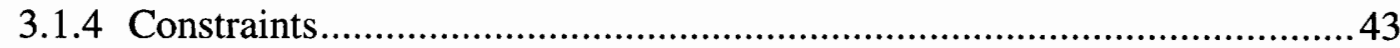

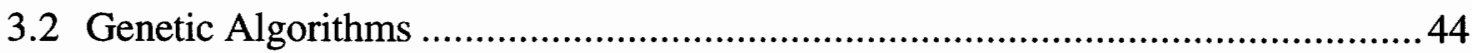

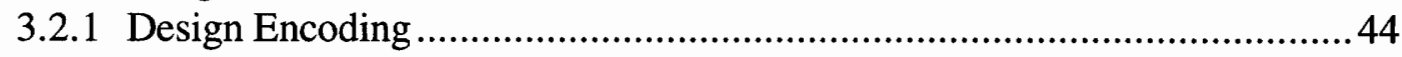

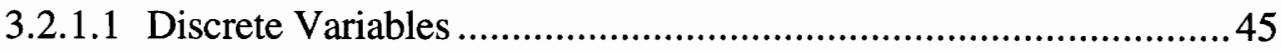

3.2.1.2 Continuous Design Variables........................................................45

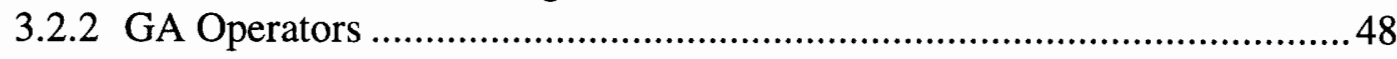




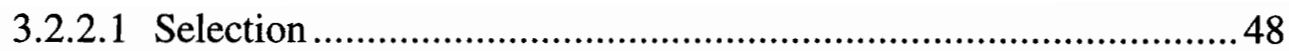

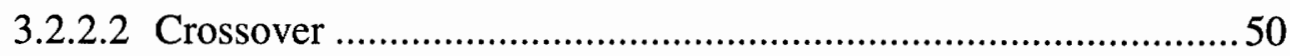

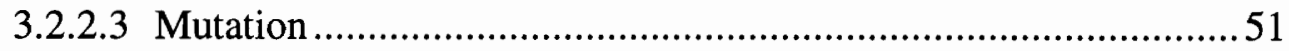

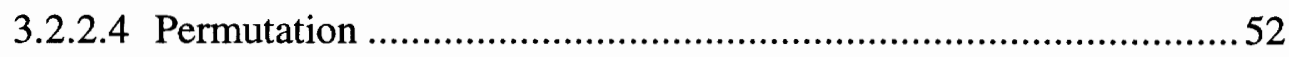

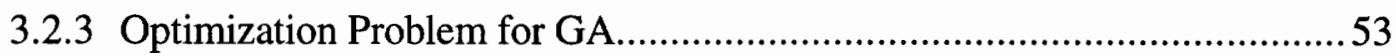

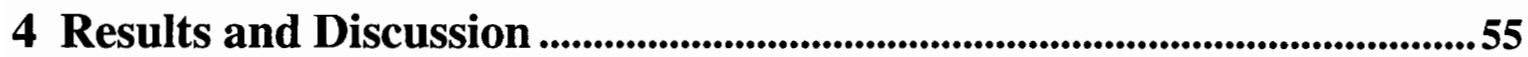

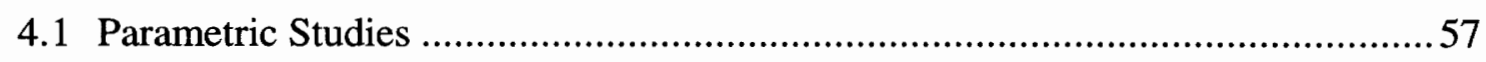

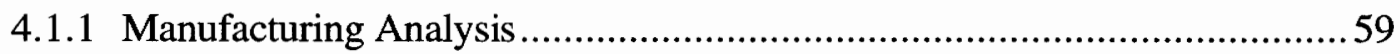

4.1.1.1 Effect of Manufacturing Variables ............................................5 59

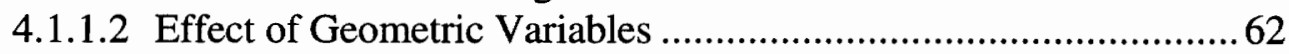

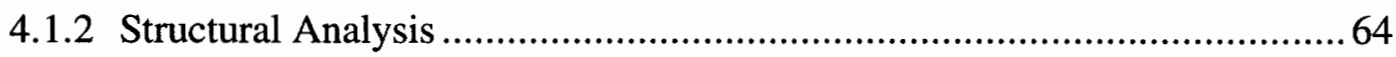

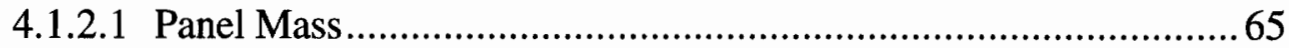

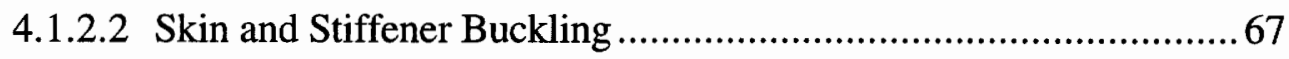

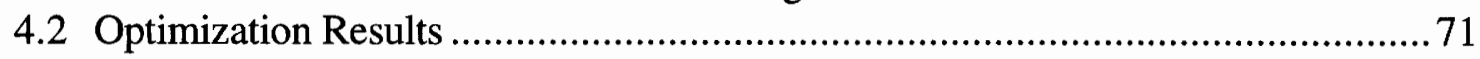

4.2.1 Role of Pressure as a Design Variable ...................................................72

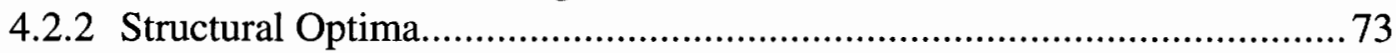

4.2.2.1 Problem Reformulation ............................................................73

4.2.2.2 Effect of Pressure Variation ...................................................... 75

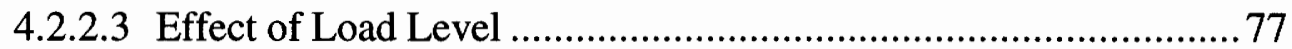

4.2.2.4 Manufacturing Analysis of Structural Optima ...............................77

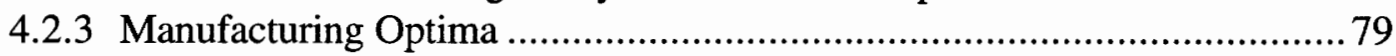

4.2.4 Combined Structural and Manufacturing Optima .......................................84

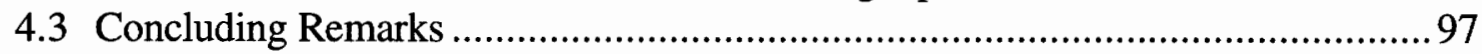

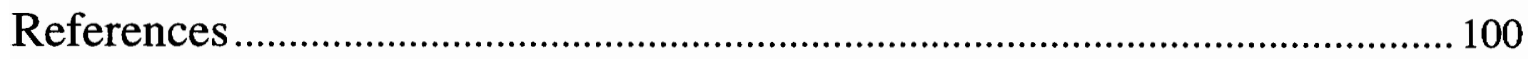

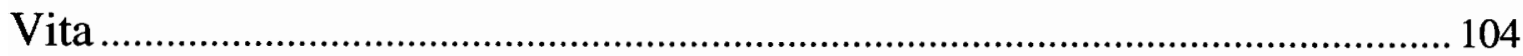




\section{LiST OF FiguRES}

Figure 2.1 Basic Setup for Resin Film Infusion.....................................................

Figure 2.2 Cure Profiles for Hercules 3501-6 Resin................................................ 11

Figure 2.3 Viscosity as a Function of Degree of Cure.................................................. 13

Figure 2.4 Compaction Pressure as a Function of AS-4 Fiber Volume Fraction ............. 15

Figure 2.5 AS-4 Laminate Ply Thickness as a Function of Fiber Volume Fraction ........ 16

Figure 2.6 Fiber Permeabilities as a Function of Compaction Pressure........................... 17

Figure 2.7 Stiffened Panel Cross-Sectional Geometry.............................................. 18

Figure 2.8 Planform of Panel with Multiple Stiffeners ................................................. 19

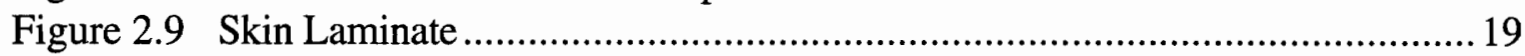

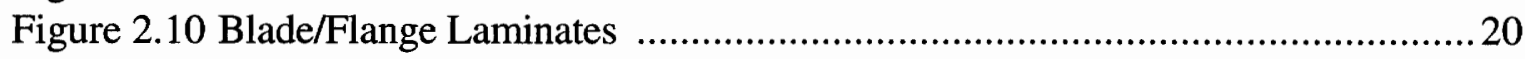

Figure 2.11 Panel Loading Condition.......................................................................2 28

Figure 2.12 Skin and Stiffener Structural Elements ....................................................29

Figure 2.13 Principal Young's Moduli as a Function of Compaction Pressure .................30

Figure 2.14 Plate Under Biaxial Compression ............................................................35

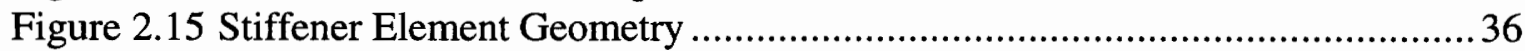

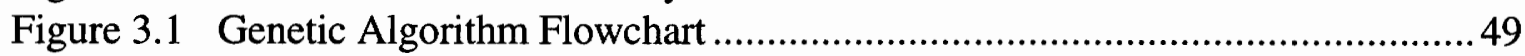

Figure 3.2 Roulette Wheel for Parent Selection ...........................................................49

Figure 3.3 Traditional Crossover Example ………....................................................50

Figure 3.4 Two-Point Crossover Example …………...............................................51

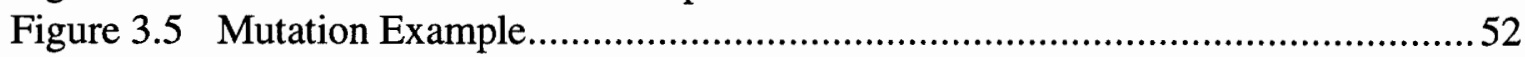

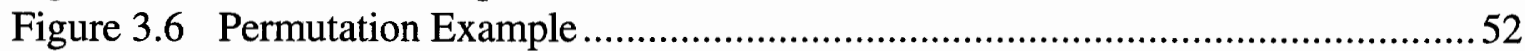

Figure 4.1 Effect of Temperature on Manufacturing ......................................................6 60

Figure 4.2 Effect of Pressure on Manufacturing ……....................................................61

Figure 4.3 Effect of Pressure on Manufacturing at Several Temperatures .....................62

Figure 4.4 Effect of Blade Height on Manufacturing ...................................................62

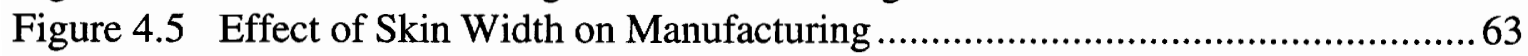

Figure 4.6 Effect of Flange-to-Skin Width Ratio on Manufacturing.................................64

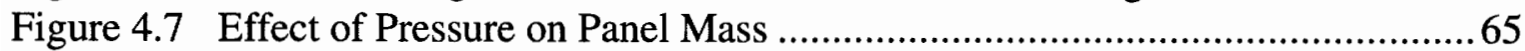

Figure 4.8 Effect of Blade Height on Panel Mass ............................................................66

Figure 4.9 Effect of Flange-to-Skin Width Ratio on Panel Mass ....................................66

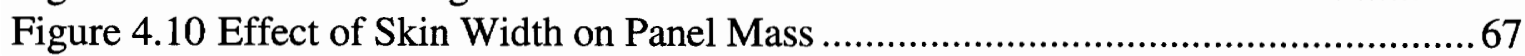

Figure 4.11 Effect of Pressure on Panel Buckling........................................................68

Figure 4.12 Effect of Blade Height on Panel Buckling..................................................69

Figure 4.13 Effect of Skin Width on Panel Buckling ................................................ 70

Figure 4.14 Effect of Flange-to-Skin Width Ratio on Panel Buckling ..............................70

Figure 4.15 Effect of Loading on Panel Buckling ....................................................... 71

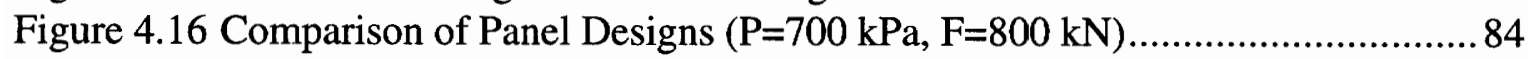

Figure 4.17 Effect of Temperature Variations in Optimization Results 


$$
(\mathrm{P}=700 \mathrm{kPa}, F=800 \mathrm{kN})
$$

Figure 4.18 Objective Trade-off for Variable Skin Width ....

Figure 4.19 Objective Trade-off for Variable Blade Height ..............................................89

Figure 4.20 Objective Trade-off for Variable Flange Width ............................................90

Figure 4.21 Objective Trade-off for Variable Pressure .................................................91

Figure 4.22 Comparison of Design Variables for Variable Pressure Combined Optima ...93

Figure 4.23 Combined Optima for Variable Compaction Pressure $(\mathrm{F}=800 \mathrm{kN}) \ldots \ldots \ldots \ldots . . . .93$

Figure 4.24 Comparison of Design Variables for Variable Loading Combined Optima ...95

Figure 4.25 Combined Optima for Variable Loading ( $\mathrm{P}=700 \mathrm{kPa})$.................................95

Figure 4.26 Structures, Manufacturing, and Combined Optima ........................................97 


\section{LIST OF TABLES}

Table 2.1 Kinetic Parameters for the Complex Cure Reaction of Hercules 3501-6....... 11

Table 4.1 Manufacturing Analyses of Minimum Weight Designs from Ref. [4]............56

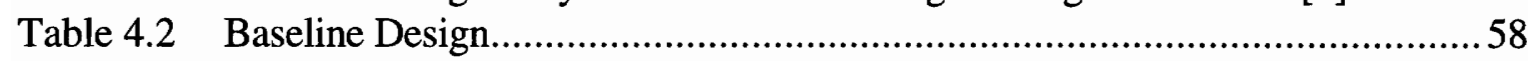

Table 4.3 Genetic Algorithm Parameters................................................................ 71

Table 4.4 Objective Function Weighting and Constraint Penalty/Bonus Parameters.....72

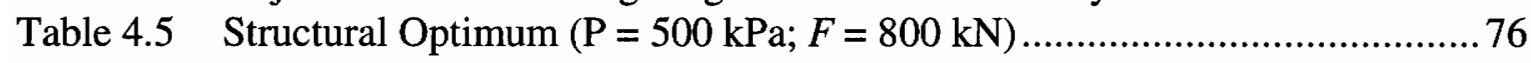

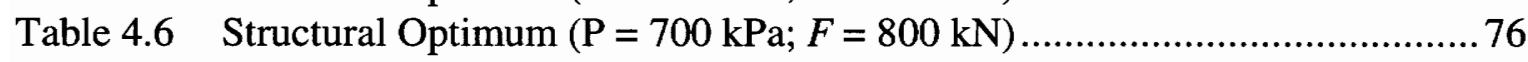

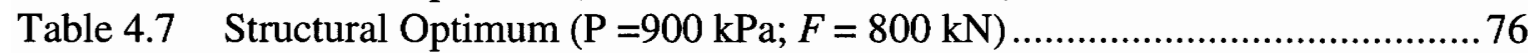

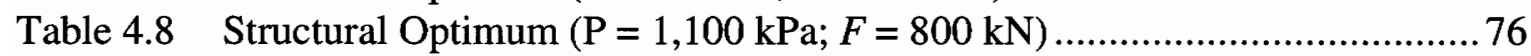

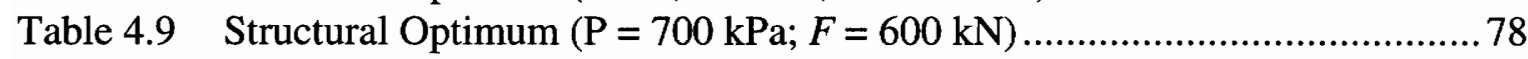

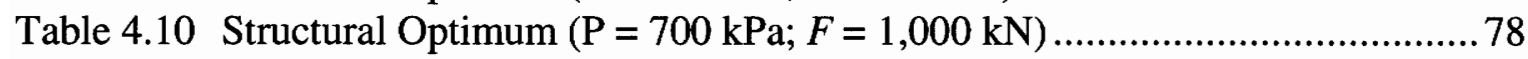

Table 4.11 Infiltration Times of Structural Optima at $T=120^{\circ} \mathrm{C}$...................................79

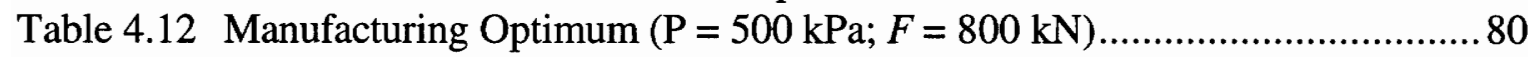

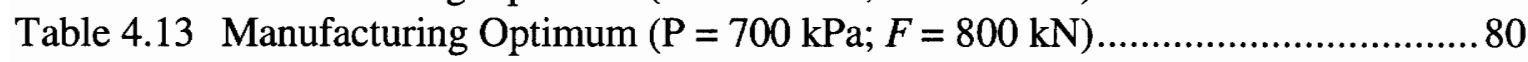

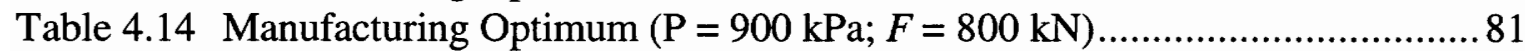

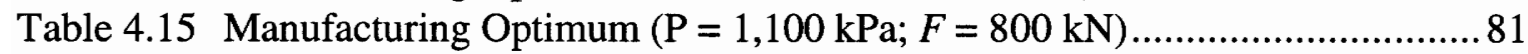

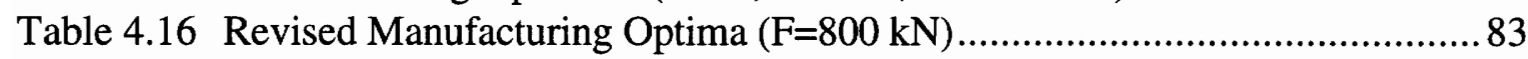

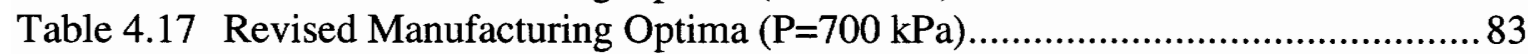

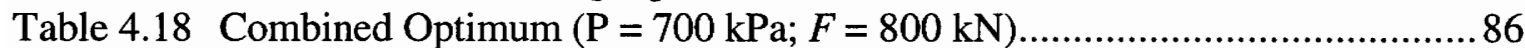

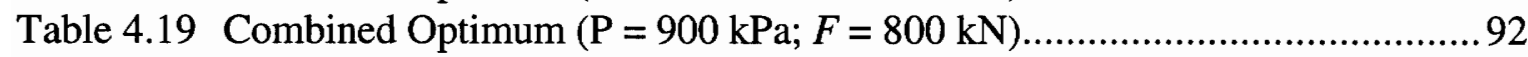

Table 4.20 Combined Optimum $(\mathrm{P}=700 \mathrm{kPa} ; F=1,000 \mathrm{kN})$ )................................... 94 


\section{INTRODUCTION}

In aircraft structural design, one of the primary goals is to minimize weight. Laminated composite materials are a natural choice as replacements for metallic structural applications, where high stiffness-to-weight ratios are required. For many aircraft applications, structural panels, such as those encountered in skins, ribs, and spars, are additionally strengthened by stiffening elements.

In recent years, the optimal design of composite stiffened panels has been widely studied [1-4]. The optimization process, however, is most often based only on the structural response of the panel. Typically, the objective is to optimize the cross-sectional geometry in order to minimize the total weight of the panel while satisfying constraints on failure loads based on strength and buckling. In addition to the geometric design variables, laminate stacking sequences in different segments of the panel are also frequently used as design variables [3-10]. Changes in these design variables influence the panel's material properties which also impact the structural performance.

One consideration, however, that has not been addressed in the research mentioned above but arises frequently, particularly for stiffened composite panels, is manufacturing. Geometries that may be optimal from a structural point of view may not lend themselves well to cost-effective manufacturing. In some cases, in fact, components of the panel geometry may be dictated solely by manufacturing considerations and existing manufacturing techniques. Moreover, materials and the manufacturing process used 
generally govern the cost of producing composite structures, which, despite their benefits and the continuous development of new, low-cost manufacturing approaches, has slowed the usage of composites in primary commercial aircraft structures [11]. This delayed acceptance of composites demonstrates the need to bring manufacturing considerations into the preliminary design phase, where the principal opportunity to achieve low-cost designs arises through the use of innovative materials, design concepts, and manufacturing technologies [12]. Boeing, for example, indicates that the majority of fabrication costs for an aircraft are fixed once preliminary designs are frozen [13].

Some attempts have been made to incorporate manufacturing into the early stages of design. Hahn [14] compares several composite manufacturing processes, including layup molding, filament winding, resin transfer molding (RTM), and pultrusion, to assess their technical and economic feasibility. Along the same lines, Falzon and Steven [15] examine the buckling responses of five stiffened panels, each manufactured by a different method. Neither of these studies, however, bring manufacturing into the design optimization process.

On the manufacturing side, the processes of RTM and resin film infusion (RFI) have recently gained considerable attention for cost-effective fabrication of stiffened composite panels [16-23]. RTM and RFI both employ a dry textile preform of the part to be manufactured. This preform is simultaneously impregnated with resin, consolidated with the addition of pressure, and cured, thereby eliminating the need for costly prepreg tape manufacture and ply lay-up. Science-based models for the simulation of such 
manufacturing processes involved in RTM and RFI have been developed for evaluating the infiltration of resin into a given structural shape such that fully consolidated and voidfree parts are obtained [20-26]. These processes include resin flow, fiber and tooling heat transfer, resin cure kinetics and viscosity, and mechanical preform compaction. However, the primary objective of these models is simply to analyze the manufacturing process for a given composite geometry and set of material properties. With the exception of some effort on the selection of a processing cycle, which involves determination of the temperature and pressure profiles as a function of the manufacturing time, no attempt is made to optimize the design itself. In fact, as will be postulated in this study, the geometry may have a strong impact on not only the manufacturing technique selected, but also on the processing variables involved, which is not addressed in the research cited above.

The tooling and curing process in the design of a composite helicopter rotor component are modeled by Lee et al. [27] in order to reduce development time and cost, a process they term "predictive manufacturing." The goal of their approach is to ascertain the effects of important process parameters such as tooling concepts, heating methods, and cure cycle definition on the manufacturing cost. Similarly, Wilson [28] discusses the selection of tooling materials based on their coefficients of thermal expansion and the resulting effects on the manufacturing and design processes. Concurrent processing of design and manufacturing information is examined by Yoshimura and Kimura [29], who discuss methodologies for construction of optimization problems where manufacturing is 
considered. Knowledge-based systems developed by Woytowitz et al. [30] and Marx et al. [31] establish a similar framework for incorporating manufacturing into the aircraft design process, but primarily from a procedural standpoint. In addition, cost models have been developed $[11,13,32-35]$ to predict the relative costs of different composite structural designs, manufacturing processes, and assembly methods in order to compare to those for equivalent metallic parts, as well as to interface with preliminary design tools. Nevertheless, as before, no effort is made in these studies to optimize designs from a manufacturing perspective.

As discussed in the literature, it is highly desirable to take a multidisciplinary approach to the design optimization problem at hand, whereby stiffened composite panels can be generated that not only achieve the desired structural response, but are also easily manufactured. It is also possible that, without much of a penalty in the structural performance, the manufacturing cost of a panel may be reduced substantially by incorporating the appropriate manufacturing analysis and constraints in the design process. Chao et al. [36] and Kristinsdottir and Zabinsky [37] take a "design-for-manufacture" approach by including manufacturing uncertainties/tolerances into optimization problem for composite panels. They account for random variations (i.e., imperfections) in panel dimensions and laminate stacking sequences that can occur in the manufacturing process. It is claimed in Refs. [36-37] that these uncertainties must be included into the design algorithm because if the imperfections in the manufacture of composite materials exceed allowable tolerances, both the material properties and the structural performance would be 
adversely affected. Nevertheless, neither of these two studies address the influence of the manufacturing process itself on the optimal panel design.

To this end, the work presented here seeks to incorporate elements of manufacturing into the design optimization of a blade-stiffened panel. It is desired to observe the effects of structurally important design variables, such as cross-sectional geometry and material properties, on the manufacturing performance of the panel design. In addition, the less obvious effects of manufacturing process variables, such as pressure and temperature, on the structural performance can be studied. For this purpose, a simplified buckling analysis model for the panel is developed to compute the structural response in terms of the panel mass and critical buckling loads. For the manufacturing response, a one-dimensional RFI model is developed to compute the infiltration time of the resin into a fabric preform of the panel. Neither of these models is computationally prohibitive to the optimization process and will allow the essential characteristics of the structural and manufacturing design problems to be studied and demonstrate the need for a multidisciplinary approach.

Chapter 2 presents an overview of the processes involved in resin film infusion, such as resin flow, resin cure kinetics, and viscosity. These processes are then integrated to develop the one-dimensional infiltration model. Chapter 2 also outlines the development of the simplified structural analysis model used. The optimization problem is defined in Chapter 3. In addition, the genetic algorithm and its application to the problem at hand are discussed. Finally, results are given in Chapter 4 illustrating the relationships 
between the structural- and manufacturing-related design variables and responses. Performance trade-offs between optimal designs are also presented in this final chapter. 


\section{ANALYSIS AND FORMULATION}

\subsection{Manufacturing Analysis: Resin Film Infusion}

The composites manufacturing process selected to be modeled is that of resin film infusion (RFI). For complex-shaped parts, such as those commonly found in aircraft structural applications, RFI is a cost-effective manufacturing technique in that its major component processes such as resin impregnation, consolidation, and cure all occur simultaneously. The basic setup of the RFI process is shown in Figure 2.1.

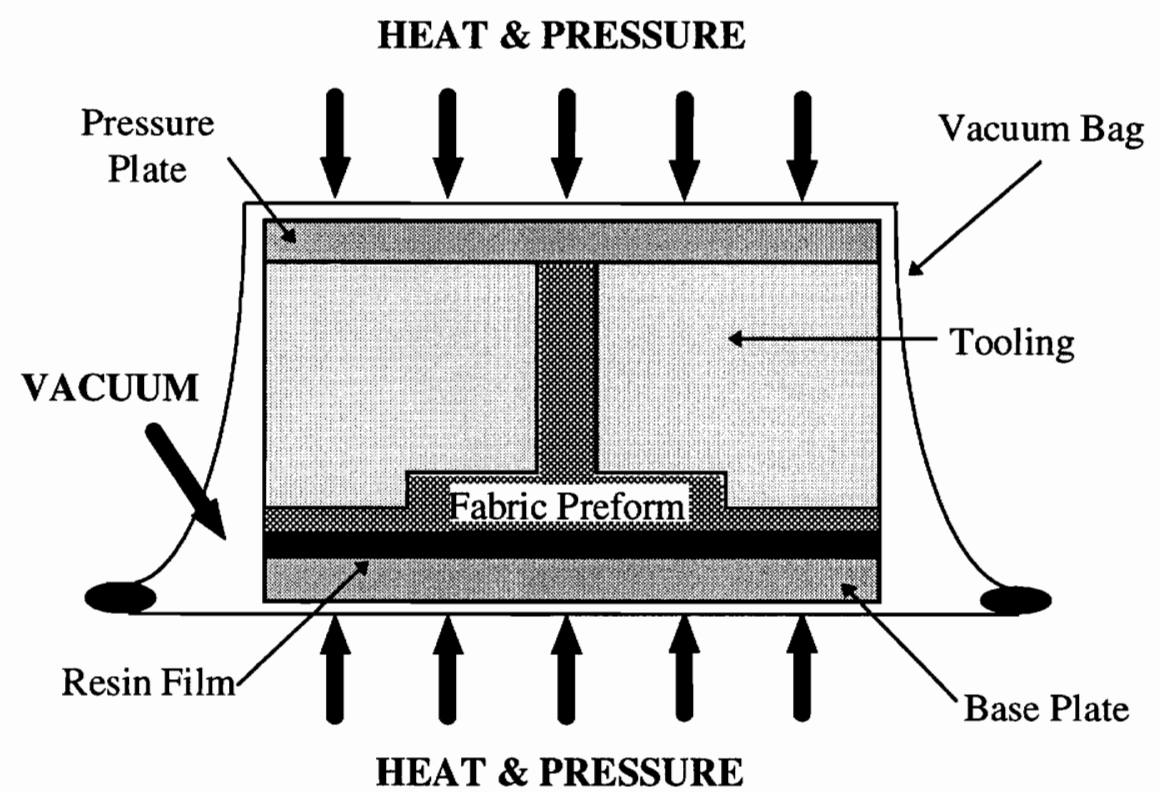

Figure 2.1 Basic Setup for Resin Film Infusion

A dry textile preform of the part to be manufactured is placed over a film of hotmelt resin. Aluminum tooling elements are used to prevent resin leakage and maintain the shape of the part. A pressure plate, typically made of graphite or aluminum, is placed on 
top of the preform and tooling. The entire assembly is covered by a vacuum bag and is then placed into an autoclave, where the resin infiltration and cure processes occur. While in the autoclave, heat and pressure are applied to the assembly and serve dual purposes. Heat not only decreases the viscosity of the resin, allowing it to flow, but also accelerates the cure of the resin-saturated part. The higher the temperature, the more quickly the resin will cure. However, if the temperature is too high, the resin may gel due to the rapid cure rate, resulting in incomplete infiltration of the preform. Pressure both drives the resin into the preform and compacts the part to its final dimensions and desired fiber volume fraction. A large pressure will force the resin into the fabric at a faster rate, but will also increase the preform's resistance to flow because of the higher degree of compaction in the fabric. Thus, temperature and pressure become the key parameters in the manufacturing analysis although, as will be demonstrated later, the geometry of the part to be filled is also important. In order to model RFI, the following processes, each of which will be discussed in further detail in the following subsections, must be considered simultaneously in the manufacturing analysis: resin flow, resin kinetics, resin viscosity, and preform compaction. Once the individual processes are introduced, they will be combined in Section 2.2 to model the RFI process for a one-dimensional representation of a stiffened panel cross-section.

\subsubsection{Resin Flow}

The flow problem is a major consideration in composite manufacturing techniques where resin is introduced into a dry textile material. For the resin film infusion model 
developed here, the flow analysis yields the governing equations for infiltration. The other analyses to be discussed in the subsequent sections will provide the necessary parameters for the solution of the flow problem.

The resin infiltration through the preform of the part can be described as flow through a homogeneous porous medium. Assuming that the flow is laminar and that the resin can be treated as an incompressible Newtonian fluid, the infiltration can be expressed by Darcy's Law [38]. The one-dimensional differential form of Darcy's Law relates $q$, the flow rate per unit area normal to the flow, to the pressure gradient as

$$
q=-\frac{S}{\eta} \frac{\partial P}{\partial z},
$$

where $P$ is the pressure, $S$ is the permeability of the preform, $\eta$ is the resin viscosity, and $z$ is the flow direction coordinate (in this case, through-the-thickness direction of the panel). The flow rate is also related to a time-dependent quantity known as the interstitial velocity $(d z / d \tau)$ by the following expression

$$
q=\phi \frac{d z}{d \tau} .
$$

Here, $\phi$ is the preform porosity and $\tau$ is the time variable. Integration of Eq. 2.2 determines the amount of time necessary for the resin to flow a certain distance.

\subsubsection{Resin Cure Kinetics}

RFI most often utilizes thermosetting resins, such as Hercules 3501-6 used in this work. The curing process involves an exothermic chemical reaction, where the amount of 
heat generated is assumed to be proportional to the rate of the cure reaction. During this reaction, a thermosetting resin will form cross-links, locking the polymer chains into a three-dimensional lattice structure that cannot be reshaped by heat. Thus, as time progresses, the resin rapidly becomes less fluid and eventually solidifies, producing the desired part. This process is quantified by the degree of cure $(\alpha)$, which is the ratio of the amount of heat evolved during the reaction up to some time $\tau$ to the total heat of reaction evolved during the entire curing process. Therefore, an uncured resin is denoted by $\alpha=0$, and $\alpha=1$ denotes a fully cured resin.

The degree of cure can be determined from a differential equation involving the rate of reaction or cure. The cure rate is a function of both degree of cure and temperature and, for thermosetting resins, can be generally expressed in the following form [22]

$$
\frac{d \alpha}{d \tau}=A \exp \left(\frac{-E}{R T}\right) f(\alpha) .
$$

Here, $A$ is a pre-exponential factor, $E$ is the Arrhenius activation energy, $R$ is the universal gas constant, $T$ is the temperature at which the resin is cured, and $f(\alpha)$ is a reaction function. The values of $A, E$, and $f(\alpha)$ are empirical and depend on the resin material.

Chiou and Letton [39] present the complex cure reaction function of Hercules $3501-6$ as the sum of three simpler ones. This kinetics model is given by the following differential equation, 


$$
\frac{d \alpha}{d \tau}=\sum_{i=1}^{3} g_{i} A_{i} \exp \left(\frac{-E_{i}}{R T}\right)(1-\alpha)^{n_{i}}
$$

where $g_{i}$ are weighting functions for each of the three reactions. Each individual reaction has its own pre-exponential factor $\left(A_{i}\right)$ and activation energy $\left(E_{i}\right)$. The reaction functions for 3501-6 are of $n_{i}^{\text {th }}$ order. All of these parameters $\left(g_{i}, n_{i}, A_{i}\right.$, and $\left.E_{i}\right)$ are resin-specific and are determined experimentally by differential scanning calorimetry. Table 2.1 [22] gives the values for Hercules 3501-6. Solving Eq. 2.4 gives $\alpha$ as a function of time, which serves as the input for the viscosity model in the next section. Plots of the cure profiles from the solution of the differential equation for several temperatures are shown in Figure 2.2. It can be seen from this figure that at low temperatures, the degree of cure increases very slowly in an almost linear fashion. At higher temperatures, however, $\alpha$ increases rapidly and then levels off, asymptotically approaching a value of one.

Table 2.1 Kinetic Parameters for the Complex Cure Reaction of Hercules 3501-6

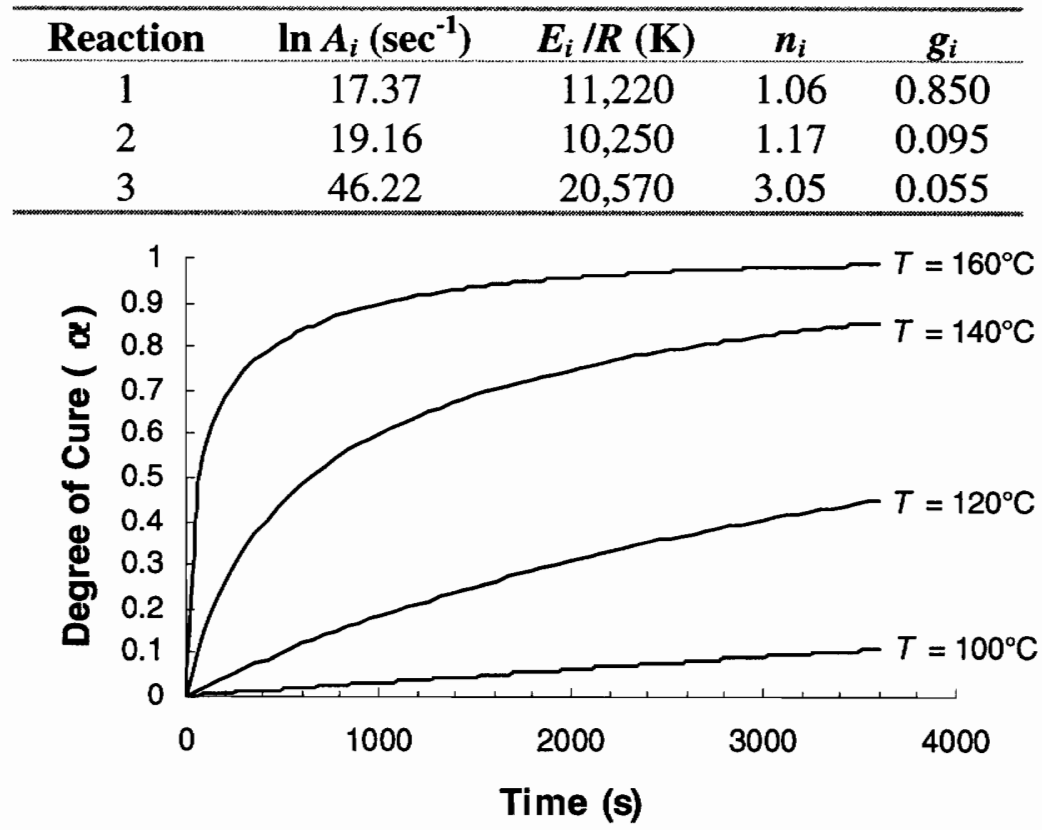

Figure 2.2 Cure Profiles for Hercules 3501-6 Resin 


\subsubsection{Resin Viscosity}

In order to compute the infiltration time of the resin into the preform, its viscosity must be known as a function of position, temperature, and time. Assuming that the resin behaves as a Newtonian fluid, an empirical relation for viscosity $(\eta)$ is governed by the Williams-Landel-Ferry (WLF) equation [40],

$$
\ln \left[\frac{\eta(T)}{\eta\left(T_{g}\right)}\right]=\frac{-C_{1}\left(T-T_{g}\right)}{C_{2}+\left(T-T_{g}\right)},
$$

where $T_{g}$ is the glass transition temperature, the temperature below which the resin is brittle and glass-like, and $C_{1}$ and $C_{2}$ are specified constants. Both the glass transition temperature model and the viscosity at $T_{g}, \eta\left(T_{g}\right)$, are resin-specific and depend on the degree of cure $(\alpha)$. Based on experimental data for Hercules 3501-6, Chiou and Letton [39] provide the following polynomial functions of $\alpha$ for the two quantities. The glass transition temperature is a fifth-order polynomial,

$$
T_{g}(\alpha)=283.42+196.4 \alpha-925.4 \alpha^{2}+3435 \alpha^{3}-4715 \alpha^{4}+2197 \alpha^{5},
$$

and the $\eta\left(T_{g}\right)$ relation is given as a third-order polynomial,

$$
\ln \left[\eta\left(T_{g}\right)\right]=20.72+8.56 \alpha-9.69 \alpha^{2}+41.17 \alpha^{3}=H(\alpha) \text {. }
$$

The values of the constants are also resin-dependent and have the values $C_{1}=29.667$ and $C_{2}=36.926$ for $3501-6[22]$.

Solving Eq. 2.5 for viscosity as a function of cure temperature and degree of cure yields the following expression, 


$$
\eta(T, \alpha)=\exp \left\{\frac{-C_{1}\left[T-T_{g}(\alpha)\right]}{C_{2}+\left[T-T_{g}(\alpha)\right]}+H(\alpha)\right\}
$$

As alluded to in Section 2.1, as the degree of cure increases, the viscosity also increases. It is assumed in the present study that when the resin viscosity reaches $1,000 \mathrm{~Pa} \cdot \mathrm{s}$, the resin is considered to have gelled, or solidified to the point that flow is no longer possible. Figure 2.3 shows the viscosity as a function of cure and temperature.

It is desired to have the preform completely infiltrated prior to the resin gelling. If not, the part will contain voids that will degrade its structural performance. It is clear from the plot that as the temperature increases, the resin will reach a higher degree of cure before the resin gels. Recall from Eq. 2.4, the degree of cure and thus the viscosity are time-dependent quantities.

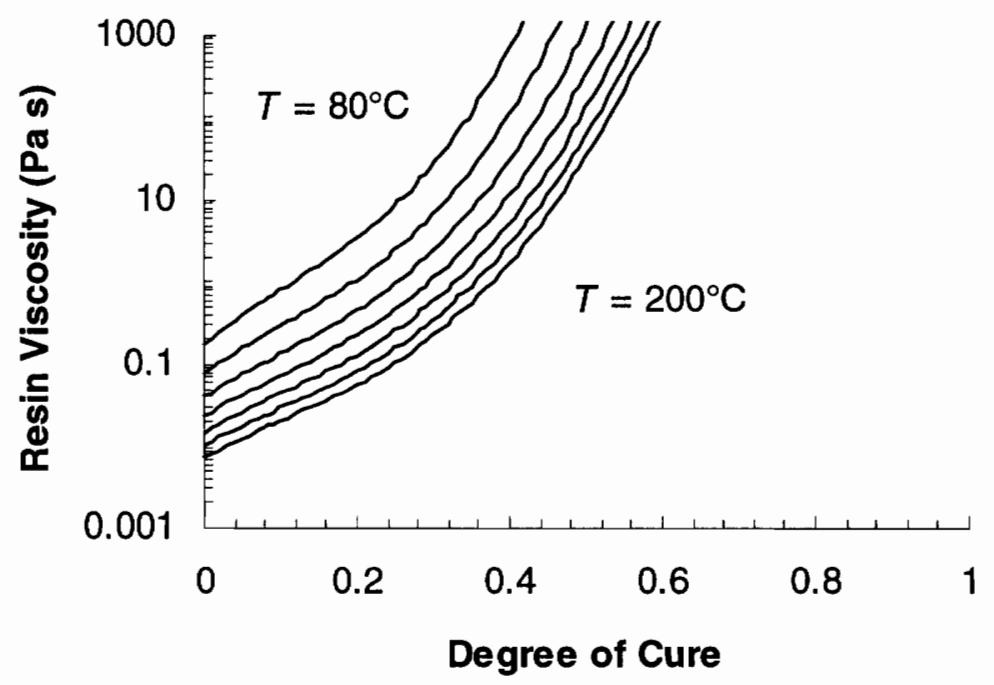

Figure 2.3 Viscosity as a Function of Degree of Cure 


\subsubsection{Compaction}

In the RFI process, pressure is necessary to drive the resin into the preform, compact the preform to its required dimensions and increase the fiber volume fraction by reducing the air pockets left in the preform. The compaction pressure is not only directly tied to the flow rate in Darcy's Law, but also indirectly by way of the porosity. The fiber volume fraction $\left(v_{f}\right)$ of the composite is related to the porosity as follows

$$
v_{f}=1-\phi .
$$

Gutowski et al. [41] developed a relationship between the fiber volume fraction and the compaction pressure. The pressure required for consolidation is written as

$$
P=K \frac{\sqrt{\frac{v_{f}}{v_{0}}}-1}{\left(\sqrt{\frac{v_{\max }}{v_{f}}}-1\right)^{4}},
$$

where $v_{0}$ is the initial fiber volume fraction, $v_{\max }$ is the maximum allowable fiber volume fraction which depends on the fiber packing arrangement, and $K$ is a constant based on the fiber material. For AS-4 carbon fibers, $K=0.159 \mathrm{kPa}, v_{0}=0.51$, and $v_{\max }=0.829$ [41]. Eq. 2.10 for AS-4 fibers is plotted in Figure 2.4. From this relation, it can be seen that when $P=0, v_{f}=v_{0}$, and as $P \rightarrow \infty, v_{f} \rightarrow v_{\max }$.

For the model to be used in this work, it is desired to compute the fiber volume fraction of the preform given the applied pressure. However, the inverse relation of Eq. 
2.10 is not easily obtained. To this end, a logarithmic regression model is computed to achieve the desired functional relation,

$$
\mathrm{v}_{f}=0.540 P^{0.0416}
$$

This expression requires $P$ in $\mathrm{kPa}$.

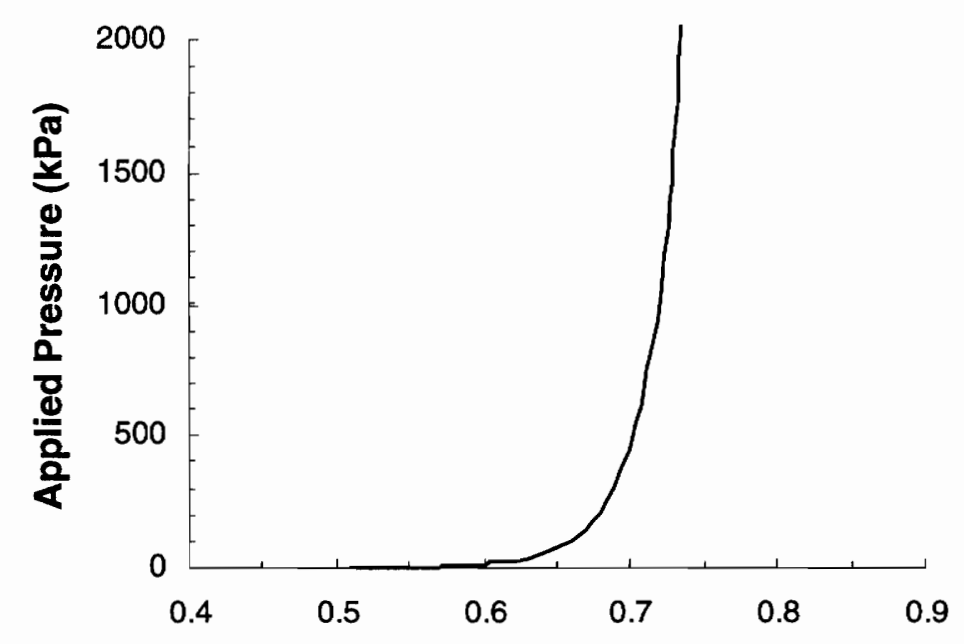

Fiber Volume Fraction $\left(v_{t}\right)$

Figure 2.4 Compaction Pressure as a Function of AS-4 Fiber Volume Fraction

Once the fiber volume fraction is known, the thickness of an individual ply can be computed as follows,

$$
t_{p l y}=\frac{\xi}{\rho_{f} v_{f}},
$$

where $\xi$ is the areal weight of the fiber and $\rho_{f}$ is the fiber density. For AS-4 fibers, these values are $\xi=0.152 \mathrm{~kg} / \mathrm{m}^{2}$, and $\rho_{f}=1.79 \times 10^{3} \mathrm{~kg} / \mathrm{m}^{3}$. Eq. 2.12 is plotted in Figure 2.5 . 


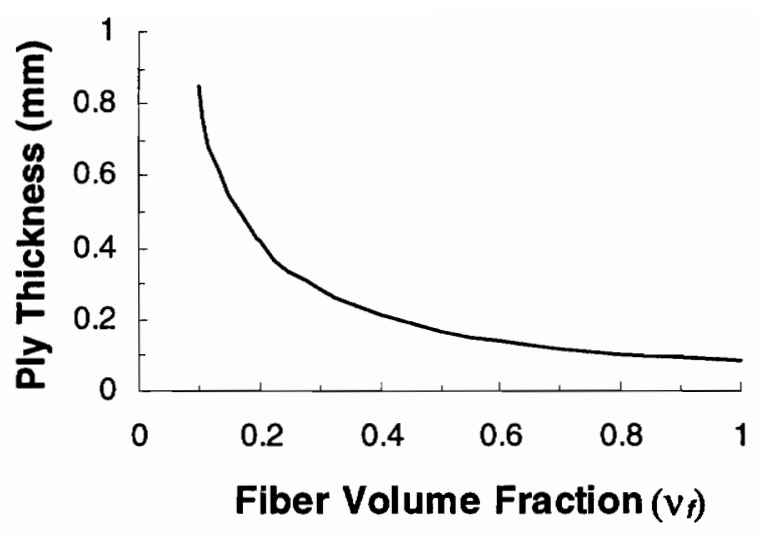

Figure 2.5 AS-4 Laminate Ply Thickness as a Function of Fiber Volume Fraction

The ply thickness is an important quantity in both the manufacturing and structural analyses, and Eq. 2.12 provides a relationship $t_{p l y}$ and the compaction pressure, by way of $v_{f}$

\subsubsection{Permeability}

The last element that factors into Darcy's Law is the permeability of the preform. Permeability is a measure of the preform resistance to resin flow and is dependent on the fiber radius, the porosity/fiber volume fraction, the packing arrangement of the fibers, and the fiber direction. The permeability along the fiber direction is given by the KozenyCarman Equation [42],

$$
S_{11}=\frac{r^{2} \phi^{3}}{4 K_{1}(1-\phi)^{2}}
$$

where $r$ is the fiber radius, taken to be $4 \mu \mathrm{m}$, and $K_{1}$ is a constant with the value of 0.7 .

The permeability perpendicular to the fibers is computed by Gebart [43] as follows, 


$$
S_{22}=K_{2} r^{2}\left(\sqrt{\frac{v_{\max }}{v_{f}}-1}\right)^{\frac{5}{2}} .
$$

$K_{2}$ is a constant with the value of 0.231 , and $v_{\max }$ is the maximum allowable fiber volume fraction. Including the relations in Eqs. 2.9 and 2.11, $S_{11}$ and $S_{22}$ can be plotted as functions of pressure as shown in Figure 2.6. For "off-axis" permeabilities, a coordinate transformation [23] is used. Establishing a coordinate system such that $0^{\circ}$ fibers are perpendicular to the flow direction and $90^{\circ}$ fibers are parallel to the flow direction, the permeability for an angle $\theta$ is given by

$$
S_{11} \sin ^{2}(\theta)+S_{22} \cos ^{2}(\theta) .
$$

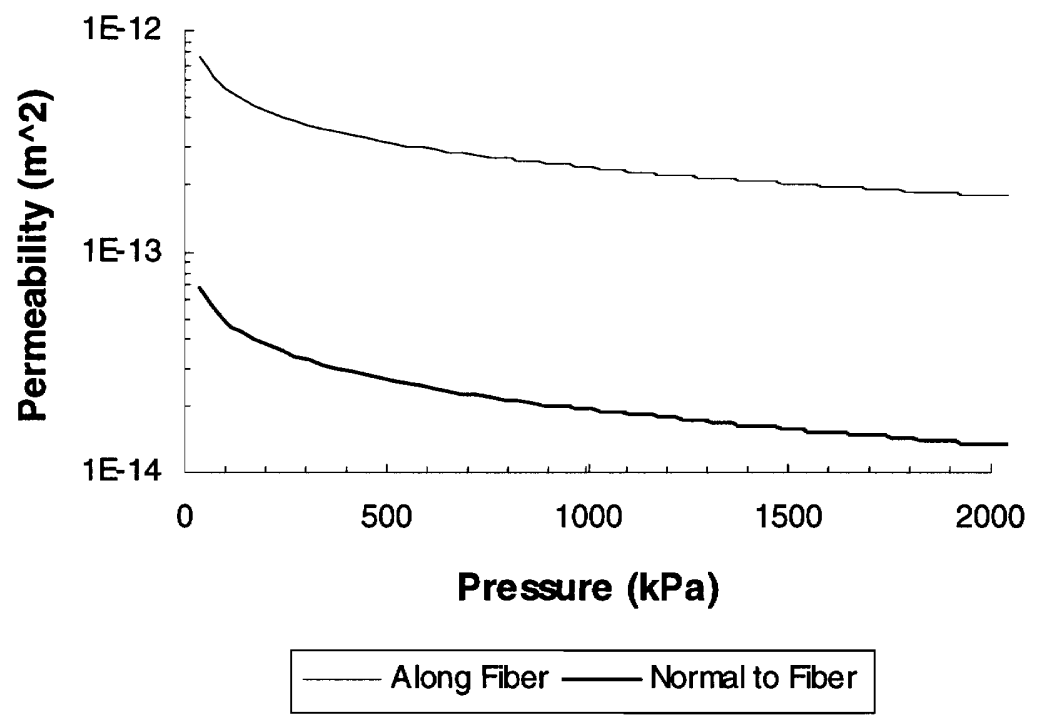

Figure 2.6 Fiber Permeabilities as a Function of Compaction Pressure 


\subsection{One-Dimensional RFI Model}

Encompassing the physical processes of RFI discussed above, a one-dimensional flow model has been developed to compute the resin infiltration time of a blade-stiffened panel.

\subsubsection{Blade-Stiffened Panel}

The panel under consideration is shown in Figure 2.7. The panel consists of a skin laminate and blade and flange laminates, which make up the stiffener.

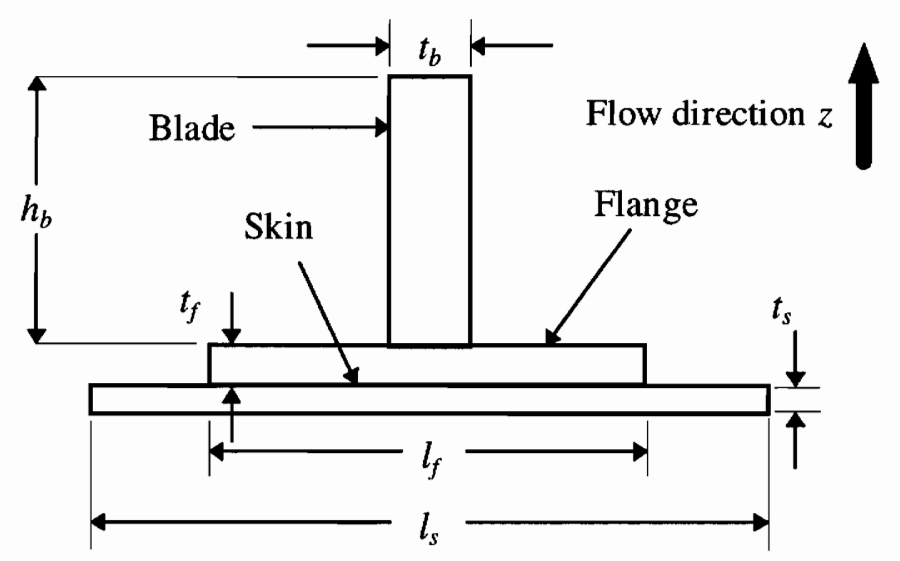

Figure 2.7 Stiffened Panel Cross-Sectional Geometry

The variables $t_{s}, t_{f}$, and $t_{b}$ represent the skin, flange, and blade thicknesses, respectively. The skin width, flange width, and blade height are denoted by $l_{s}, l_{f}$, and $h_{b}$, respectively. For structural applications, several unit cells (Figure 2.7) typically comprise the stiffened panel as shown in Figure 2.8. The overall dimensions of the panel are $W \times L$. 


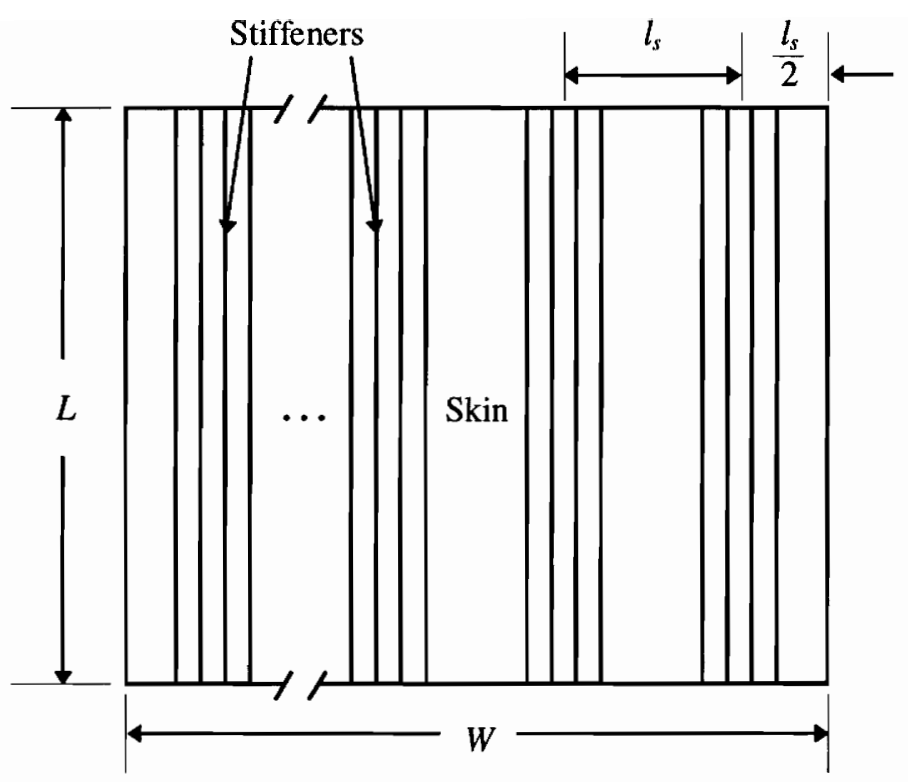

Figure 2.8 Planform of Panel with Multiple Stiffeners

The skin and blade laminates of the panel are composed of $N_{s}$ and $N_{b}$ plies, respectively, of thickness $t_{p l y}$, as determined by Eq. 2.12. The fiber orientations are assumed to be either $0^{\circ}, \pm 45^{\circ}$, or $90^{\circ}$. The skin and blade laminates are also assumed to be symmetric about the mid-plane and balanced. The balanced condition states that for every positive fiber orientation, there is a corresponding negative fiber orientation. A representation of the skin laminate alone is shown in Figure 2.9.

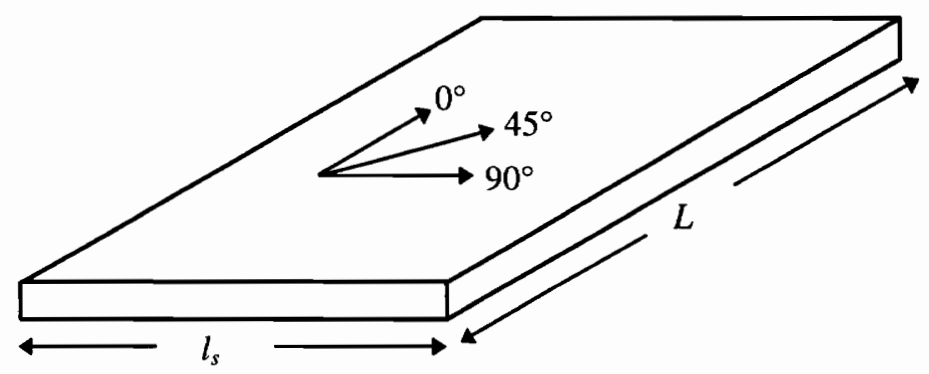

Figure 2.9 Skin Laminate 
The flange laminate is obtained by splitting the bottom portion of the blade laminate in half. Thus, the flange thickness $\left(t_{f}\right)$ is one-half of the blade thickness $\left(t_{b}\right)$. Although the flange laminate is balanced, as will be explained in Section 3.1.2, it is not symmetric like the skin and blade laminates. The stiffener element is shown in Figure 2.10. The figure also illustrates the $90^{\circ}$ fiber direction in both the blade and flange. The $0^{\circ}$ fiber direction is along the length of the stiffener.

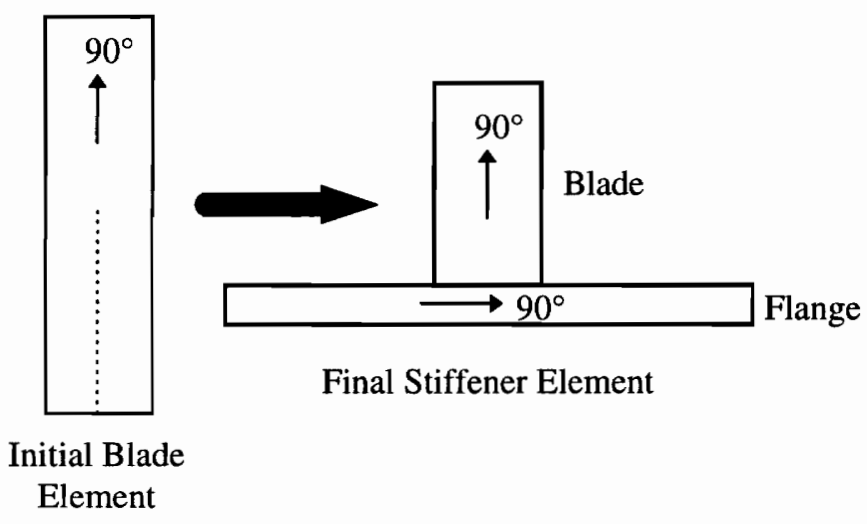

Figure 2.10 Blade/Flange Laminates

\subsubsection{Panel Infiltration Time}

The infiltration time equations for the skin, flange, and blade portions of the panel are developed following the procedure outlined by Cai and Lawrie [44]. It is assumed that the resin flows uniformly into the cross-section shown in Figure 2.7 from the base of the panel such that one-dimensional flow is always maintained along the through-the-thickness direction.. The infiltration times for each panel section are obtained by integrating Darcy's Law (Eq. 2.1) and applying the continuity equation, which states that the mass flow rate must remain constant throughout the panel. The subscripts $s, f$, and $b$ will be used to 
represent the skin, flange, and blade, respectively. Double subscripts will denote the conditions at section boundaries (e.g., $s f$ denotes the skin/flange interface). For simplicity, the following through-the-thickness locations in the panel (see Figure 2.7) are defined,

$$
\begin{aligned}
& z_{1}=t_{s} \\
& z_{2}=t_{s}+t_{f} \\
& z_{3}=t_{s}+t_{f}+h_{b}
\end{aligned}
$$

\subsubsection{Skin Laminate}

From Darcy's Law (Eq. 2.1), the flow rate is given by

$$
q_{s}=-\frac{S_{s}}{\eta} \frac{d P}{d z},
$$

where $S_{s}$ is the skin permeability to be defined in Section 2.2.3. Integrating this expression, letting the pressure at the inlet (i.e., the compaction pressure) be $P$, which is assumed to remain constant, and assuming that the pressure at the flow front $\left(P_{F F}\right)$ is zero, the relationship between flow rate and pressure becomes

$$
q_{s}(\tau)=\left(P-P_{F F}\right) \frac{S_{s}}{\int_{0}^{z} \eta(\tau) d z}=\frac{P S_{s}}{\int_{0}^{z} \eta(\tau) d z},
$$

where $z$ denotes an arbitrary flow front location in the skin. Note that the viscosity and flow rates are both functions of time $(\tau)$. The reason that the viscosity term is left within the $z$-coordinate integral will be explained at the end of this subsection. The implication of Eq. 2.18 is that, under constant pressure $P$ at the inlet, the flow rate reduces as the flow penetrates the cross-section. 
Recalling the time dependency expression from Eq. 2.2, the following differential equation is obtained from Eq. 2.18,

$$
q_{s}(\tau)=\phi \frac{d z}{d \tau}=\frac{P S_{s}}{\int_{0}^{z} \eta(\tau) d z}
$$

Solving Eq. 2.19, the time $\left(\tau_{s}\right)$ to infiltrate the skin portion of the preform becomes

$$
\tau_{s}=\frac{\phi}{P S_{s}} \int_{0}^{z_{1}}\left(\int_{0}^{z} \eta(\tau) d z\right) d z .
$$

In this equation, and also in the flange and blade equations that follow, the viscosity is left within an integral expression in $z$ because it varies with time, and subsequently location. In the computation, these integrals are divided into smaller intervals over which the viscosity is taken to be piecewise constant. The time for the resin to infiltrate that small interval is then determined. Using this time, in addition to the time that has already elapsed, the degree of cure of the resin is determined from Eq. 2.4. Knowing this value, a new viscosity can be computed from Eq. 2.8 to calculate the infiltration time for the next interval.

\subsubsection{Flange Laminate}

The resin flow rate in the flange is obtained by once again integrating Darcy's Law,

$$
q_{f}=-\frac{S_{f}}{\eta} \frac{d P}{d z} .
$$


The flange permeability $\left(S_{f}\right)$ will be defined in Section 2.2.3. The limits of integration are from the skin/flange interface at $z_{1}$, where the pressure is denoted by $P_{s f}$, to some arbitrary location $z$, where the flow front pressure $\left(P_{F F}\right)$ is again assumed to be zero. Thus, similar to Eq. 2.17 , the relationship between $q_{f}$, which again is a function of $\tau$, and $P_{s f}$ is given by

$$
q_{f}(\tau)=\left[P_{s f}(\tau)-P_{F F}\right] \frac{S_{f}}{\int_{z_{1}}^{z} \eta(\tau) d z}=\frac{P_{s f}(\tau) S_{f}}{\int_{z_{1}}^{z} \eta(\tau) d z} .
$$

$P_{s f}$ serves as the inlet pressure for the flange section and is an unknown, time-varying quantity. In order to relate $P_{s f}$ to the known compaction pressure $P$, the skin flow rate must again be considered.

When the resin moves from the skin to flange portion of the panel, there is also a change in the flow area. This change subsequently affects the resin flow rate and must be accounted for through the continuity equation,

$$
q_{s}(\tau) A_{s}=q_{f}(\tau) A_{f}=q_{b}(\tau) A_{b}=\text { constant },
$$

where $A_{s}, A_{f}, A_{b}$ are the flow areas of the skin, flange and blade, respectively, and $q_{b}$ is the resin flow rate in the blade portion of the panel. Integrating Eq. 2.17 from the inlet of the skin to the inlet of the flange, the skin flow rate can be expressed as a function of both $P$ and $P_{s f}$ as follows,

$$
q_{s}(\tau)=\left[P-P_{s f}(\tau)\right] \frac{S_{s}}{\int_{0}^{z_{1}} \eta(\tau) d z}
$$


By substituting the above relation for $q_{s}$ into the continuity equation (Eq. 2.23), an alternate form of the flange flow rate can be obtained. Since the panel length dimension is constant, flow area ratios can also be expressed in terms of the skin and flange widths and the blade thickness $\left(l_{s}, l_{f}\right.$, and $\left.t_{b}\right)$. Thus, the flange flow rate is

$$
q_{f}(\tau)=\frac{A_{s}}{A_{f}} q_{s}(\tau)=\frac{l_{s}}{l_{f}} q_{s}(\tau)=\frac{l_{s}}{l_{f}}\left[P-P_{s f}(\tau)\right] \frac{S_{s}}{\int_{0}^{z_{1}} \eta(\tau) d z}
$$

This relation serves as the boundary condition for the flange section. For convenience, the following time-varying quantity that appears in Eq. 2.24-25 is defined,

$$
C_{s f}(\tau)=\frac{1}{S_{s}} \int_{0}^{z_{1}} \eta(\tau) d z \frac{l_{f}}{l_{s}}
$$

Incorporating Eq. 2.22 along with $C_{s f}$, the unknown $P_{s f}$ can be eliminated from Eq. 2.25. This yields

$$
q_{f}(\tau)=\frac{P-P_{s f}(\tau)}{C_{s f}(\tau)}=\frac{1}{C_{s f}(\tau)}\left(P-q_{f}(\tau) \frac{1}{S_{f}} \int_{z_{1}}^{z} \eta(\tau) d z\right)
$$

Solving for $q_{f}$ and including time dependency from Eq. 2.2, the flow rate in the flange in terms of the compaction pressure alone is

$$
q_{f}(\tau)=\frac{P}{C_{s f}(\tau)+\frac{1}{S_{f}} \int_{z_{1}}^{z} \eta(\tau) d z}=\phi \frac{d z}{d \tau}
$$

Solving this differential equation, the time for the resin to infiltrate the flange is 


$$
\tau_{f}=\frac{\phi}{P} \int_{z_{1}}^{z_{2}}\left(\frac{1}{S_{s}} \int_{0}^{z_{1}} \eta(\tau) d z \frac{l_{f}}{l_{s}}+\frac{1}{S_{f}} \int_{z_{1}}^{z} \eta(\tau) d z\right) d z
$$

\subsubsection{Blade Laminate}

The derivation of the resin infiltration time in the blade follows a similar procedure to that of the flange. Darcy's Law for the blade portion of the panel can be written as

$$
q_{b}=-\frac{S_{b}}{\eta} \frac{d P}{d z}
$$

Integrating Eq. 2.30 from $z_{2}$ with unknown, time-varying pressure $P_{f b}$ to the flow front with zero pressure, the blade flow rate is

$$
q_{b}(\tau)=\left[P_{f b}(\tau)-P_{F F}\right] \frac{S_{b}}{\int_{z_{2}}^{z} \eta(\tau) d z}=\frac{P_{f b}(\tau) S_{b}}{\int_{z_{2}}^{z} \eta(\tau) d z}
$$

As with the other permeabilities, Section 2.2 .3 will define $S_{b} . \quad P_{f b}$ serves as the inlet pressure of the blade section and must be expressed in terms of known quantities.

Once again, a boundary condition is developed from the flow rate in the previous section (i.e., the flange and the skin). An alternate expression for the flange flow rate as a function of both the flange and blade inlet pressures is obtained through integration of Eq. 2.21 from $z_{1}$ to $z_{2}$,

$$
q_{f}(\tau)=\left[P_{s f}(\tau)-P_{f b}(\tau)\right] \frac{S_{f}}{\int_{z_{1}}^{z_{2}} \eta(\tau) d z}
$$

Incorporating this relation into the continuity equation (Eq. 2.23), the flow rate in the blade can also be expressed by 


$$
q_{b}(\tau)=\frac{A_{f}}{A_{b}} q_{f}(\tau)=\frac{l_{f}}{t_{b}} q_{f}(\tau)=\frac{l_{f}}{t_{b}}\left[P_{s f}(\tau)-P_{f b}(\tau)\right] \frac{S_{f}}{\int_{z_{1}}^{z_{2}} \eta(\tau) d z}
$$

Eq. 2.33 contains two unknown pressures, $P_{s f}$ and $P_{f b}$, which must be eliminated.

If Eq. 2.32 is equated to the first relation in Eq. 2.27, an expression for $P_{s f}$, independent of flow rates, can be determined,

$$
P_{s f}(\tau)=\frac{P \int_{z_{1}}^{z_{2}} \eta(\tau) d z+C_{s f}(\tau) P_{f b}(\tau) S_{f}}{\int_{z_{1}}^{z_{2}} \eta(\tau) d z+C_{s f}(\tau) S_{f}} .
$$

Substituting this relation into Eq. 2.33 and simplifying the resulting expression, the blade flow rate becomes

$$
q_{b}(\tau)=\frac{P-P_{f b}(\tau)}{C_{f b}(\tau)}
$$

where $C_{f b}(\tau)$ is defined as

$$
C_{f b}(\tau)=\left(C_{s f}(\tau)+\frac{1}{S_{f}} \int_{z_{1}}^{z_{2}} \eta(\tau) d z\right) \frac{t_{b}}{l_{f}} .
$$

To eliminate $P_{f b}$, combine Eqs. 2.31 and 2.35, thus obtaining the following,

$$
q_{b}(\tau)=\frac{1}{C_{f b}(\tau)}\left(P-q_{b}(\tau) \frac{1}{S_{b}} \int_{z_{2}}^{z} \eta(\tau) d z\right)
$$

Solving for $q_{b}$ and including time dependency from Eq. 2.2, the blade flow rate is 


$$
q_{b}(\tau)=\frac{P}{C_{f b}(\tau)+\frac{1}{S_{b}} \int_{z_{1}}^{z} \eta(\tau) d z}=\phi \frac{d z}{d \tau}
$$

The solution of Eq. 2.38 yields the time for blade infiltration,

$$
\tau_{b}=\frac{\phi}{P} \int_{z_{2}}^{z_{3}}\left[\left(\frac{1}{S_{s}} \int_{0}^{z_{1}} \eta(\tau) d z \frac{l_{f}}{l_{s}}+\frac{1}{S_{f}} \int_{z_{1}}^{z_{2}} \eta(\tau) d z\right) \frac{t_{b}}{l_{f}}+\frac{1}{S_{b}} \int_{z_{2}}^{z} \eta(\tau) d z\right] d z
$$

\subsubsection{Permeability Computation}

The skin and flange permeabilities are those perpendicular to the fibers (Eq. 2.14). The blade permeability, however, is dependent on the stacking sequence and is computed by averaging the permeability of each layer, a method used by Fingerson $e t$ al. [23]. In the blade, permeability of $0^{\circ}$ plies is that perpendicular to the fiber direction (Eq. 2.14) since $0^{\circ}$ fibers run along the panel length direction and the flow is in the through-the-thickness direction. For $90^{\circ}$ plies, it is that in the fiber direction (Eq. 2.13) because $90^{\circ}$ plies have the fibers along the stiffener height direction. For $\pm 45^{\circ}$ plies, it is the average of these two permeabilities as given by Eq. 2.15 .

\subsection{Structural Analysis Model}

The planform of the stiffened panel is shown in Figure 2.11. The panel is subjected to a uniform end-shortening load $(F)$ in the $x$-direction and is simply supported along the four edges. This type of loading implies that the skin and stiffener elements deform together in multiple sinusoidal half-waves along the length and width of the panel. 


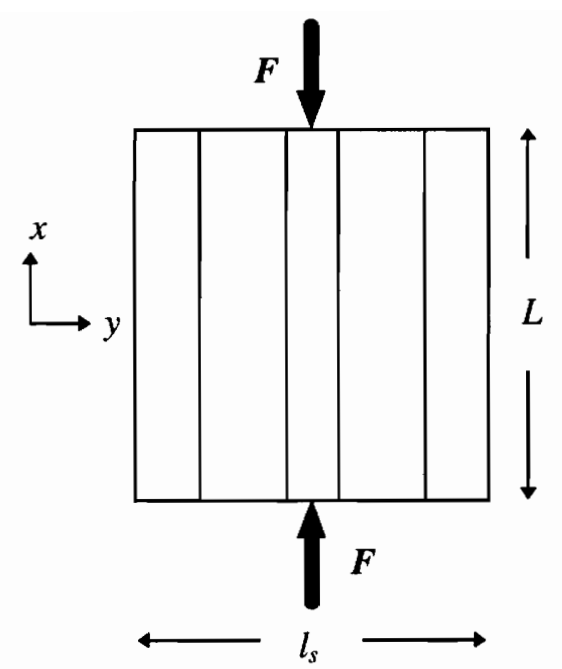

Figure 2.11 Panel Loading Condition

It is desired to determine the buckling response of the panel under this loading condition. As will be explained later, design variables that govern the manufacturing process also impact the structural behavior of the panel. One of the goals of this research is to explore the changes in the buckling response of the panel subject to changes in these design variables. As a result, a detailed buckling analysis would be prohibitively expensive at this point. Therefore, a simpler approach is taken.

One possible approach to the buckling analysis is to "smear" the engineering properties of the skin and stiffener. However, this method might eliminate some important aspects of the panel's buckling behavior, such as inter-stiffener buckling. To account for this, the panel is assumed to be composed of two separate structural elements as illustrated in Figure 2.12. Here, the skin structural element is the portion of the panel between the flanges of two stiffeners. The stiffener element consists of the blade, flange, and the portion of the skin beneath the flange. Under a uniform end shortening, the 
applied loading is distributed between the two elements proportional to their relative stiffnesses as follows,

$$
\begin{aligned}
F_{\text {skin }} & =\frac{(E A)_{s k i n}}{(E A)_{s i t f f}+(E A)_{s k i n}} F \\
F_{\text {stiff }} & =\frac{(E A)_{\text {stiff }}}{(E A)_{\text {stiff }}+(E A)_{s k i n}} F
\end{aligned}
$$

where $A$ represents the cross-sectional area and $E$ is Young's modulus.

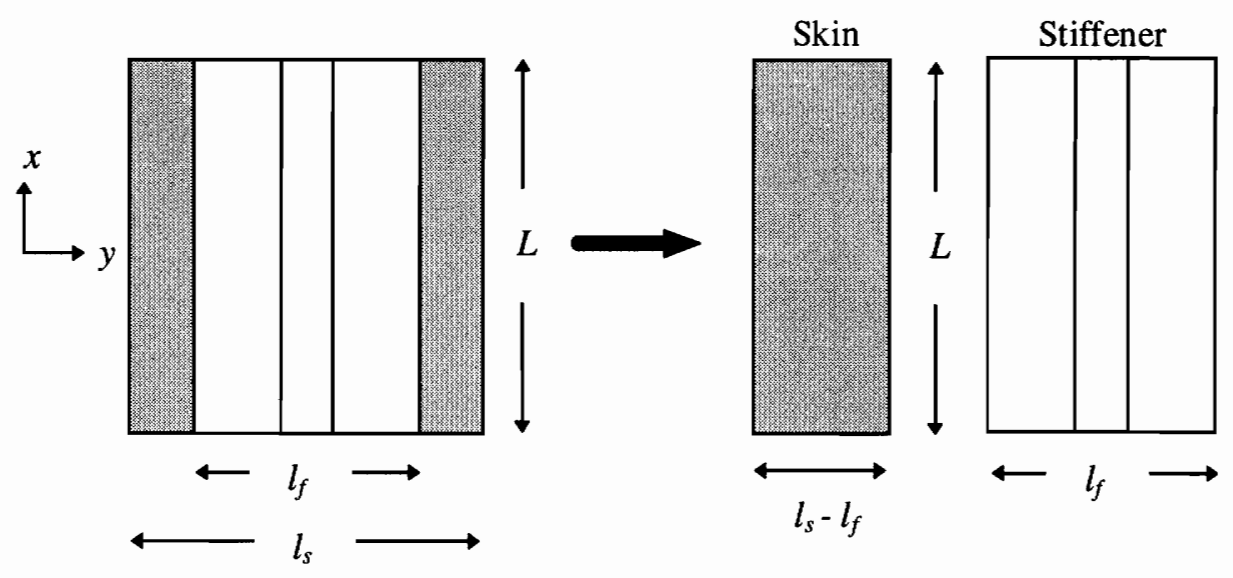

Figure 2.12 Skin and Stiffener Structural Elements

These forces are then compared to the critical loads that will cause each element to buckle; this will be discussed in Section 2.3.3. At this point, however, $F_{\text {skin }}$ and $F_{\text {stiff }}$ are unknown because their respective stiffnesses depend on the cross-sectional design variables, which are unknowns. In order to compute the engineering properties of the skin and stiffener, micromechanical principles and classical lamination theory is used. These properties will also allow the critical buckling loads to be determined. 


\subsubsection{Micromechanics and Material Properties}

In composites, both the fiber and resin materials have individual Young's moduli that describe their elastic behavior. For AS-4 graphite fibers, $E_{f}=207 \mathrm{GPa}$, and for Hercules 3501-6 epoxy resin, $E_{r}=3.14 \mathrm{GPa}$. Given the fiber volume fraction/porosity, the moduli of a composite material in the principal material directions can be computed. This is achieved using the law of mixtures [45],

$$
\begin{aligned}
& E_{1}=E_{f} v_{f}+E_{r} \phi \\
& \frac{1}{E_{2}}=\frac{v_{f}}{E_{f}}+\frac{\phi}{E_{r}}
\end{aligned}
$$

Recalling Eqs. 2.9 and 2.11, the fiber volume fraction and porosity are functions of the compaction pressure $(P)$. Therefore, this key manufacturing variable affects not only the resin infiltration of the panel, but also its material properties. Figure 2.13 illustrates the nature of this relationship between $E_{1}, E_{2}$, and $P$.

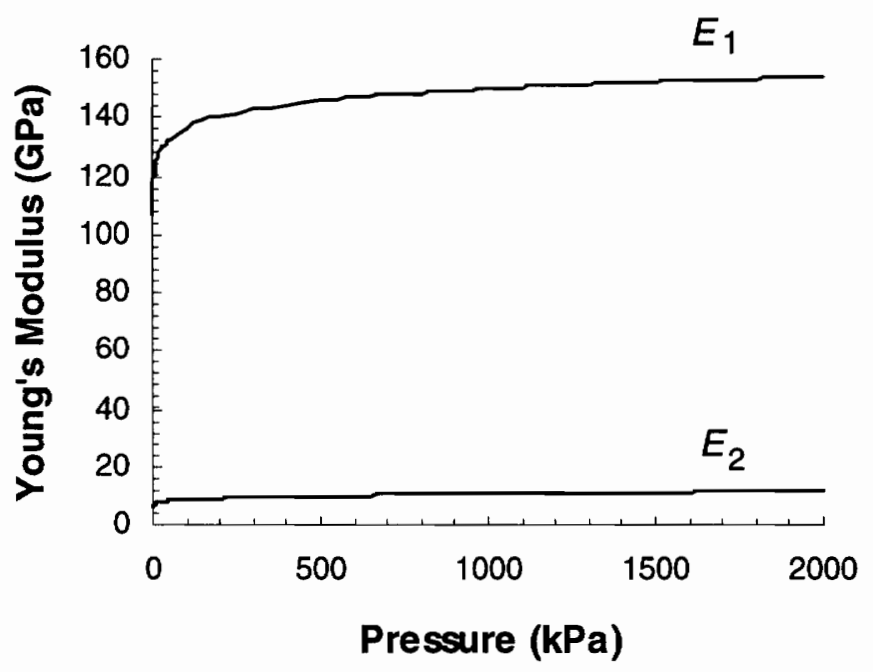

Figure 2.13 Principal Young's Moduli as a Function of Compaction Pressure 
The values of the other two material constants, the shear modulus $\left(G_{12}=7.19 \mathrm{GPa}\right)$ and the major Poisson's ratio $\left(v_{12}=0.3\right)$, are taken as those for AS-4/3501 prepregs. The minor Poisson's ratio is given by

$$
\mathrm{v}_{21}=\frac{E_{2}}{E_{1}} \mathrm{v}_{12} .
$$

Knowing the values of these four material constants $\left(E_{1}, E_{2}, G_{12}\right.$, and $\left.v_{12}\right)$, a reduced material stiffness matrix $\mathbf{Q}$ can be computed. $\mathbf{Q}$ relates in-plane stresses to strains in the principal material directions. The non-zero elements of this matrix are given below,

$$
\begin{aligned}
Q_{11} & =\frac{E_{1}}{1-v_{12} v_{21}} \\
Q_{12} & =\frac{v_{12} E_{2}}{1-v_{12} v_{21}} \\
Q_{22} & =\frac{E_{2}}{1-v_{12} v_{21}} \\
Q_{66} & =G_{12}
\end{aligned}
$$

From the reduced stiffnesses, a series of material parameters that are invariant with respect to fiber orientation can be calculated [46] as

$$
\begin{aligned}
& U_{1}=\frac{1}{8}\left(3 Q_{11}+3 Q_{22}+2 Q_{12}+4 Q_{66}\right) \\
& U_{2}=\frac{1}{2}\left(Q_{11}-Q_{22}\right) \\
& U_{3}=\frac{1}{8}\left(Q_{11}+Q_{22}-2 Q_{12}-4 Q_{66}\right) \\
& U_{4}=\frac{1}{8}\left(Q_{11}+Q_{22}+6 Q_{12}-4 Q_{66}\right) \\
& U_{5}=\frac{1}{8}\left(Q_{11}+Q_{22}-2 Q_{12}+4 Q_{66}\right)
\end{aligned}
$$


Since $E_{1}$ and $E_{2}$ are governed by the compaction pressure, $\mathbf{Q}$ and $U_{i}$ become functions of $P$ as well.

\subsubsection{Classical Lamination Theory}

Classical lamination theory (CLT) [46] is used to determine the effective engineering properties of a laminated composite. CLT assumes that all the plies are perfectly bonded together, with infinitely thin bondlines that cannot deform in shear. CLT gives both the in-plane and flexure properties of a laminate as functions of the material invariants $\left(U_{i}\right)$ and the fiber orientation angles. When the laminates are restricted to be symmetric, as in this work, there is no bending-extension coupling.

The extensional, or in-plane, stiffness matrix A relates the laminate's in-plane stress resultants to the strains at the mid-plane. The elements of $\mathbf{A}$ are

$$
\begin{aligned}
& A_{11}=U_{1} V_{0 A}+U_{2} V_{1 A}+U_{3} V_{3 A} \\
& A_{22}=U_{1} V_{0 A}-U_{2} V_{1 A}+U_{3} V_{3 A} \\
& A_{12}=U_{4} V_{0 A}-U_{3} V_{3 A} \\
& A_{66}=U_{5} V_{0 A}-U_{3} V_{3 A} \\
& A_{16}=U_{2} V_{2 A}+2 U_{3} V_{4 A} \\
& A_{26}=U_{2} V_{2 A}-2 U_{3} V_{4 A}
\end{aligned}
$$

The $V_{i A}$ terms are known as lamination parameters and are obtained by integrating functions of the fiber orientations $(\theta)$ through the thickness of the laminate, as shown in Eq. 2.46. Here, $h$ is the total thickness of the laminate, and $z$ is a generic through-thethickness coordinate. 


$$
\begin{aligned}
& V_{0 A}=h \\
& V_{1 A}=\int_{-h / 2}^{h / 2} \cos 2 \theta d z \\
& V_{2 A}=\int_{-h / 2}^{h / 2} \sin 2 \theta d z \\
& V_{3 A}=\int_{-h / 2}^{h / 2} \cos 4 \theta d z \\
& V_{4 A}=\int_{-h / 2}^{h / 2} \sin 4 \theta d z
\end{aligned}
$$

The flexural, or bending stiffness, matrix D relates the moment resultants to the curvature of the laminate. Its terms are

$$
\begin{aligned}
& D_{11}=U_{1} V_{0 D}+U_{2} V_{1 D}+U_{3} V_{3 D} \\
& D_{22}=U_{1} V_{0 D}-U_{2} V_{1 D}+U_{3} V_{3 D} \\
& D_{12}=U_{4} V_{0 D}-U_{3} V_{3 D} \\
& D_{66}=U_{5} V_{0 D}-U_{3} V_{3 D} \\
& D_{16}=U_{2} V_{2 D}+2 U_{3} V_{4 D} \\
& D_{26}=U_{2} V_{2 D}-2 U_{3} V_{4 D}
\end{aligned}
$$

Once again, $V_{i D}$ are lamination parameters obtained through integration,

$$
\begin{aligned}
& V_{0 D}=\frac{h^{3}}{12} \\
& V_{1 D}=\int_{-h / 2}^{h / 2} z^{2} \cos 2 \theta d z \\
& V_{2 D}=\int_{-h / 2}^{h / 2} z^{2} \sin 2 \theta d z \\
& V_{3 D}=\int_{-h / 2}^{h / 2} z^{2} \cos 4 \theta d z \\
& V_{4 D}=\int_{-h / 2}^{h / 2} z^{2} \sin 4 \theta d z
\end{aligned}
$$


The effective engineering properties of the laminate depend solely on the in-plane stiffnesses,

$$
\begin{aligned}
& E_{x}=\frac{A_{11} A_{22}-A_{12}^{2}}{h A_{22}} \\
& E_{y}=\frac{A_{11} A_{22}-A_{12}^{2}}{h A_{11}} \\
& \mathrm{v}_{x y}=\frac{A_{12}}{A_{22}} \\
& G_{x y}=\frac{A_{66}}{h}
\end{aligned}
$$

Because only uniaxial loading in the $x$-direction is being considered, only $E_{x}$ is needed and will henceforth be denoted by $E$. The effective Young's modulus for both the skin $\left(E_{\text {skin }}\right)$ and stiffener $\left(E_{\text {stiff }}\right)$ elements are computed in this fashion. The flexural stiffnesses will be used later in the buckling response of the skin in Section 2.3.3.1. It is important to point out here that the stiffness matrices, $\mathbf{A}$ and $\mathbf{D}$, as well as the engineering properties (Eq. 2.49) of the panel are all dependent on the compaction pressure.

\subsubsection{Buckling Response of the Panel}

Now that the properties of the skin and stiffener elements are known, their individual buckling responses can be computed. The results will yield the critical values of $F_{\text {skin }}$ and $F_{\text {stiff }}$ above which the panel elements will buckle.

\subsubsection{Skin Element}

The structural response of the skin element is computed as a laminated plate undergoing buckling. A simply supported laminated plate with dimensions $a$ and $b$ is 
shown in Figure 2.14. In terms of the dimensions shown in Figure 2.12, the skin element dimensions are $a=L$ and $b=l_{s}-l_{f}$. The plate is subjected to loads per unit length of $N_{x}$ and $N_{y}$. Here, $\lambda$ is an amplitude parameter.

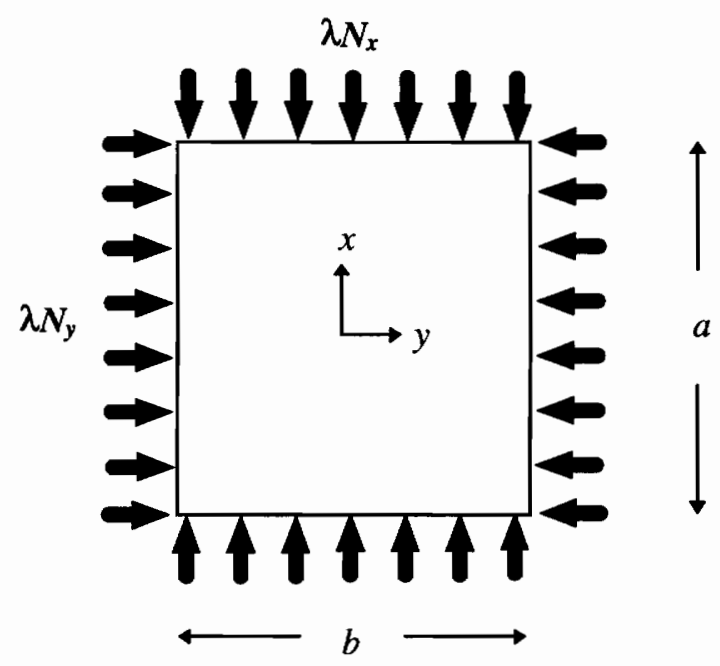

Figure 2.14 Plate Under Biaxial Compression

Under general biaxial compression, the laminate will buckle into $m$ and $n$ half waves in the $x$ - and $y$-directions, respectively, when the load amplitude reaches a critical value $\left(\lambda_{\text {buckle }}\right)$ given by [46]

$$
\lambda_{\text {buckle }}=\min _{m, n}\left\{\pi^{2}\left[\frac{D_{11}\left(\frac{m}{a}\right)^{4}+2\left(D_{12}+2 D_{66}\right)\left(\frac{m n}{a b}\right)^{2}+D_{22}\left(\frac{n}{b}\right)^{4}}{\left(\frac{m}{a}\right)^{2} N_{x}+\left(\frac{n}{b}\right)^{2} N_{y}}\right]\right\}
$$

The values of $m$ and $n$ are selected to minimize $\lambda_{\text {buckle }}$.

For the skin laminate at hand, which undergoes compression only in the $x$ direction, the critical load at which it will buckle can be obtained. Eq. 2.50 then becomes 


$$
F_{s k i n}^{c r i t}=\min _{m, n}\left\{\left(l_{s}-l_{f}\right)\left(\frac{\pi L}{m}\right)^{2}\left[D_{11}\left(\frac{m}{L}\right)^{4}+2\left(D_{12}+2 D_{66}\right)\left(\frac{m n}{L\left(l_{s}-l_{f}\right)}\right)^{2}+D_{22}\left(\frac{n}{l_{s}-l_{f}}\right)^{4}\right]\right\} \text {. }
$$

\subsubsection{Stiffener Element}

To compute its structural response, the stiffener element is treated as a column undergoing axial loading. The critical value of $F_{\text {stiff }}$ at which the column will buckle is given by Euler's formula,

$$
F_{\text {stiff }}^{\text {crit }}=\frac{\pi^{2}(E I)_{\text {stiff }}}{L^{2}}
$$

where $I_{\text {stiff }}$ is the moment of inertia of the stiffener cross-section, which depends on the stiffener geometry. To use Euler's formula, it must be assumed that $F_{\text {stiff }}$ is a perfectly aligned centric load.

In order to compute its moment of inertia, the stiffener is divided into three sections as shown in Figure 2.15.

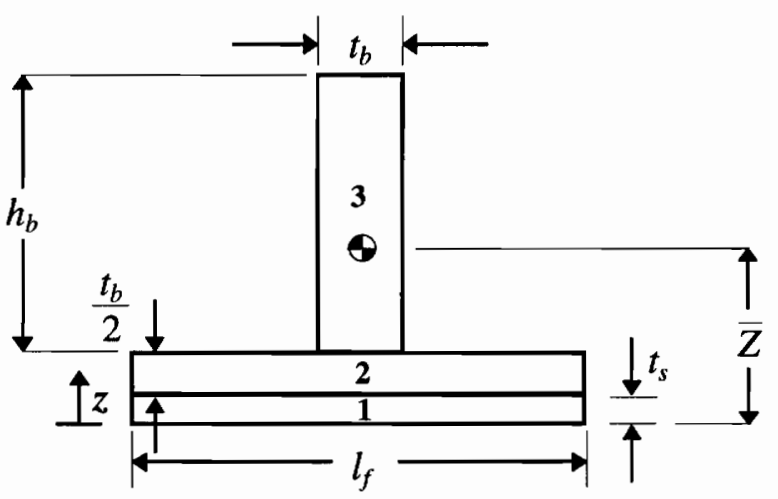

Figure 2.15 Stiffener Element Geometry

The stiffener moment of inertia is the sum of the moments of inertia of each section, 


$$
I_{\text {stiff }}=\sum_{i=1}^{3} I_{i},
$$

where $I_{i}$ are given by

$$
\begin{aligned}
& I_{1}=\frac{l_{f} t_{s}^{3}}{12}+A_{1}\left(\bar{z}_{1}-\bar{Z}\right)^{2} \\
& I_{2}=\frac{l_{f} t_{b}^{3}}{96}+A_{2}\left(\bar{z}_{2}-\bar{Z}\right)^{2} \\
& I_{3}=\frac{t_{b} h_{b}^{3}}{12}+A_{3}\left(\bar{z}_{3}-\bar{Z}\right)^{2}
\end{aligned}
$$

Here, $\bar{Z}$ is the $z$-coordinate centroid of the stiffener as illustrated in Figure 2.15,

$$
\bar{Z}=\frac{\sum_{i=1}^{3} A_{i} \bar{z}_{i}}{\sum_{i=1}^{3} A_{i}},
$$

$A_{i}$ are the cross-sectional areas, and $\bar{z}_{i}$ are the centroid locations of the individual sections as measured from the base of section 1. These parameters are given below,

$$
\begin{array}{ll}
A_{1}=l_{f} t_{s} & \bar{z}_{1}=\frac{t_{s}}{2} \\
A_{2}=\frac{l_{f} t_{b}}{2} & \bar{z}_{2}=t_{s}+\frac{t_{b}}{4} \\
A_{3}=t_{b} h_{b} & \bar{z}_{3}=t_{s}+\frac{t_{b}}{2}+\frac{h_{b}}{2}
\end{array}
$$

Substituting these terms into Eq. 2.55 , the stiffener centroid location becomes,

$$
\bar{Z}=\frac{h_{b}^{2} t_{b}+\left(h_{b}+\frac{l_{f}}{4}\right) t_{b}^{2}+\left(2 h_{b}+l_{f}\right) t_{b} t_{s}+l_{f} t_{s}^{2}}{2 h_{b} t_{b}+l_{f} t_{b}+2 l_{f} t_{s}} .
$$


With this expression and Eqs. 2.54 and 2.56, the moment of inertia of the stiffener can be computed.

The stiffener modulus $\left(E_{s t i f f}\right)$ is computed by classical lamination theory as was discussed in Section 2.3.2. Because $F_{\text {stiff }}$ acts through the centroid, the laminate used in the calculation combines the stacking sequences of the skin, flange, and blade laminates. For example, if $\left[0^{\circ}{ }_{80}\right],\left[0^{\circ}{ }_{40}\right]$, and $\left[ \pm 45^{\circ}{ }_{20}\right]$ are the stacking sequences of the skin, flange, and blade, respectively, the "stiffener laminate" is given by $\left[0^{\circ}{ }_{120} \mathrm{~d} \pm 45^{\circ}{ }_{20}\right]$. 


\section{Optimization Problem}

\subsection{Formulation}

An optimization problem seeks to maximize or minimize a measure of a design's performance, its objective function. The optimizer does this by varying key parameters (design variables) of the design until no further improvement in the objective function can be made. Constraints are also placed on the design variables to eliminate unacceptable (infeasible) designs. This type of parameter optimization is often referred to as mathematical programming. The combined structural and manufacturing optimization problem for the stiffened panel is described in the subsections that follow.

\subsubsection{Mathematical Programming}

The standard formulation of a constrained optimization problem is given below,

$$
\begin{array}{ll}
\text { minimize } & \psi(\mathbf{x}) \\
\text { subject to } & \left\{\begin{array}{l}
g_{i}(\mathbf{x}) \leq 0, i=1, \ldots, n_{\text {ineq }} \\
h_{j}(\mathbf{x})=0, j=1, \ldots, n_{\text {eq }}
\end{array}\right.
\end{array}
$$

Here, $\mathbf{x}$ is a vector of the design variables, $\psi$ is the objective function, $g_{i}$ are the inequality constraints, and $h_{j}$ are the equality constraints. There are a total of $n_{\text {ineq }}$ and $n_{e q}$ inequality and equality constraints, respectively. Note that if the objective were to maximize $\psi$, this could be achieved by minimizing $-\psi$. 
The design variables (x) come in two varieties: continuous and discrete. Continuous variables are those with infinite precision that are allowed to take on any value in the specified domain. Discrete variables, on the other hand, are only allowed to take on particular values in the domain. Integers are an example of discrete variables.

The statement in Eq. 3.1 is that of a single-objective optimization problem since there is only one objective function. Frequently, as will be the case in this work, there may be several objectives to be optimized $\left(\psi_{1}, \psi_{2}\right.$, etc. $)$. This type of problem is referred to as a multicriteria optimization problem.

\subsubsection{Design Variables}

There are three sets of design variables to be optimized: geometric, material, and manufacturing. The variables are selected as those that impact both the manufacturing and structural response of the panel. The geometric variables define the cross-section of the panel as was shown in Figure 2.7. These are the skin and blade thicknesses, the skin and flange widths, and the blade height. Note that the panel length $(L)$ remains constant because it has no impact on the one-dimensional manufacturing analysis.

Material variables come in the form of the ply orientation angles (i.e., stacking sequences) of the skin and blade laminates. Both of the laminates are taken to be balanced and symmetric. Because the flange is an extension of one-half of the blade laminate, as was described in Section 2.2.1, its stacking sequence is also half that of the blade, and thus, is not symmetric. To reduce the number of material variables, the plies are further arranged into "stacks," consisting of four plies of the same orientation (a $45^{\circ}$ stack 
consists of two $+45^{\circ}$ plies and two $-45^{\circ}$ plies to maintain the balanced condition). The three possible stacks are $0^{\circ}{ }_{4}, \pm 45^{\circ}$, and $90^{\circ}$. Thus, for a $N$-ply symmetric laminate in this work, only $\frac{N}{8}$ stacks have to be considered in the optimization. Previous research [3-6, 8-10] has employed two-ply stacks for a total of $\frac{N}{4}$ for balanced and symmetric laminates. However, in order to decrease the total number of design variables, the fourply stacks are preferred.

The material variables govern the thicknesses of the skin and blade. For $N_{s}$ and $N_{b}$ plies in the skin and blade laminates, respectively, the thicknesses are

$$
\begin{aligned}
& t_{s}=N_{s} t_{p l y} \\
& t_{b}=N_{b} t_{p l y}
\end{aligned}
$$

The material variables also affect both the structural and manufacturing responses of a panel design. The in-plane properties are dependent on the total numbers of each ply orientation, while the stacking sequence itself governs the flexural properties. In addition, the stacking sequence of the blade laminate also determines its permeability as was discussed in Section 2.2.3.

The manufacturing variables are the compaction pressure and the cure temperature. As was discussed in Section 2.3.1, pressure not only affects the resin infiltration time, but also the panel's structural response by changing its material properties. Although the temperature has no direct impact on structural performance, it plays too large a role in the manufacturing analysis to remain a constant. Variations in 
cure temperature may lead to premature gelling or curing of the resin before the infiltration process is completed.

The optimization problem includes both continuous and discrete design variables. The geometric and manufacturing variables are continuous, but the stacking sequence optimization is a discrete problem since the ply orientations are limited to three choices. The optimization procedure used must therefore account for this. The optimization problem is described below.

\subsubsection{Objective Function}

The optimization problem at hand is a multicriteria one. There are two objectives in this problem, one structural and one manufacturing. The structural objective is to minimize the mass of the panel,

$$
M=L A\left(v_{f} \rho_{f}+\phi \rho_{r}\right)
$$

where $L$ is the panel length, $A$ is the total cross-sectional area of the panel, $v_{f}$ is the fiber volume fraction, $\phi$ is the porous volume fraction occupied by the infiltrated resin, and $\rho_{f}$ $\left(1.79 \times 10^{3} \mathrm{~kg} / \mathrm{m}^{3}\right)$ and $\rho_{r}\left(1.26 \times 10^{3} \mathrm{~kg} / \mathrm{m}^{3}\right)$ are the fiber and resin densities, respectively. On the manufacturing side, the goal is to minimize the resin infiltration time, the sum of the individual times for each panel section (Eqs. 2.20, 2.29, and 2.39),

$$
\tau=\tau_{s}+\tau_{f}+\tau_{b}
$$

To simplify the multicriteria problem, a single composite objective function $(\psi)$ is created that incorporates both Eq. 3.3 and 3.4. Thus, the optimization problem becomes 


$$
\min \psi=\beta_{1} M+\beta_{2} \tau,
$$

where $\beta_{1}$ and $\beta_{2}$ are weighting parameters that govern the relative importance and normalize the magnitudes of the individual objectives.

\subsubsection{Constraints}

Constraints must also be placed on both the structural and manufacturing analyses. The structural constraints are derived from the buckling analyses of the skin and stiffener element of the panel. The actual loading in each element (Eq. 2.40) cannot exceed its critical buckling value (Eqs. 2.51 and 2.52). These constraints are expressed mathematically as follows,

$$
\begin{aligned}
& g_{1}=\frac{F_{\text {skin }}}{F_{\text {skin }}^{\text {crit }}}-1=r_{\text {skin }}-1 \leq 0 \\
& g_{2}=\frac{F_{\text {stiff }}}{F_{\text {stitf }}^{\text {crit }}}-1=r_{\text {stiff }}-1 \leq 0
\end{aligned}
$$

During the resin infiltration process, it is desired that the preform of the panel be completely filled with resin prior to the resin gelation, as was discussed in Section 2.1.3. This is the basis of the manufacturing constraint,

$$
g_{3}=0.99-\Lambda \leq 0,
$$

where $\Lambda$ is the percentage of the preform filled with resin. The constraint is relaxed to allow $99 \%$ and above to be considered completely filled. If the preform is not completely filled, $\Lambda$ represents the percent filled when the resin has gelled, and the constraint is violated. 


\subsection{Genetic Algorithms}

A genetic algorithm (GA) [47-49] is a probabilistic optimization method that works on a population of designs by mimicking the processes of genetic evolution. The two fundamental operators in a GA are crossover, a reproductive operator to create new individuals (i.e., designs), and mutation, an operator that helps preserve diversity in the population and prevent it from stagnating. A process based on natural selection determines which members of the population survive to reproduce.

GA's have several advantages over other optimization procedures. They do not require any gradient or derivative information and are also insensitive to the complexity of the objective function. For this work, the strongest reason for selecting the GA is its ability to deal with discrete design variables. A typical drawback of the GA is its high computational cost. GA populations require large numbers of objective function evaluations, typically ranging in the thousands, or even millions. However, the analysis models used in this work are not computationally prohibitive. Thus, the advantages to using the GA outweigh this disadvantage.

\subsubsection{Design Encoding}

In order to be used with the GA, each design must be encoded into a finite string of integer digits, called alleles. This string serves as the design's "chromosome." This chromosome is typically expressed in binary, but not all problems lend themselves well to this type of representation. As will be explained in the subsequent sections, a ternary 
encoding is preferred in the present work. Furthermore, because the optimization problem involves both discrete and continuous design variables, the chromosome is broken down into substrings for each design variable. These substrings are encoded separately but use a common set of ternary alleles. Each allele in the chromosome is a variable that the GA manipulates in order to obtain an optimal design.

\subsubsection{Discrete Variables}

The discrete design variable substrings describe the stacking sequences of the skin and blade laminates. Following the work of Le Riche and Haftka [8-9], each stack in the laminate is assigned an allele of " $1, "$ " 2 ," or " 3, , corresponding to the three ply orientations available, $0^{\circ}{ }_{4}, \pm 45^{\circ}$, and $90^{\circ}$, respectively. The skin laminates, which consist of 40 plies, require five alleles for its encoding, while the 80-ply blade laminates require 10 alleles. The laminates are encoded "outside-in," with the first element in the string corresponding to the outermost stack and the last element in the string corresponding to stack closest to the laminate plane of symmetry. The encodings for the skin and blade laminates are then spliced together to form the discrete variable substring. For example, the substring ( $32213: 1123322212$ ) describes a skin laminate $\left[90^{\circ}{ }_{4} / \pm 45^{\circ}{ }_{4} / 0^{\circ}{ }_{4} / 90^{\circ}{ }_{4}\right]_{s}$ and a blade laminate $\left[0^{\circ}{ }_{8} / \pm 45^{\circ} 2 / 90^{\circ}{ }_{8} / \pm 45^{\circ}{ }_{6} / 0^{\circ}{ }_{4} / \pm 45^{\circ}{ }_{2}\right]_{s}$. $\Theta_{s}$ and $\Theta_{b}$ will be used to denote the substring encodings of the skin and blade stacking sequences, respectively.

\subsubsection{Continuous Design Variables}

In order to optimize continuous variables with a GA, they must be discretized in order to be encoded. Because of the ternary allele set used in the stacking sequence 
encoding, the continuous variables become functions of integers expressed in base-three. These base-three integers $\left(\Omega_{N}\right)$ can be represented as

$$
\Omega_{N}=\sum_{i=1}^{N}\left(\omega_{i}-1\right) 3^{i-1},
$$

where $\omega_{i}$ is the value of the $i^{\text {th }}$ allele $(1,2$, or 3$)$, and $N$ is the number of alleles used in the encoding. For example for the substring (13212), $N=5$; thus, $\Omega_{5}=96$.

For the blade height, five alleles are used in the encoding. The value of $h_{b}$, in meters, is computed as

$$
h_{b}=0.0005\left(1+\Omega_{5}\right) \text {. }
$$

This formulation allows this parameter to vary between $0.0005 \mathrm{~m}$ and $0.1215 \mathrm{~m}$ in 0.0005 $\mathrm{m}$ increments, which is adequate for the problem at hand. Note that for computational reasons, $h_{b}$ is not allowed to take on zero values. As an example, the substring (1 2132$)$ denotes a blade height of $0.0690 \mathrm{~m}$.

Five alleles are also used in the encoding of the skin width. Unlike the blade height, there is a restriction to the width of the skin in that it cannot be less than the thickness of the blade. Taking this into account, the value of $l_{s}$, in meters, is computed as

$$
l_{s}=t_{b}+0.001 \Omega_{5} .
$$

Since the range of $l_{s}$ is dependent on $t_{b}$, it can not be explicitly stated. Nevertheless, the step size is $0.001 \mathrm{~m}$. 
The width of the flange is expressed as a percentage of the skin width in order to prevent the flange from numerically becoming wider than the skin during the optimization. Its value is given by

$$
l_{f}=l_{s}\left(0.01+0.012375 \Omega_{4}\right) .
$$

Here, only four alleles are used to provide $l_{f}$ with a range of $0.01 l_{s}$ to $l_{s}$ in $0.012375 l_{s}$ increments. For example, (3212) would represent $0.406 l_{s}$. The value of 0.012375 comes from dividing 0.99 (the skin width percentage range over which the flange can vary, $1-0.01$ ) by the maximum allowable value of $\Omega_{4}, 80$. As with the skin width, the blade thickness cannot exceed the flange width. Therefore, if $l_{f}<t_{b}, l_{f}$ is assigned the value of $t_{b}$.

The cure temperature in ${ }^{\circ} \mathrm{C}$ is also encoded as a substring of four alleles and is calculated as

$$
T=100+\Omega_{4} .
$$

The range of $T$ is therefore $100^{\circ} \mathrm{C}$ to $180^{\circ} \mathrm{C}$ in $1^{\circ}$ increments. The minimum value of $T$ is selected arbitrarily based on the cure behavior of the resin. The maximum value of $180^{\circ} \mathrm{C}$ using four alleles is also sufficient. Using this scheme, for example, a temperature of $119^{\circ} \mathrm{C}$ is encoded as $(2131)$.

The last continuous design variable to be encoded is the compaction pressure,

$$
P=5\left(1+\Omega_{5}\right) .
$$

Five alleles yield a pressure range of $5 \mathrm{kPa}$ to $1,215 \mathrm{kPa}$ in $5 \mathrm{kPa}$ increments. Thus, for example, the substring (31323) denotes a pressure of $1,050 \mathrm{kPa}$. Using four alleles 
would allow the pressure to reach a maximum of only $405 \mathrm{kPa}$, which is low for the RFI process.

\subsubsection{GA Operators}

Figure 3.1 is a flow chart of the basic GA. The GA begins with an initial population of $\Pi$ designs randomly generated. The objective function value for each individual design in the population is evaluated to assess its performance. The individuals are then ranked according to their objective function values and assigned a fitness value ( $\Phi=0$ for the worst design, and $\Phi=\Pi-1$ for the best). New designs are created through the processes of selection, crossover, and mutation discussed below. The optimization procedure is repeated until a specified number of generations has passed or after a specified number of generations without any improvement in the objective function value of the best design is reached.

\subsubsection{Selection}

Using a "roulette wheel" strategy suggested by Davis [48], two parents are selected as to give the most fit (here, those with low objective function values) designs a better chance of reproducing. A graphical representation of a roulette wheel for a population of four designs is shown in Figure 3.2. Each design is given a portion of the wheel proportional to its fitness. When the wheel is "spun," designs that occupy a larger portion of the wheel (i.e., those with high fitness values) are more likely to be selected. 


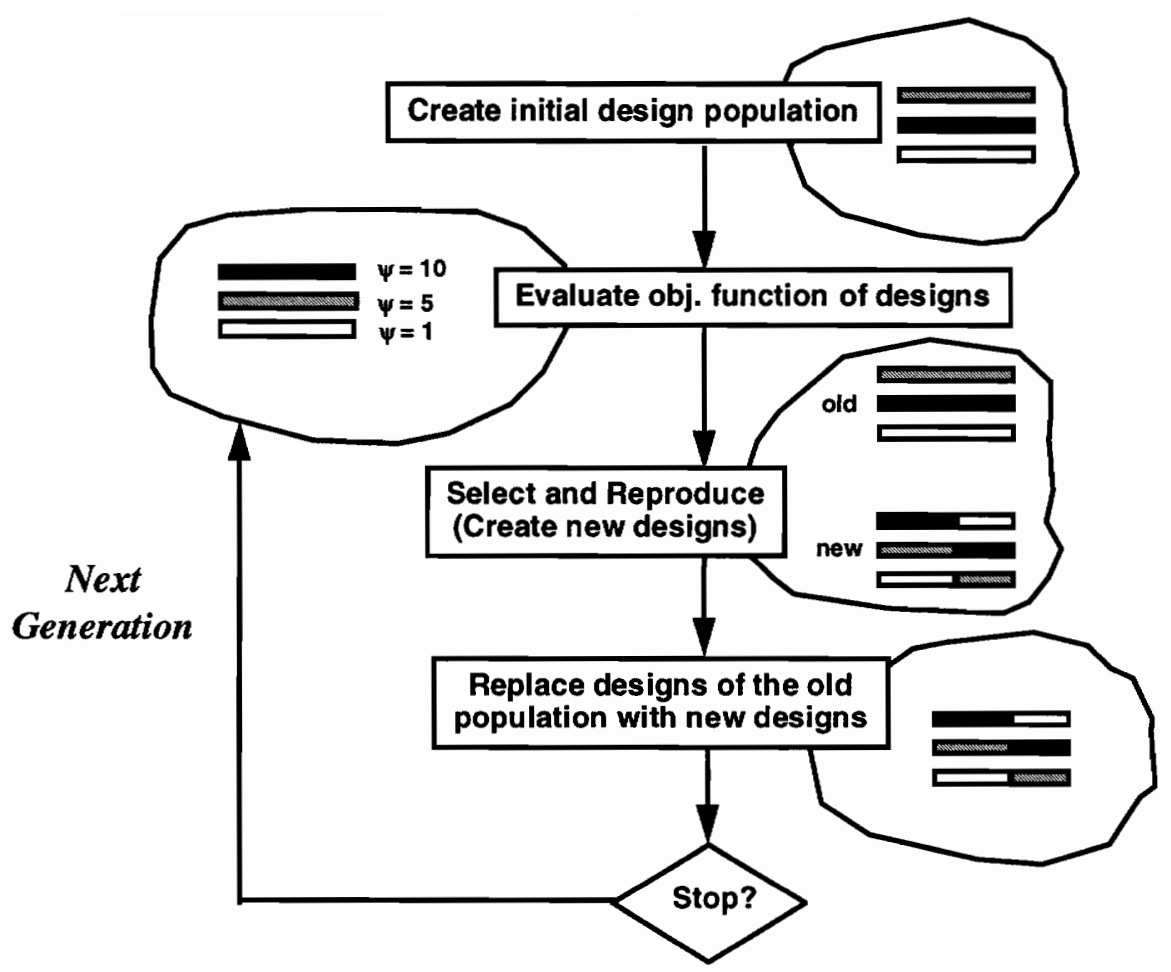

Figure 3.1 Genetic Algorithm Flowchart

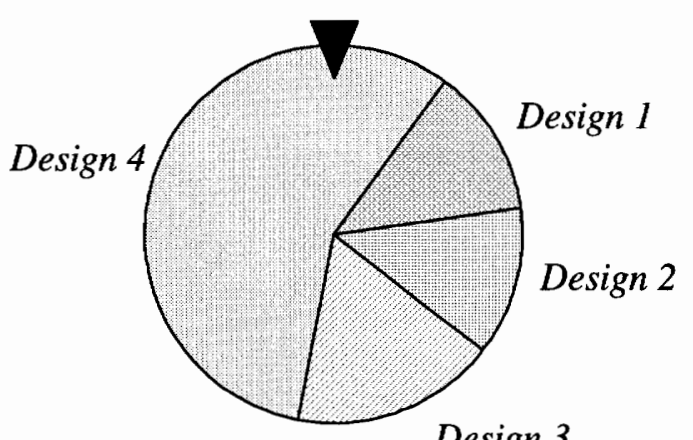

Design 3

Figure 3.2 Roulette Wheel for Parent Selection

Mathematically, the roulette wheel is handled as follows. The fitness values of the designs are incrementally summed as shown in Eq. 3.14, 


$$
\begin{gathered}
s_{1}=\Phi_{1} \\
s_{2}=\sum_{i=1}^{2} \Phi_{i} \\
\vdots \\
s_{\Pi}=\sum_{i=1}^{\Pi} \Phi_{i}
\end{gathered}
$$

A random number $(R)$ between zero and $s_{\Pi}$ is then generated. If $s_{i}<R \leq s_{i+1}(i=1, \ldots$, $\Pi-1)$, design $i+1$ is selected. The process is then repeated to select the second parent. Selection allows the parent designs with the most desirable characteristics to be copied into the next generation of designs by the crossover process.

\subsubsection{Crossover}

As previously described, crossover is the reproductive operator of the GA. Two selected parents "mate" to create a new child design. Traditionally, the crossover operation is performed by randomly selecting a break point between two alleles along the length of the chromosome string. Two children are then created by swapping the righthand substring portions of the two parents' chromosomes as shown in Figure 3.3.

Parent Designs

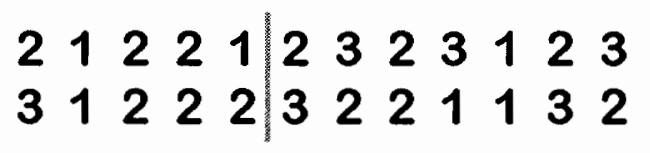

Resulting Children

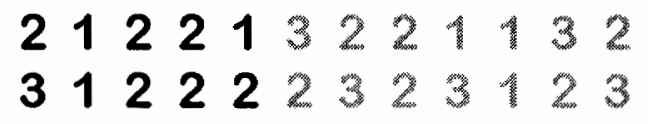

Figure 3.3 Traditional Crossover Example 
In this work, a two-point crossover [8] is used, whereby two break points along the string are chosen at random, and two children are created by swapping the middle substrings of the parents' chromosomes. Figure 3.4 illustrates two-point crossover.

Parent Designs

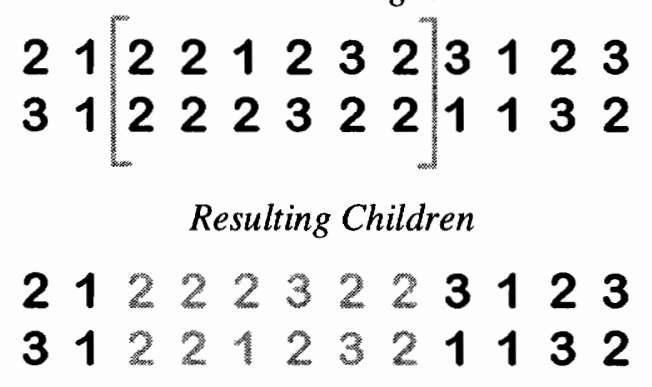

Figure 3.4 Two-Point Crossover Example

Only one of the resulting children is kept; the choice is again random. The selection and crossover processes repeat until enough children are created to replace all but the best member of the old population. This approach to the GA is called an "elitist plan" since the best design is always carried into the next generation.

\subsubsection{Mutation}

Just as random mutations occur in genetic evolution, they are also incorporated into the GA. Given the newly created children designs from the crossover process, a mutation operation is performed with a given probability $\left(p_{m u t}\right)$. This is done by randomly switching an allele $(1,2$, or 3$)$ to one of the other two choices available. Mathematically, each allele in a child design is assigned a random number $\left(0 \leq R_{i} \leq 1\right)$. If $R_{i}<p_{m u t}$, that allele is changed. Figure 3.5 illustrates the mutation processes with $p_{m u t}=0.001$. The numbers below the alleles of the original design represent the values of $R_{i}$. 
Original Design

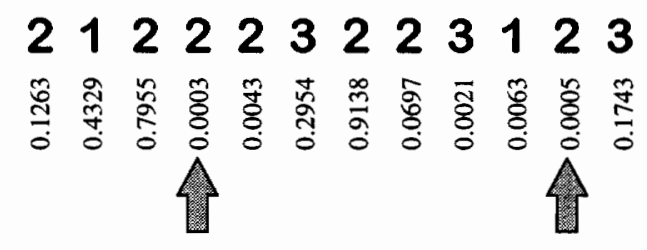

Mutated Design

\section{3}

Figure 3.5 Mutation Example

\subsubsection{Permutation}

Another operator created for laminate design by Le Riche and Haftka [8-9] is permutation. As with mutation, permutation is applied with a certain probability $\left(p_{p e r}\right)$ to the children designs created during crossover. Two random locations along the chromosome are chosen, and the substring bounded by these points is then inverted (i.e., its allele order is reversed). An example of permutation is illustrated in Figure 3.6.

Original Design

\section{$2122\left[\begin{array}{llllll}1 & 2 & 3 & 2 & 3 & 2\end{array}\right] 23$}

Permutated Design

\section{3}

\section{Figure 3.6 Permutation Example}

In stacking sequence optimization, permutation is beneficial because it changes the flexural properties of a laminate while preserving its in-plane characteristics. 


\subsubsection{Optimization Problem for GA}

The GA is an unconstrained optimization procedure. Therefore, in order to incorporate the necessary constraints, a penalty function approach is taken. Penalty functions augment the objective function and are used in a minimization problem to increase the value of the objective function when constraint violations are encountered.

Because there are two separate objectives in this problem, each is penalized separately. The augmented panel mass $\left(M^{*}\right)$ is given by the following expression,

$$
M^{*}=\left\{\begin{array}{l}
\beta_{1} M+\chi_{1} \max \left(g_{1}, g_{2}\right), \text { if } g_{1}>0 \text { or } g_{2}>0 \\
\beta_{1} M+\varepsilon_{1} \min \left(g_{1}, g_{2}\right), \text { if } g_{1} \leq 0 \text { and } g_{2} \leq 0
\end{array}\right.
$$

Here, $\chi_{1}$ is the parameter that penalizes $\beta_{1} M$ for constraint violations, and $\varepsilon_{1}$ is a bonus parameter. Both of these quantities are positive. The bonus parameter is used to give a slight advantage to those designs that with large constraint margins while satisfying both of the structural constraints ( $g_{1}$ and $g_{2}$ ) by reducing their objective function values compared to the designs that just satisfy the constraints with no margin. Note that the sign in the second expression of Eq. 3.15 is positive because both $g_{1}$ and $g_{2}$ would be negative in this situation. The bonus also distinguishes between designs of the same mass, but with different degrees of constraint satisfaction. This means that two designs with identical geometric variables but different stacking sequences will have different buckling responses, as mentioned in Section 3.1.2, and that the design with a better buckling margin is given a larger bonus compared to the other one.

The augmented infiltration time $\left(\tau^{*}\right)$ is 


$$
\tau^{*}=\beta_{2} \tau+\chi_{2} \max \left(g_{3}, 0\right) .
$$

Again, $\chi_{2}$ is a penalty parameter for designs not satisfying the fill constraint $\left(g_{3}\right)$. No bonus is used because two designs with the same infiltration time will have the same percentage filled. Using the two penalized objectives, a new composite objective function ( $\Psi$ ) can be formed. The unconstrained optimization problem for use with the GA then becomes

$$
\min \Psi=M^{*}+\tau^{*} .
$$




\section{RESUltS AND DiSCUSSION}

Now that the structural and manufacturing analysis models have been developed, and the optimization problem has been formulated, results will be presented. These results will illustrate the nature of the structural and manufacturing responses of blade-stiffened panel designs and how changing the design variables will impact these responses. To illustrate the motivation behind the multidisciplinary approach to the problem, this chapter begins with the discussion of optimal panel designs from a previous study.

Table 4.1 presents the 10 best designs obtained by Nagendra et al. [4] from a study of the weight minimization of a blade-stiffened panel with buckling constraints. The design variables used in that study were the blade height $\left(h_{b}\right)$ and the stacking sequences of the skin and blade laminates whose encodings are represented in the table by $\Theta_{s}$ and $\Theta_{b}$, respectively. The thicknesses of the laminates were also allowed to vary in stacks of four plies with the total number of plies being $N_{s}$ and $N_{b}$ for the skin and blade, respectively. The values of skin width $\left(l_{s}=20.30 \mathrm{~cm}\right)$ and flange width $\left(l_{f}=6.10 \mathrm{~cm}\right)$ were fixed, and the panels were designed to carry an axial load of $3.50 \mathrm{MN} / \mathrm{m}$ and a shear load of 0.876 $\mathrm{MN} / \mathrm{m}$. The designs in Table 4.1, listed in order of increasing panel mass as obtained by Nagendra et al., were then analyzed by the one-dimensional RFI manufacturing model to compute the time required to fully infiltrate the panels with these geometries. Because the weight minimization problem in [4] did not require any manufacturing-related variables, values for the applied pressure and temperature must be chosen to perform the 
manufacturing analysis. These values were arbitrarily selected to be $700 \mathrm{kPa}$ and $120^{\circ} \mathrm{C}$, respectively. Included in Table 4.1 are the resin infiltration times $(\tau)$ and the ranking of the designs in terms of this measure of manufacturing efficiency. The masses $(M)$ given in the table are for panels composed of four stiffener elements, whereas the infiltration time is computed for the unit cell since the manufacturing analysis is one-dimensional.

Table 4.1 Manufacturing Analyses of Minimum Weight Designs from Ref. [4]

\begin{tabular}{cccccccc}
\hline $\begin{array}{c}\boldsymbol{h}_{\boldsymbol{b}} \\
(\mathbf{c m})\end{array}$ & $\boldsymbol{N}_{\boldsymbol{s}}$ & \multicolumn{1}{c}{$\Theta_{s}$} & $N_{\boldsymbol{b}}$ & \multicolumn{1}{c}{$\Theta_{b}$} & $\begin{array}{c}\boldsymbol{M} \\
(\mathbf{k g})\end{array}$ & \multicolumn{1}{c}{$\begin{array}{c}\boldsymbol{c}(\mathbf{s}) \\
\text { Rank }\end{array}$} \\
\hline 8.03 & 32 & 23323222 & 76 & 222112121212113113 & 10.95 & 1,181 & 4 \\
7.72 & 32 & 23232222 & 72 & 221121121211313121 & 10.96 & 1,012 & 2 \\
8.09 & 32 & 23322222 & 76 & 2222112121131121131 & 10.98 & 3,225 & 9 \\
7.75 & 36 & 233232222 & 72 & 221211212112113121 & 10.98 & 1,249 & 5 \\
7.89 & 36 & 232332222 & 72 & 222221121131121131 & 11.04 & 1,275 & 6 \\
8.06 & 36 & 233222222 & 72 & 222221121131121131 & 11.12 & 1,474 & 7 \\
7.72 & 36 & 233232222 & 72 & 222121121121131131 & 11.15 & 1,174 & 3 \\
7.74 & 32 & 23222222 & 80 & 22211212121121121131 & 11.16 & gelled & 10 \\
7.80 & 40 & 2332223222 & 68 & 22211211311212112 & 11.16 & 981 & 1 \\
7.74 & 36 & 233222222 & 76 & 2211211212112113112 & 11.17 & 1,712 & 8 \\
\hline
\end{tabular}

From this table, it can be seen that the lightest design only ranks fourth in terms of its infiltration time while the ninth-best structural design ranks first, and therefore, is the design that can be manufactured the fastest. Note that the weight difference between the best and tenth-best designs is only about $2 \%$, whereas there is more than a factor of three difference between the lowest and highest infiltration times. Also note that the resin gels before infiltration is complete in the design ranked tenth. Although no direct relationship between the structural and manufacturing objectives can be made from the data, Table 4.1 shows that these objectives differ substantially enough to warrant a multiobjective approach to the optimization problem. The results also suggest that it is possible to 
achieve substantial gains in manufacturing time without much of a loss in structural performance.

\subsection{Parametric Studies}

It is desired to observe the effects of the geometric and manufacturing design variables on both the manufacturing and structural analyses. To achieve this, a baseline design is selected partially based on the results of Nagendra et al. [4] and analyzed using the structures and manufacturing models developed in Chapter 2. The values of the baseline design variables, as well as the values of the resulting structural and manufacturing objectives and constraints are presented in Table 4.2. Note that the skin and blade thicknesses are not treated as design variables per se. Recall from Eq. 3.2 that $t_{s}$ and $t_{b}$ are functions of the ply thickness and the total number of plies. The ply thickness is governed by the compaction pressure, which controls the fiber volume fraction as was shown in Eqs. 2.11 and 2.12. Therefore, since the numbers of plies in the laminates are kept fixed in this work, $t_{s}$ and $t_{b}$ depend solely on the pressure and are not varied independently.

From these results, it can be seen that the resin infiltration time for the baseline design is quite high. In addition, both structural and manufacturing constraints are violated. The violation in the skin buckling constraint (recall from Chapter 2 that the skin and stiffener are treated as separate elements) can be attributed to the fact that the structural analysis model used in this work is more simplistic than that used by Nagendra 
et al. The skin buckling ratio (actual loading to critical buckling load) from Eq. 3.6 for the baseline design is $95.8 \%$ above its maximum allowable value, and the resin has gelled when only $93.8 \%$ of the preform is filled. In the subsections that follow, the skin width, flange width, blade height, cure temperature, and compaction pressure will each be varied individually while maintaining all others design variables fixed.

Table 4.2 Baseline Design

\begin{tabular}{ll}
\hline Geometric Variables & \\
\hline Skin Width $\left(l_{s}\right)$ & $20.30 \mathrm{~cm}$ \\
Flange Width $\left(l_{f}\right)$ & $6.10 \mathrm{~cm}$ \\
Blade Height $\left(h_{b}\right)$ & $7.60 \mathrm{~cm}$ \\
Skin Thickness $\left(t_{s}\right)$ & $0.479 \mathrm{~cm}$ \\
Blade Thickness $\left(t_{b}\right)$ & $0.957 \mathrm{~cm}$ \\
Material Variables & \\
\hline Skin Stacking Sequence $\left(\Theta_{s}\right)$ & 12312 \\
Blade Stacking Sequence $\left(\Theta_{b}\right)$ & 1231231231 \\
Manufacturing Variables & \\
\hline Compaction Pressure $(P)$ & $700 \mathrm{kPa}$ \\
Cure Temperature $(T)$ & $120^{\circ} \mathrm{C}$ \\
Structural Analysis & \\
\hline Applied Loading $(F)$ & $800 \mathrm{kN}$ \\
Panel Mass $(M)$ & $2.48 \mathrm{~kg}$ (unit cell) \\
& $9.92 \mathrm{~kg}$ (mult. stiff.) \\
Stiffener Buckling Ratio $\left(r_{s t i f f}\right)$ & 0.434 \\
Skin Buckling Ratio $\left(r_{s k i n}\right)$ & 1.958 \\
Manufacturing Analysis & \\
\hline Resin Infiltration Time $(\tau)$ & $2,985 \mathrm{~s}$ \\
Percentage Filled $(\Lambda)$ & 0.938 \\
\hline
\end{tabular}

Notice that two values for the panel mass are listed in Table 4.2. The first quantity is the mass of a unit stiffener cell as given by Eq. 3.3. However, as will be explained in Section 4.2.2.1, the behavior of the structural optimization warrants a new panel mass 
formulation. Recall from Figure 2.8 that a panel with a fixed overall width of $W$ (here, $81.2 \mathrm{~cm}$ ) will consist of $\frac{W}{l_{s}}$ stiffeners. The expression for the panel mass then becomes

$$
M=L A \frac{W}{l_{s}}\left(v_{f} \rho_{f}+\phi \rho_{r}\right)
$$

Although the baseline design consists of exactly four stiffener elements, it is inconsequential for this work if there are a non-integer number of stiffeners. Therefore, the second mass quantity in Table 4.2 is that given by Eq. 4.1 , where $\frac{W}{l_{s}}=4.0$.

The only design variables not examined below are the stacking sequences of the skin and blade laminates. Recall that these variables impact both the infiltration time, through the permeability of the preform, and the buckling ratios of the structural elements. The effects of the stacking sequences will be observed later in the optimization results.

\subsubsection{Manufacturing Analysis}

The first set of results will show how each of the design variables impacts the manufacturing analysis of the blade-stiffened panel, specifically, the resin infiltration time and the filled percentage of the preform. Instances were the resin gels prior to complete infiltration of the preform will also be noted.

\subsubsection{Effect of Manufacturing Variables}

As discussed in Chapter 2, temperature plays a large role in the RFI process. Figure 4.1 illustrates the results of fill time and percent of the cross-section filled as a function of varying the cure temperature from $80^{\circ} \mathrm{C}$ to $200^{\circ} \mathrm{C}$ while fixing all other design 
variables. The "•" symbol in the plot indicates the point at which the resin gels. As the temperature increases, the infiltration time rapidly falls, leveling off at approximately $110^{\circ} \mathrm{C}$, and then begins to rise slightly before the resin gels at $115^{\circ} \mathrm{C}$. Above this temperature, the percentage of the preform infiltrated with resin decreases quickly. Therefore, for the baseline set of design variables, the cure temperature must remain below $115^{\circ} \mathrm{C}$ for complete infiltration of the part.

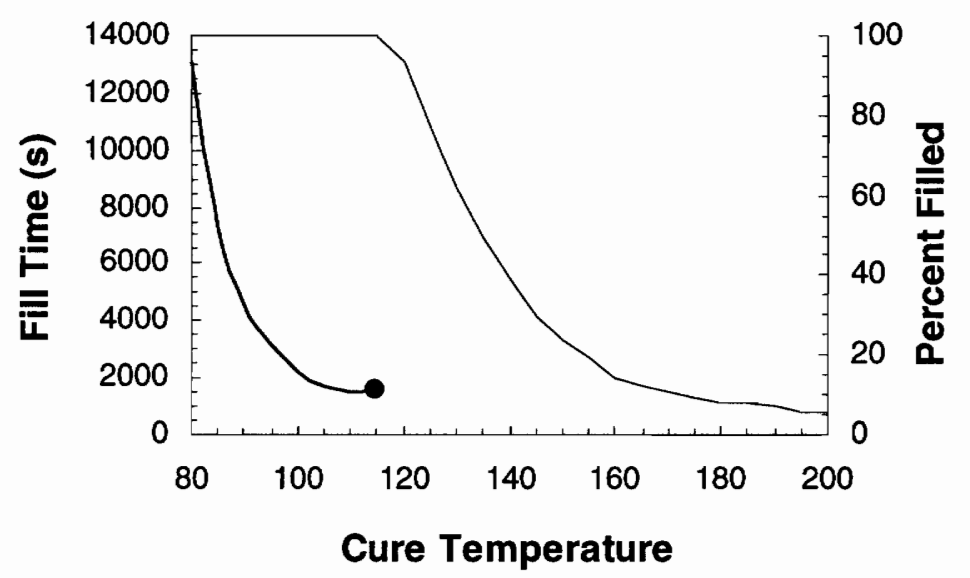

Figure 4.1 Effect of Temperature on Manufacturing

The effect of compaction pressure, the other manufacturing variable, on the fill time and volume filled is shown in Figure 4.2. It is clear from the figure that the resin will gel before infiltration is complete if the pressure falls below $800 \mathrm{kPa}$. Above this value, increasing the pressure reduces the infiltration time significantly, but the effect begins to taper off around $1,500 \mathrm{kPa}$. 


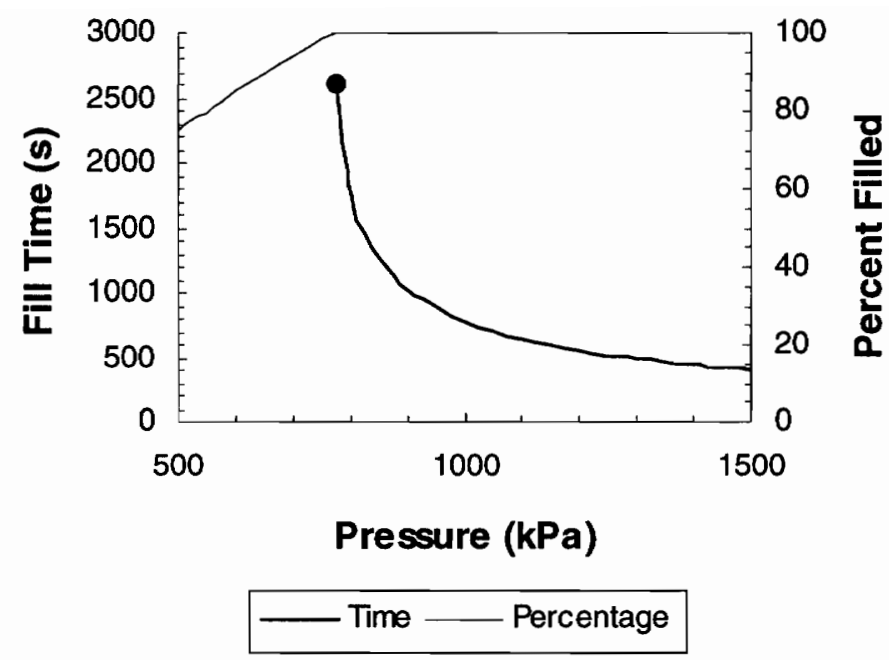

Figure 4.2 Effect of Pressure on Manufacturing

Different pressure/temperature combinations also greatly affect the manufacturing performance of the panel. Figure 4.3 shows the infiltration times for varying pressure at several temperatures. The figure shows that for cure temperatures of $100^{\circ}$ and $110^{\circ} \mathrm{C}$, complete infiltration is attained for all pressures in the range plotted. The plot also indicates that, for the baseline panel geometry, a temperature of $110^{\circ} \mathrm{C}$ gives the lowest infiltration times for pressures under roughly $900 \mathrm{kPa}$. For higher pressures, however, the nominal temperature of $120^{\circ} \mathrm{C}$ gives the shortest times. As the temperature increases above this value, the infiltration times begin to increase, and the available pressure range over which the resin will not completely fill the preform before it gels becomes smaller, with the minimum allowable pressure increasing. For example, when the temperature is $130^{\circ} \mathrm{C}$, the pressure must be nearly $1,500 \mathrm{kPa}$ for complete infiltration to occur. 


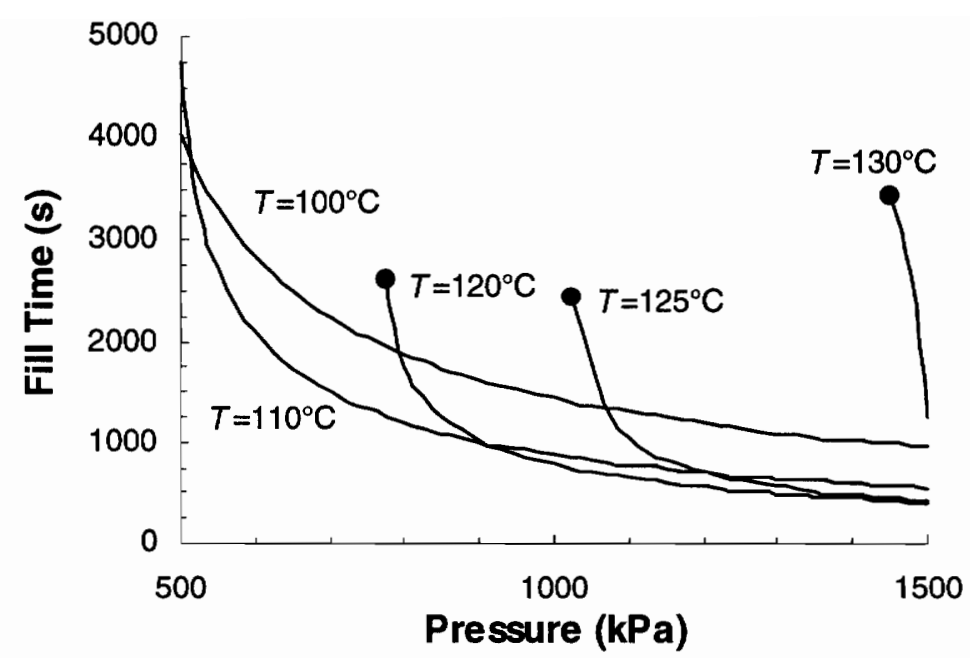

Figure 4.3 Effect of Pressure on Manufacturing at Several Temperatures

\subsubsection{Effect of Geometric Variables}

From inspection of the panel cross-section alone, it is clear that changing the blade height will greatly impact the resin infiltration time. The results in Figure 4.4, which shows the fill time and percent filled as a function of the blade height, affirm this observation.

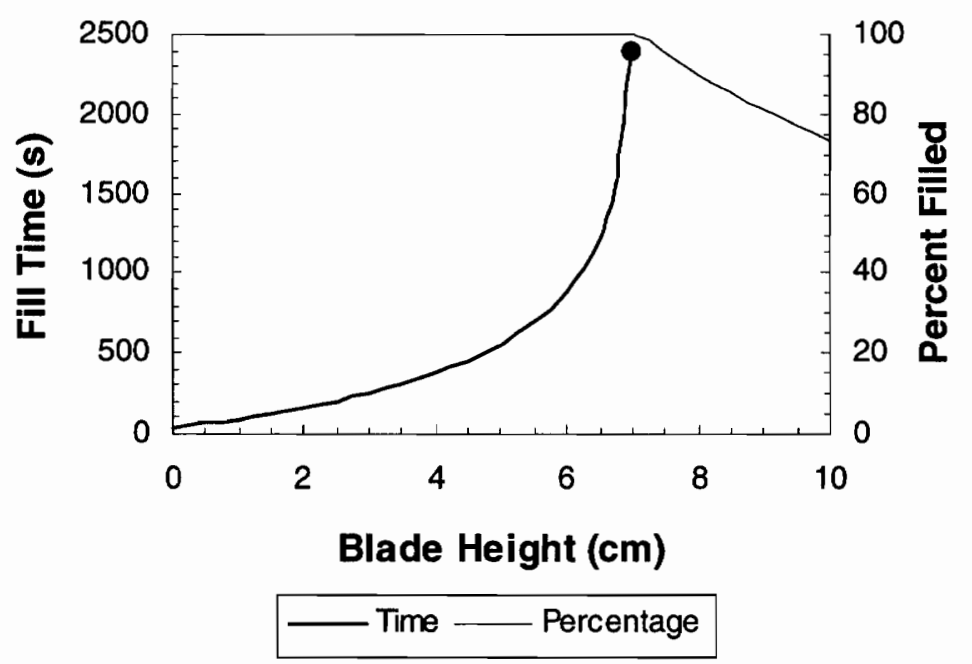

Figure 4.4 Effect of Blade Height on Manufacturing 
From the plot, it can be seen that the fill time increases as the blade height increases, as expected. However, for the temperature and pressure values fixed at their nominal values as given in Table 4.2, around $7 \mathrm{~cm}$ the blade becomes too tall for the resin to infiltrate before the resin has gelled.

Figure 4.5 plots the effect of the skin width on fill time. The figure shows that, for complete infiltration to occur, the skin width must be greater than approximately $26 \mathrm{~cm}$ for the baseline design. Above this value, the fill time sharply drops before beginning to level off. The "o" symbol is used in the plot to recall that the skin width cannot fall below the flange width of $6.10 \mathrm{~cm}$, which is a geometric constraint on the problem.

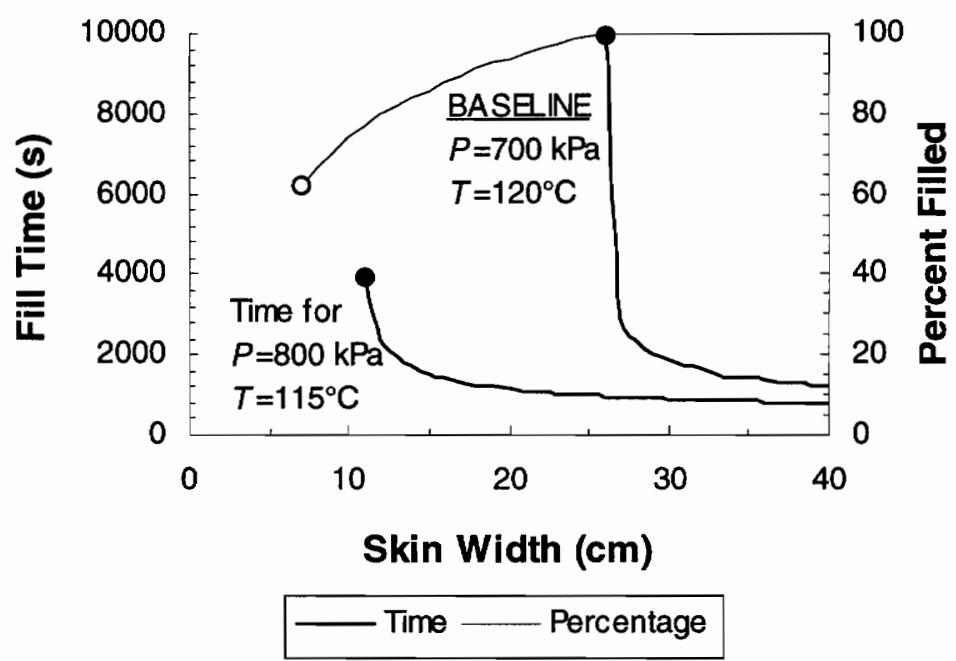

Figure 4.5 Effect of Skin Width on Manufacturing

Again, this plot is for fixed values of the manufacturing variables. For other combinations of temperature and pressure, it is possible to fully infiltrate panels with skins smaller than $26 \mathrm{~cm}$ in width. For example, the fill time for a pressure and temperature of $800 \mathrm{kPa}$ and $115^{\circ} \mathrm{C}$, respectively, is also shown in Figure 4.5. This time, the minimum skin width for complete infiltration is about $11 \mathrm{~cm}$. 
As in Chapter 3, the flange width is expressed as a percentage of the skin width to ensure that it does not exceed that value. Figure 4.6 illustrates the effect of changing the flange-to-skin width ratio.

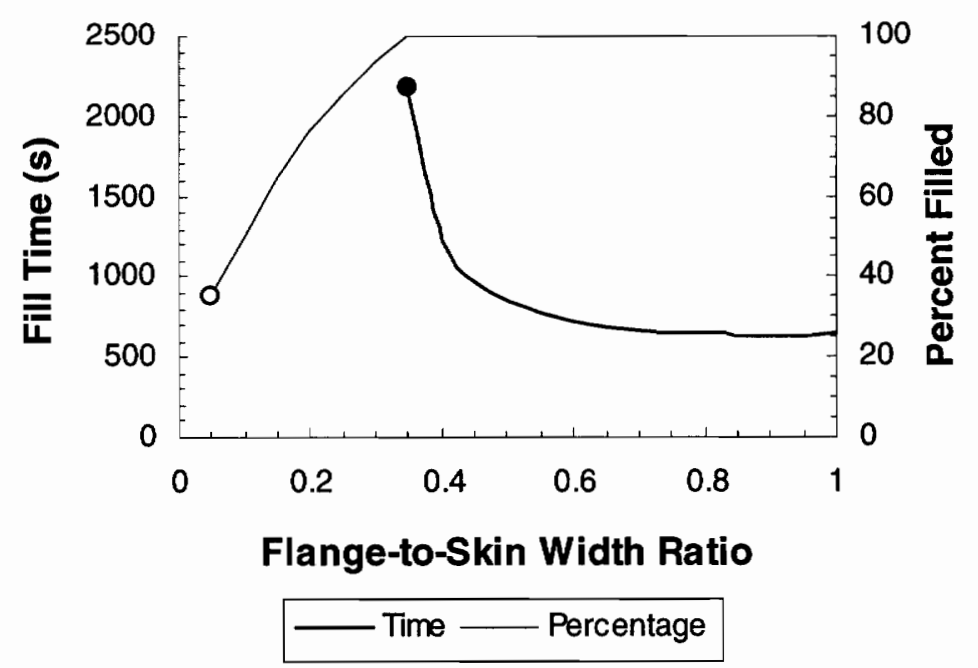

Figure 4.6 Effect of Flange-to-Skin Width Ratio on Manufacturing

The plot indicates the lowest allowable value the ratio can attain, keeping all other design variables fixed, for complete infiltration of the preform. This value is around 0.35 . The "o" symbol in Figure 4.6 is used to represent the geometric constraint that the flange width cannot fall below the blade thickness of $0.957 \mathrm{~cm}$.

\subsubsection{Structural Analysis}

The set of design variables chosen for the problem also affect the structural performance and the geometry of the structure. Effects of geometric variables on structural performance have been researched reasonably extensively in the literature. However, effects of manufacturing-related variables on structures are less obvious. The effects of parametrically varying the design variables on the structural response of the 
panel will be discussed below. These effects will be observed in the form of changes in the panel mass and the skin and stiffener buckling ratios.

\subsubsection{Panel Mass}

As mentioned in Chapter 2, temperature has no direct impact on structural performance. Pressure, on the other hand, changes the ply thickness by altering the fiber volume fraction, thereby influencing the panel mass. The effect of pressure on the panel mass is shown in Figure 4.7 for both the unit cell and a panel with multiple stiffeners configurations mentioned earlier (See Section 4.2.2.1 for the motivation for changing the panel mass computation.). The plots reveal that, although increasing pressure decreases the mass for both configurations, the change is not substantial.
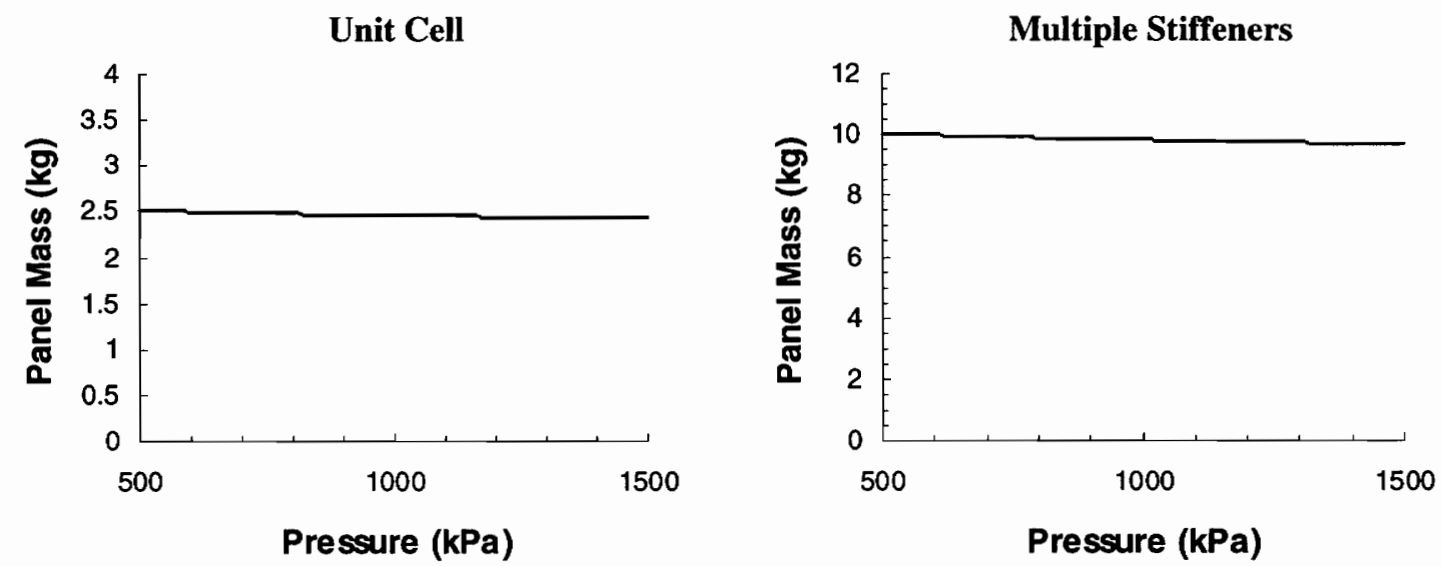

Figure 4.7 Effect of Pressure on Panel Mass

The geometric variables, on the other hand, have an obvious effect on the panel mass. Increasing the size of these elements increases the mass linearly. Figures 4.8 and 4.9 demonstrate these effects for the blade height and flange width, respectively. The "o" symbols in Figure 4.9 denote the minimum allowable width ratio value as was indicated in Figure 4.6. 
Unit Cell

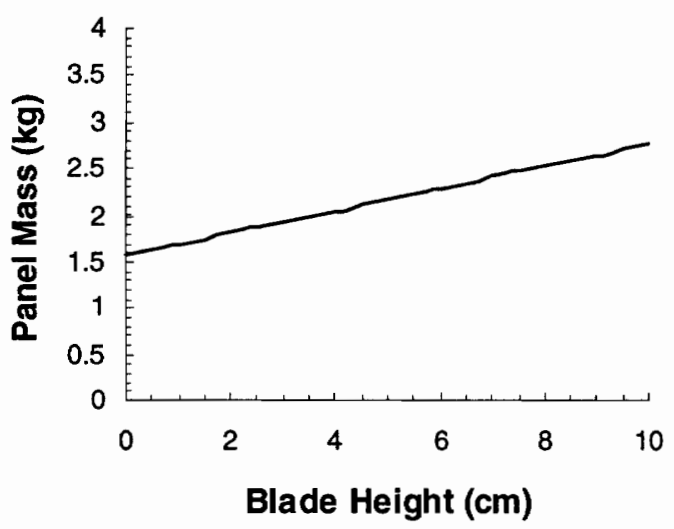

Multiple Stiffeners

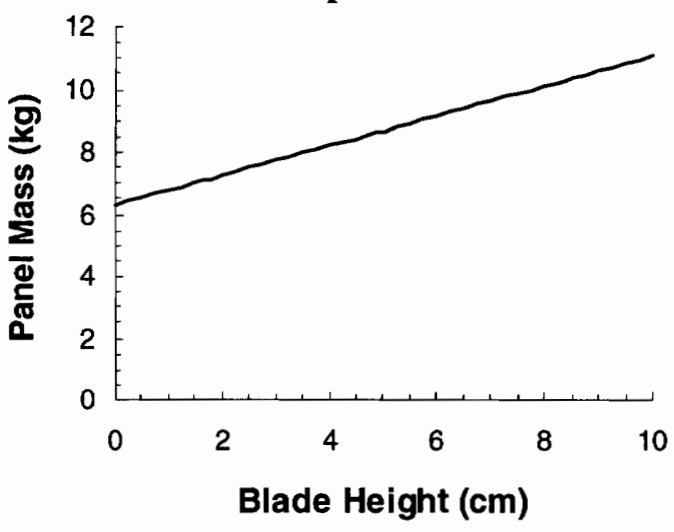

Figure 4.8 Effect of Blade Height on Panel Mass

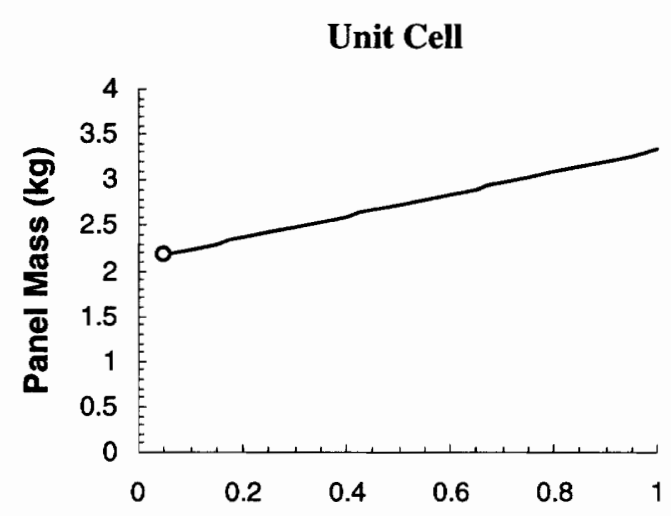

Flange-to-Skin Width Ratio

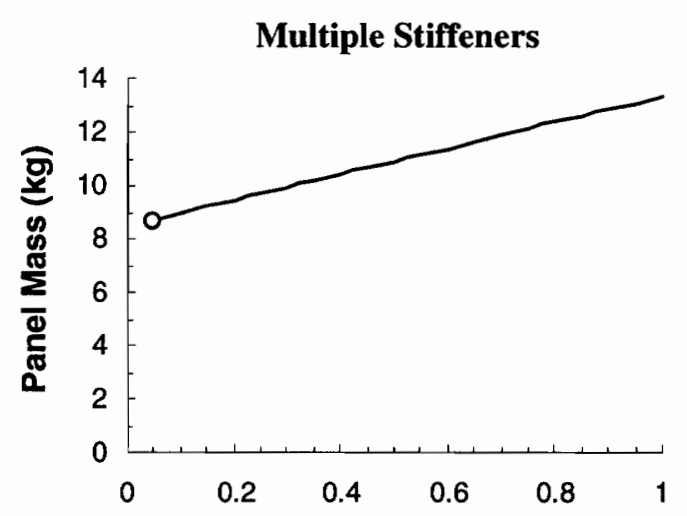

Flange-to-Skin Width Ratio

Figure 4.9 Effect of Flange-to-Skin Width Ratio on Panel Mass

The consequence of the redefinition of the panel mass is that the above trends of increasing mass with increasing geometric variable hold true for the skin width only in the unit cell configuration. As illustrated in Figure 4.10, widening the skin raises the mass of the unit cell linearly, as expected. However, when the panel consists of multiple stiffeners in a fixed width, increasing $l_{s}$ decreases the total number of stiffeners. This, in turn, reduces the mass. As in Figure 4.5, the "o" symbols in the plots are used to indicate the minimum allowable skin width of $6.10 \mathrm{~cm}$. 

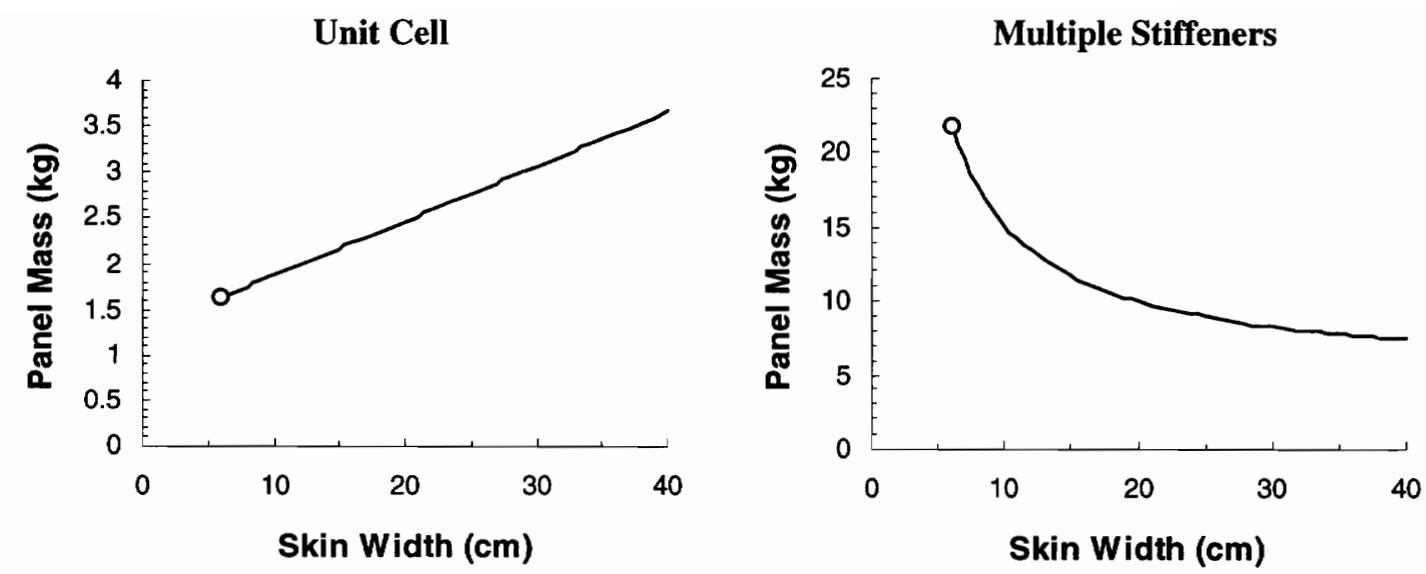

Figure 4.10 Effect of Skin Width on Panel Mass

\subsubsection{Skin and Stiffener Buckling}

The buckling ratios of the skin and stiffener elements depend on both the actual load each element carries and critical value of this load. Changes in the cross-sectional geometry and the material properties of the panel affect both the loading distribution through Eq. 2.40 and the critical buckling loads through Eqs. 2.51 and 2.52. Recall that for the elements not to buckle, the ratio of the actual load to its critical value must be less than one.

As previously discussed, pressure governs the thicknesses of the skin and blade laminates as well as the material properties of the structural elements. Figure 4.11 illustrates the effect of varying pressure on the buckling behavior of the panel. From the figure, it can be seen that the stiffener buckling ratio remains fairly constant across the pressure range with the actual loading remaining just under one-half of its critical value. The skin buckling constraint, on the other hand, is always violated with the actual loading approaching just over twice its critical value at a pressure of $1,500 \mathrm{kPa}$. Therefore, there 
is no pressure that is sufficient to satisfy the buckling criteria while maintaining all other design variables at their baseline values.

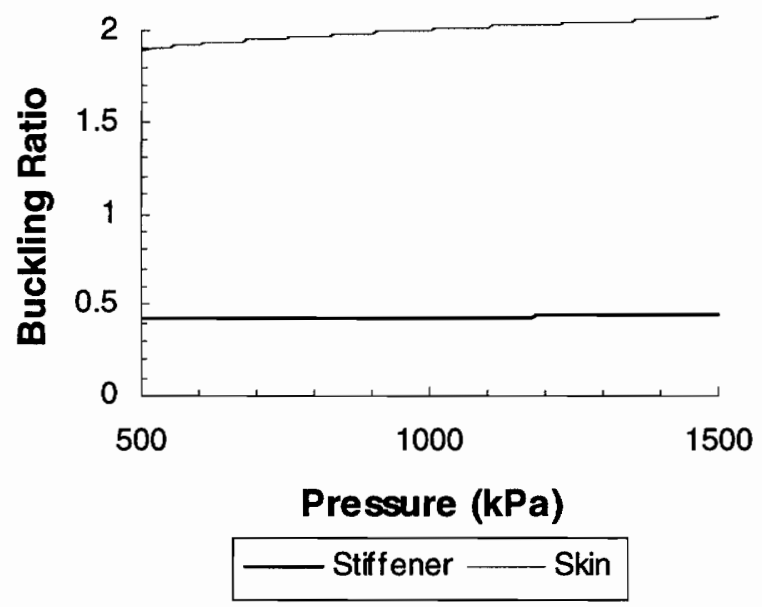

Figure 4.11 Effect of Pressure on Panel Buckling

The effect of different blade heights is presented in Figure 4.12. The plot shows that the blade height greatly affects the stiffener buckling characteristics. Increasing $h_{b}$ rapidly decreases the buckling ratio (Eq. 3.6), but its value needs to be above approximately $5.5 \mathrm{~cm}$ for the stiffener not to buckle. However, in reality, tall blades are more susceptible to buckling. Recall from Section 2.3.3.2 that the stiffener is treated as a column undergoing axial compression. Thus, the higher the value of $h_{b}$, the higher the stiffener moment of inertia (Eq. 2.53). The increased moment of inertia raises the critical buckling load for the stiffener (Eq. 2.52), which, in turn, reduces the buckling ratio as shown in the figure. For an improved structural model, the blade laminate could be further modeled as a plate simply-supported on three sides, and a third critical buckling load could be computed. Regardless, as will be shown later, the optimal blade heights are significantly shorter than that of the baseline case, and this refined model is not considered. 
The skin buckling ratio, on the other hand, is not as greatly affected by $h_{b}$. Nevertheless, the skin consistently buckles over the range plotted because the blade is not tall enough to reduce the share of the loading taken by the skin.

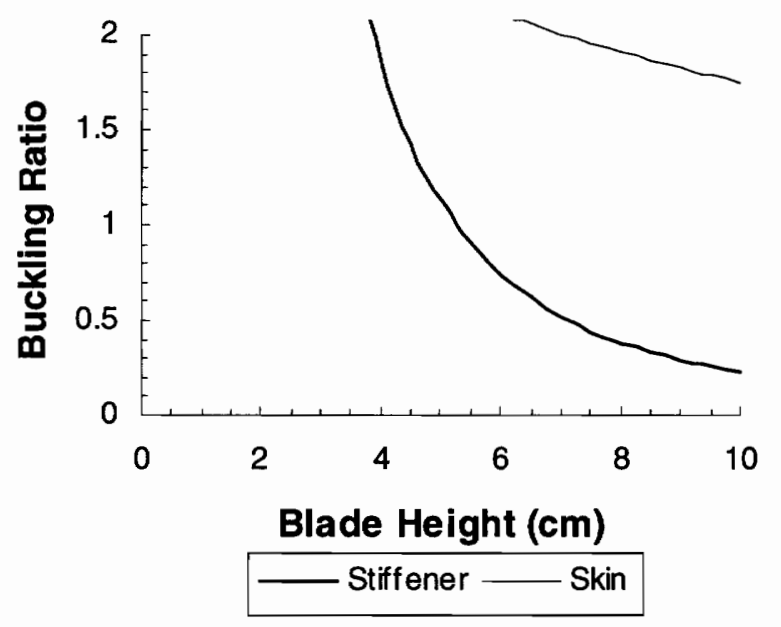

Figure 4.12 Effect of Blade Height on Panel Buckling

If, however, the width of the skin is changed while keeping the blade height constant, Figure 4.13 shows that both buckling constraints can be satisfied. The stiffener does not buckle over the range, and increasing the skin width improves its constraint margin. The skin can also be made not to buckle as long as $l_{s}$ remains below about $16 \mathrm{~cm}$. The " $\mathrm{O}$ " symbol appears in the plot once again as a reminder of the geometrical limitations of the skin width.

The consequences of changing the width of the flange are illustrated in Figure 4.14. Once again, the value of $r_{\text {stiff }}$ remains below one over the plotted range of the flange-to-skin width ratio. Notice also that it achieves a minimum when $\frac{l_{f}}{l_{s}}$ is approximately 0.4 . The skin, however, will buckle at any width ratio below roughly 0.5 . 


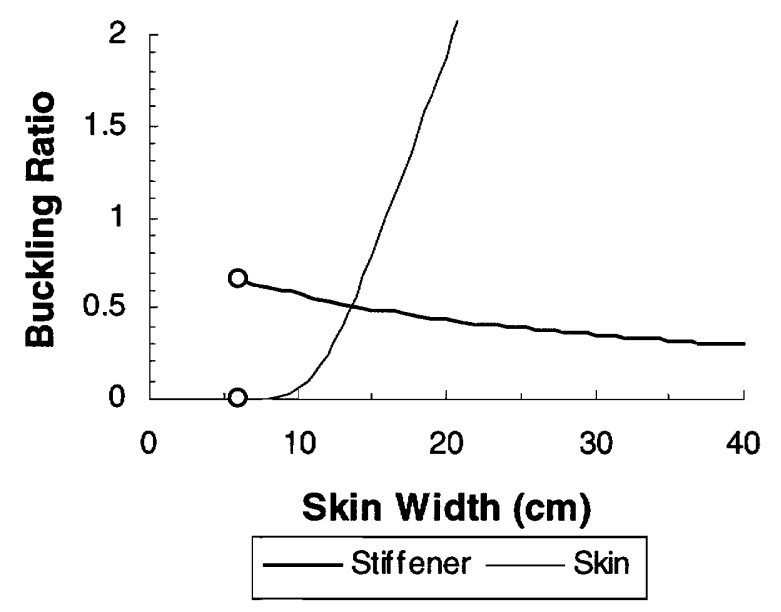

Figure 4.13 Effect of Skin Width on Panel Buckling

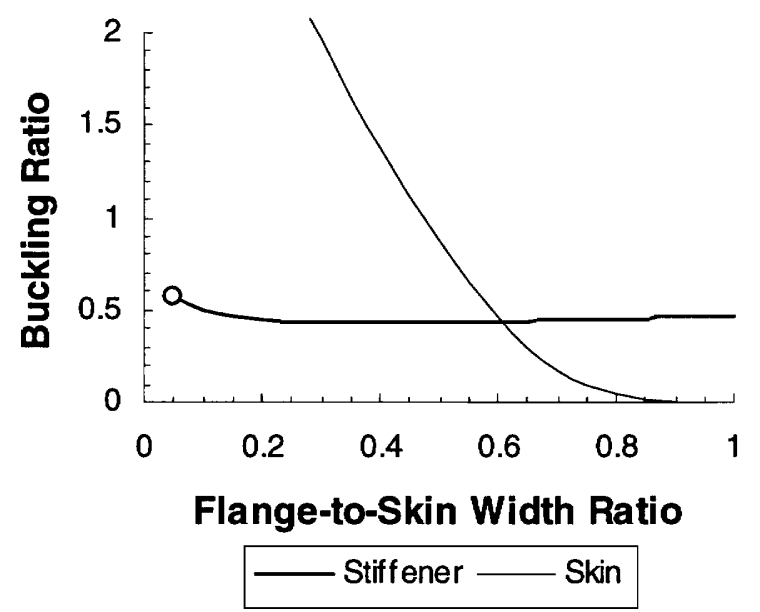

Figure 4.14 Effect of Flange-to-Skin Width Ratio on Panel Buckling

Finally, it is also desirable to observe the effects of varying the applied loading itself. Once more, the stiffener buckling constraint is not violated for the loading range plotted in Figure 4.15. The figure also reveals that in order for the skin not to buckle, the applied end-shortening load to the panel must remain below approximately $400 \mathrm{kN}$. 


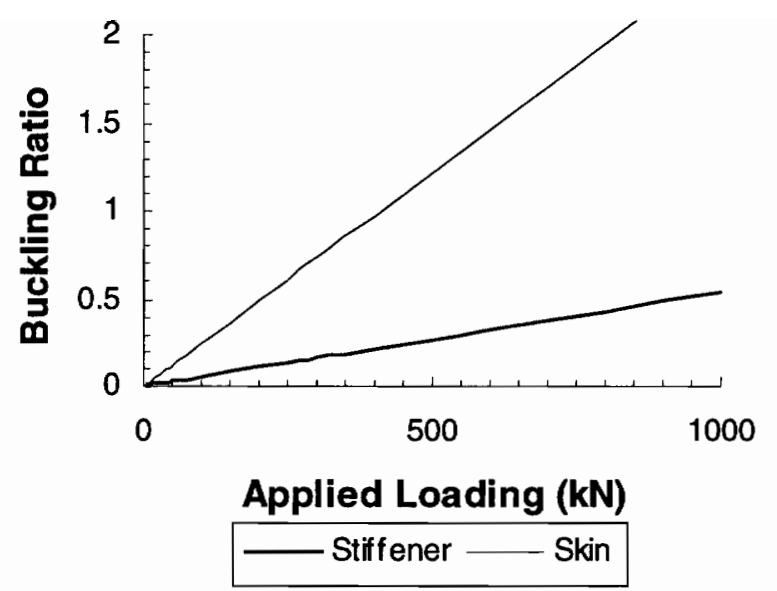

Figure 4.15 Effect of Loading on Panel Buckling

All of the results in this subsection have shown that the buckling constraint of the skin element is the difficult/critical one to satisfy. The stiffener buckling constraint is always satisfied within the ranges of the design variables investigated here.

\subsection{Optimization Results}

The subsections that follow present the optimization results obtained with the genetic algorithm. Optimal designs for the structures and manufacturing problems alone will be shown first. That will be followed by optima for the multiobjective problem. Table 4.3 gives the GA parameters used for the optimization. These are the population size ( $\Pi$ ), the mutation probability $\left(p_{m u t}\right)$, and the permutation probability $\left(p_{p e r}\right)$.

Table 4.3 Genetic Algorithm Parameters

\begin{tabular}{ccc}
\hline Population Size & Mutation Probability & $\begin{array}{c}\text { Permutation Probability } \\
\Pi \Pi\end{array}$ \\
$\boldsymbol{p}_{\text {mut }}$ & $\boldsymbol{p}_{\text {per }}$ \\
\hline 50 & 0.001 & 0.50 \\
\hline
\end{tabular}

Table 4.4 gives the weighting and penalty/bonus parameters used to form the objective function $\Psi$ from Eq. 3.17. Recall that $\beta_{1}$ and $\beta_{2}$ are the weighting parameters 
for the panel mass and infiltration times, respectively, as was shown in Eq. 3.5. The mass is penalized by $\chi_{1}$ for violations in the buckling constraints and given a bonus of $\varepsilon_{1}$ for their satisfaction. The infiltration times of panel designs that are not fully filled are penalized by $\chi_{2}$. The augmented mass and infiltration times were given in Eqs. 3.15 and 3.16 , respectively.

Table 4.4 Objective Function Weighting and Constraint Penalty/Bonus Parameters

\begin{tabular}{ccccc}
\hline $\begin{array}{c}\text { Structural } \\
\text { Objective } \\
\text { Weighting }\end{array}$ & $\begin{array}{c}\text { Manuf. } \\
\text { Objective } \\
\text { Weighting }\end{array}$ & $\begin{array}{c}\text { Structural } \\
\text { Constraint } \\
\text { Violation } \\
\text { Penalty }\end{array}$ & $\begin{array}{c}\text { Manuf. } \\
\text { Constraint } \\
\text { Violation } \\
\text { Penalty }\end{array}$ & $\begin{array}{c}\text { Structural } \\
\text { Constraint } \\
\text { Satisfaction } \\
\text { Bonus }\end{array}$ \\
$\beta_{1}$ & $\beta_{2}$ & $\chi_{1}$ & $\chi_{2}$ & $\varepsilon_{1}$ \\
\hline 0.125 & 0.002 & 4 & 10 & 0.005 \\
\hline
\end{tabular}

\subsubsection{Role of Pressure as a Design Variable}

Although the compaction pressure has a profound effect on the manufacturing analysis of the panel as shown in Figure 4.2, the results in Figures 4.7 and 4.11 indicate that it has only a slight effect on the structural performance. In addition, preliminary optimization results reveal that the optimizer will increase the value of $P$ to whatever maximum allowable value it is given. In the actual manufacturing process, however, there are practical limits to the amount of pressure used. Taking all of this into account, pressure will be removed as a design variable in the genetic algorithm. Nevertheless, optimization results will be presented for different values of $P$ in order to observe the effects. 


\subsubsection{Structural Optima}

The structural optimization problem entails minimization of the panel mass while satisfying the skin and stiffener buckling constraints. Results will also be presented for different compaction pressures and loads to study the effects of varying these quantities parametrically. Recall that the baseline values of the pressure and load are $700 \mathrm{kPa}$ and $800 \mathrm{kN}$, respectively.

\subsubsection{Problem Reformulation}

As mentioned in Section 4.1, preliminary results necessitated the reformulation of the structural optimization problem. Using the mass of the unit cell alone as the objective to be minimized, the optimizer would "collapse" the widths of the skin and flange to a value equal to the blade thickness. Thus, the unit cell consisted solely of the blade, which would carry the entire load.

In order to prevent this from occurring, the panel mass was redefined incorporating multiple stiffeners in a fixed panel width as given by Eq. 4.1. The encoding of $l_{s}$ for the GA is also changed to reflect the new formulation. Eq. 3.10 now becomes

$$
l_{s}=t_{b}+\frac{\Omega_{5}}{242}\left(W-t_{b}\right),
$$

where $\Omega_{5}$ is given by Eq. 3.8, and 242 is the maximum value $\Omega_{5}$ can take. This allows the skin width vary between $t_{b}$ and $W$.

Nonetheless, when the optimizer was given the problem of minimizing the new mass, it would expand the width of the skin to a chosen value of $W$. The flange would 
also be nearly as wide since the width of the skin element used in the buckling analysis is the difference between $l_{s}$ and $l_{f}$. The resulting minimum-mass panel would therefore contain only one stiffener. The blade height, however, is comparable to the results that will be shown in the subsequent sections.

From this outcome, it is clear that redefining the mass is not enough. Constraints must also be placed on the geometric design variables to avert the above scenario. These constraints are

$$
\begin{aligned}
& g_{4}=\frac{l_{f}}{t_{b}}-7 \leq 0 \\
& g_{5}=2-\frac{l_{f}}{t_{b}} \leq 0 \\
& g_{6}=\frac{l_{f}}{l_{s}}-\frac{2}{3} \leq 0
\end{aligned}
$$

The first two restrict the value of the flange width to a range of $2 t_{b} \leq l_{f} \leq 7 t_{b}$, and the third ensures that space equal to at least $0.5 l_{f}$ remains between individual stiffeners.

Recall that the GA is an unconstrained optimization procedure. Thus, the constraint violations are handled by a penalty approach. From Eq. 3.15, the quantity $M^{*}$ gives the mass function penalized by violations of the buckling constraints. $M^{*}$ is further penalized if the constraints in Eq. 4.2 are not satisfied. This new augmented panel mass is given by

$$
M^{* *}=M^{*}+\chi_{3} \max \left(g_{4}, g_{5}, g_{6}, 0\right),
$$

where $\chi_{3}$ is the penalty parameter with a value of 0.6 . 


\subsubsection{Effect of Pressure Variation}

Optimization results for minimum-mass panel designs at four different pressures are shown in Tables 4.5-4.8. The structural optimum for the nominal values of pressure (700 kPa) and loading $(800 \mathrm{kN})$ is given in Table 4.6. Comparing these results with the baseline design in Table 4.2, the skin for the structural optimum is $37 \%$ wider, the flange is $0.49 \%$ wider, and the blade is $34 \%$ shorter.

The optimum stacking sequences for the skin and blade laminates are $\left[ \pm 45^{\circ}{ }_{10}\right]_{s}$ and $\left[0^{\circ}{ }_{80}\right]$, respectively. The all-zero-ply blade gives the stiffener the maximum stiffness along the loading direction so that it can carry the majority of the load. Meanwhile, the $\pm 45^{\circ}$ plies keep the skin just under the verge of buckling. This supports the results from Section 4.1.2.2 that the skin buckling constraint is the critical one to satisfy.

Comparing the four pressure cases, the skin and blade thicknesses decrease with increasing pressure, as expected. The skin and flange widths also decrease with pressure, although this effect begins to taper off when going from 900 to $1,100 \mathrm{kPa}$. On the other hand, adding more pressure raises the blade height to account for the smaller skin and flange. The net result of the above trends in the geometric variables produces an overall decrease in the panel mass with increasing pressure. Nevertheless, a savings of only $1.3 \%$ is achieved when going from $700 \mathrm{kPa}$, the baseline value, to $1,100 \mathrm{kPa}$. In addition, the skin and blade stacking sequences are invariable with pressure, and all constraints are satisfied, with the skin buckling ratio being critical or near-critical in all cases. Note that the stiffener buckling ratio is also nearly critical for 500 and $900 \mathrm{kPa}$. 
Table 4.5 Structural Optimum

$(P=500 \mathrm{kPa} ; F=800 \mathrm{kN})$

\section{Geometric Variables}

$\begin{array}{ll}l_{s} & 29.15 \mathrm{~cm} \\ l_{f} & 6.78 \mathrm{~cm} \\ h_{b} & 4.90 \mathrm{~cm} \\ t_{s} & 0.485 \mathrm{~cm} \\ t_{b} & 0.971 \mathrm{~cm}\end{array}$

Material Variables

$\begin{array}{ll}\Theta_{s} & 22222 \\ \Theta_{b} & 1111111111\end{array}$

Manufacturing Variables

$P \quad 500 \mathrm{kPa}$

Structural Analysis

\begin{tabular}{ll}
$F$ & $800 \mathrm{kN}$ \\
$M$ & $7.68 \mathrm{~kg}$ \\
$r_{\text {stiff }}$ & 0.997 \\
$r_{\text {skin }}$ & 0.994 \\
$l_{f} / t_{b}$ & 6.99 \\
$l_{f} / l_{s}$ & 0.233 \\
\hline
\end{tabular}

Table 4.7 Structural Optimum $(P=900 \mathrm{kPa} ; F=800 \mathrm{kN})$

Geometric Variables

$\begin{array}{ll}l_{s} & 27.48 \mathrm{~cm} \\ l_{f} & 6.06 \mathrm{~cm} \\ h_{b} & 5.00 \mathrm{~cm} \\ t_{s} & 0.474 \mathrm{~cm} \\ t_{b} & 0.947 \mathrm{~cm}\end{array}$

Material Variables

$\begin{array}{ll}\Theta_{s} & 22222 \\ \Theta_{b} & 1111111111\end{array}$

Manufacturing Variables

$P \quad 900 \mathrm{kPa}$

Structural Analysis

$\begin{array}{ll}F & 800 \mathrm{kN} \\ M & 7.61 \mathrm{~kg} \\ r_{\text {stiff }} & 0.993 \\ r_{\text {skin }} & 0.994 \\ l_{f} / t_{b} & 6.39 \\ l_{f} / l_{s} & 0.221\end{array}$

Table 4.6 Structural Optimum $(P=700 \mathrm{kPa} ; F=800 \mathrm{kN})$

Geometric Variables

$\begin{array}{ll}l_{s} & 27.82 \mathrm{~cm} \\ l_{f} & 6.13 \mathrm{~cm} \\ h_{b} & 5.00 \mathrm{~cm} \\ t_{s} & 0.479 \mathrm{~cm} \\ t_{b} & 0.957 \mathrm{~cm}\end{array}$

Material Variables

$\begin{array}{ll}\Theta_{s} & 22222 \\ \Theta_{b} & 1111111111\end{array}$

Manufacturing Variables

$P \quad 700 \mathrm{kPa}$

Structural Analysis

$\begin{array}{ll}F & 800 \mathrm{kN} \\ M & 7.65 \mathrm{~kg} \\ r_{\text {stiff }} & 0.983 \\ r_{\text {skin }} & 0.996 \\ l_{f} / t_{b} & 6.40 \\ l_{f} / l_{s} & 0.220\end{array}$

Table 4.8 Structural Optimum $(P=1,100 \mathrm{kPa} ; F=800 \mathrm{kN})$

Geometric Variables

$\begin{array}{ll}l_{s} & 27.47 \mathrm{~cm} \\ l_{f} & 6.05 \mathrm{~cm} \\ h_{b} & 5.05 \mathrm{~cm} \\ t_{s} & 0.470 \mathrm{~cm} \\ t_{b} & 0.939 \mathrm{~cm}\end{array}$

Material Variables

$\begin{array}{ll}\Theta_{s} & 22222 \\ \Theta_{b} & 1111111111\end{array}$

Manufacturing Variables

$P \quad 1,100 \mathrm{kPa}$

Structural Analysis

\begin{tabular}{ll}
$F$ & $800 \mathrm{kN}$ \\
$M$ & $7.58 \mathrm{~kg}$ \\
$r_{\text {stiff }}$ & 0.973 \\
$r_{\text {skin }}$ & 1.00 \\
$l_{f} / t_{b}$ & 6.45 \\
$l_{f} / l_{s}$ & 0.220 \\
\hline
\end{tabular}




\subsubsection{Effect of Load Level}

Tables 4.9 and 4.10 present optimization results for two additional loadings. The compaction pressure is maintained at $700 \mathrm{kPa}$. Recall that the data for $800 \mathrm{kN}$ was already given in Table 4.6. The values of $t_{s}$ and $t_{b}$ do not change here, as previously discussed, since the pressure remains the same in each case. Increasing the value of $F$ causes $l_{s}$ to decrease as it did with increasing pressure. However, the blade grows taller to accept the added loading. In addition, the flange increases in width to decrease the quantity $l_{s}-l_{f}$, thereby reducing the portion of the load the skin element has to support. These increases in $h_{b}$ and $l_{f}$, as well as the reduction in $l_{s}$, which increases the total number of stiffeners, cause the panel mass to increase with $F$. Thus, if the loading were decreased to $600 \mathrm{kN}$ from the baseline value of $800 \mathrm{kN}$, there would be a mass savings of $6.0 \%$, but increasing the load to $1,000 \mathrm{kN}$ pays a mass penalty of $5.5 \%$.

As before, all the constraints are satisfied for each loading case. The skin buckling constraint is, again, the critical one. The stacking sequences remain unchanged from those given in the pressure cases. This shows that for the structural optimization problem, $\boldsymbol{\Theta}_{s}$ and $\Theta_{b}$ are insensitive to both pressure and loading.

\subsubsection{Manufacturing Analysis of Structural Optima}

The structural optima presented in Tables 4.5-4.10 are also analyzed with the onedimensional RFI model. To do this, however, a cure temperature must be selected since it is not a design variable in the structural optimization. For convenience, the nominal value of $120^{\circ} \mathrm{C}$ from Table 4.2 is chosen. The infiltration times for each of the structural optima 
for each pressure and loading case are summarized in Table 4.11. Note that each entry in this table also gives the table where the values of the geometric and material design variables, as well as the results of the structural analyses, can be found. Recall that the values of these design variables differ from case to case. All designs in the table are completely infiltrated. The "--" entries denote cases for which optimizations were not performed.

Table 4.9 Structural Optimum $(P=700 \mathrm{kPa} ; F=600 \mathrm{kN})$

\begin{tabular}{cc}
\hline Geometric Variables & \\
\hline$l_{s}$ & $30.47 \mathrm{~cm}$ \\
$l_{f}$ & $5.96 \mathrm{~cm}$ \\
$h_{b}$ & $4.40 \mathrm{~cm}$ \\
$t_{s}$ & $0.479 \mathrm{~cm}$ \\
$t_{b}$ & $0.957 \mathrm{~cm}$
\end{tabular}

Material Variables

$\begin{array}{ll}\Theta_{s} & 22222 \\ \Theta_{b} & 1111111111\end{array}$

Manufacturing Variables

$P \quad 700 \mathrm{kPa}$

Structural Analysis

\begin{tabular}{ll}
$F$ & $600 \mathrm{kN}$ \\
$M$ & $7.19 \mathrm{~kg}$ \\
$r_{\text {stiff }}$ & 0.978 \\
$r_{\text {skin }}$ & 0.999 \\
$l_{f} / t_{b}$ & 6.23 \\
$l_{f} / l_{s}$ & 0.196 \\
\hline
\end{tabular}

Table 4.10 Structural Optimum $(P=700 \mathrm{kPa} ; F=1,000 \mathrm{kN})$

\begin{tabular}{cc}
\hline Geometric Variables & \\
\hline$l_{s}$ & $26.16 \mathrm{~cm}$ \\
$l_{f}$ & $6.41 \mathrm{~cm}$ \\
$h_{b}$ & $5.50 \mathrm{~cm}$ \\
$t_{s}$ & $0.479 \mathrm{~cm}$ \\
$t_{b}$ & $0.957 \mathrm{~cm}$
\end{tabular}

Material Variables

$\begin{array}{ll}\Theta_{s} & 22222 \\ \Theta_{b} & 1111111111\end{array}$

Manufacturing Variables

$P \quad 700 \mathrm{kPa}$

Structural Analysis

\begin{tabular}{ll}
$F$ & $1,000 \mathrm{kN}$ \\
$M$ & $8.07 \mathrm{~kg}$ \\
$r_{\text {stiff }}$ & 0.985 \\
$r_{\text {skin }}$ & 0.998 \\
$l_{f} / t_{b}$ & 6.70 \\
$l_{f} / l_{s}$ & 0.245 \\
\hline
\end{tabular}


Table 4.11 Infiltration Times of Structural Optima at $T=120^{\circ} \mathrm{C}$

\begin{tabular}{|c|c|c|c|}
\hline$P(F \rightarrow$ & $600 \mathrm{kN}$ & 800 kN & $1,000 \mathrm{kN}$ \\
\hline $500 \mathrm{kPa}$ & -- & $\begin{array}{c}\text { Table } 4.5 \\
1,034 \mathrm{~s}\end{array}$ & -- \\
\hline $700 \mathrm{kPa}$ & $\begin{array}{c}\text { Table } 4.9 \\
494 \mathrm{~s}\end{array}$ & $\begin{array}{c}\text { Table } 4.6 \\
693 \mathrm{~s}\end{array}$ & $\begin{array}{c}\text { Table } 4.10 \\
940 \mathrm{~s}\end{array}$ \\
\hline $900 \mathrm{kPa}$ & -- & $\begin{array}{c}\text { Table } 4.7 \\
463 \mathrm{~s}\end{array}$ & -- \\
\hline $1,100 \mathrm{kPa}$ & -- & $\begin{array}{c}\text { Table } 4.8 \\
362 \mathrm{~s}\end{array}$ & -- \\
\hline
\end{tabular}

From the table, it can be seen that for the $800 \mathrm{kN}$ loading cases, the infiltration times decrease with increasing pressure, as was true with the panel masses for these cases, despite the fact that designs obtained for higher pressures have slightly thinner and taller stiffeners. A time savings of $65 \%$ is achieved in going from 500 to $1,110 \mathrm{kPa}$. For the fixed pressure $(700 \mathrm{kPa})$ with variable loading cases, on the other hand, raising the load also raises the time for infiltration, with a penalty of $90 \%$ when the load increases from 600 to $1,000 \mathrm{kN}$.

\subsubsection{Manufacturing Optima}

The optimum designs for the manufacturing optimization are given in Tables 4.124.15 for four different compaction pressures. Note that the loading is not varied as it has no effect on the infiltration process. From these results it can be seen that the width of the skin is extended to equal the entire width of the panel, and the blade is reduced to essentially nothing ( $0.05 \mathrm{~cm}$ is the lowest value $h_{b}$ is allowed to take in the GA). The flange width reduces to a value of $2.82 \mathrm{~cm}$ to provide an optimal flange-to-skin width ratio of 0.035 ), above or below which, the infiltration time is higher. The tables also show 
that these three design variables (skin width, flange width, and blade height) do not vary with pressure, unlike the laminate thicknesses.

The optimal blade stacking sequence becomes $\left[90^{\circ}{ }_{80}\right]$ for all cases, as expected, since $90^{\circ}$ fibers are in the flow direction and provide the least resistance. Once again, the blade stacking sequence is insensitive to the compaction pressure. The "*" symbols next to the skin stacking sequences in the tables are to note that the permeability of the skin is always that perpendicular to the fibers, regardless of the fiber orientation. Therefore $\left[ \pm 45^{\circ}{ }_{10}\right]_{s}$ is fixed for all skin designs since it was the optimal structural stacking sequence.

Table 4.12 Manufacturing Optimum $(P=500 \mathrm{kPa} ; F=800 \mathrm{kN})$

\begin{tabular}{cc}
\hline Geometric Variables & \\
\hline$l_{s}$ & $81.20 \mathrm{~cm}$ \\
$l_{f}$ & $2.82 \mathrm{~cm}$ \\
$h_{b}$ & $0.05 \mathrm{~cm}$ \\
$t_{s}$ & $0.485 \mathrm{~cm}$ \\
$t_{b}$ & $0.971 \mathrm{~cm}$
\end{tabular}

Material Variables

$\begin{array}{ll}\boldsymbol{\Theta}_{s} & 22222^{*} \\ \boldsymbol{\Theta}_{b} & 3333333333\end{array}$

Manufacturing Variables

$\begin{array}{ll}P & 500 \mathrm{kPa} \\ T & 155^{\circ} \mathrm{C}\end{array}$

Structural Analysis

$\begin{array}{ll}F & 800 \mathrm{kN} \\ M & 5.07 \mathrm{~kg} \\ r_{\text {stiff }} & 60.82 \\ r_{\text {skin }} & 20.57 \\ l_{f} / t_{b} & 2.90 \\ l_{f} / l_{s} & 0.035\end{array}$

Manufacturing Analysis

$\begin{array}{ll}\tau & 7.43 \mathrm{~s} \\ \Lambda & 1.00\end{array}$

Table 4.13 Manufacturing Optimum $(P=700 \mathrm{kPa} ; F=800 \mathrm{kN})$

\begin{tabular}{cc}
\hline Geometric Variables & \\
\hline$l_{s}$ & $81.20 \mathrm{~cm}$ \\
$l_{f}$ & $2.82 \mathrm{~cm}$ \\
$h_{b}$ & $0.05 \mathrm{~cm}$ \\
$t_{s}$ & $0.479 \mathrm{~cm}$ \\
$t_{b}$ & $0.957 \mathrm{~cm}$
\end{tabular}

Material Variables

$\begin{array}{ll}\boldsymbol{\Theta}_{s} & 22222^{*} \\ \boldsymbol{\Theta}_{b} & 3333333333\end{array}$

Manufacturing Variables

$\begin{array}{ll}P & 700 \mathrm{kPa} \\ T & 159^{\circ} \mathrm{C}\end{array}$

Structural Analysis

$\begin{array}{ll}F & 800 \mathrm{kN} \\ M & 5.02 \mathrm{~kg} \\ r_{\text {stiff }} & 63.24 \\ r_{s k i n} & 21.12 \\ l_{f} / t_{b} & 2.95 \\ l_{f} / l_{s} & 0.035\end{array}$

Manufacturing Analysis

\begin{tabular}{ll}
$\tau$ & $5.15 \mathrm{~s}$ \\
$\Lambda$ & 1.00 \\
\hline
\end{tabular}


Table 4.14 Manufacturing Optimum $(P=900 \mathrm{kPa} ; F=800 \mathrm{kN})$

\begin{tabular}{cc}
\hline Geometric Variables & \\
\hline$l_{s}$ & $81.20 \mathrm{~cm}$ \\
$l_{f}$ & $2.82 \mathrm{~cm}$ \\
$h_{b}$ & $0.05 \mathrm{~cm}$ \\
$t_{s}$ & $0.474 \mathrm{~cm}$ \\
$t_{b}$ & $0.947 \mathrm{~cm}$
\end{tabular}

Material Variables

$\begin{array}{ll}\Theta_{s} & 22222^{*} \\ \Theta_{b} & 3333333333\end{array}$

Manufacturing Variables

$\begin{array}{ll}P & 900 \mathrm{kPa} \\ T & 161^{\circ} \mathrm{C}\end{array}$

Structural Analysis

$\begin{array}{ll}F & 800 \mathrm{kN} \\ M & 4.98 \mathrm{~kg} \\ r_{\text {stiff }} & 65.10 \\ r_{\text {skin }} & 21.54 \\ l_{f} / t_{b} & 2.98 \\ l_{f} / l_{s} & 0.035\end{array}$

Manufacturing Analysis

\begin{tabular}{ll}
$\tau$ & $3.94 \mathrm{~s}$ \\
$\Lambda$ & 1.00 \\
\hline
\end{tabular}

Table 4.15 Manufacturing Optimum $(P=1,100 \mathrm{kPa} ; F=800 \mathrm{kN})$

\begin{tabular}{cc}
\hline Geometric Variables & \\
\hline$l_{s}$ & $81.20 \mathrm{~cm}$ \\
$l_{f}$ & $2.82 \mathrm{~cm}$ \\
$h_{b}$ & $0.05 \mathrm{~cm}$ \\
$t_{s}$ & $0.470 \mathrm{~cm}$ \\
$t_{b}$ & $0.939 \mathrm{~cm}$
\end{tabular}

Material Variables

$\begin{array}{ll}\Theta_{s} & 22222^{*} \\ \Theta_{b} & 3333333333\end{array}$

Manufacturing Variables

$\begin{array}{ll}P & 1,100 \mathrm{kPa} \\ T & 163^{\circ} \mathrm{C}\end{array}$

Structural Analysis

$\begin{array}{ll}F & 800 \mathrm{kN} \\ M & 4.95 \mathrm{~kg} \\ r_{\text {stiff }} & 66.62 \\ r_{\text {skin }} & 21.87 \\ l_{f} / t_{b} & 3.00 \\ l_{f} / l_{s} & 0.035\end{array}$

Manufacturing Analysis

$\begin{array}{ll}\tau & 3.21 \mathrm{~s} \\ \Lambda & 1.00\end{array}$

The cure temperature, which was not needed in the structural optimization, now comes into play and is a function of the compaction pressure. The results show that higher pressures allow for higher temperatures, both of which contribute to the reductions in infiltration time. This reduction in fill time is from $7.43 \mathrm{~s}$ at $700 \mathrm{kPa}$, with a temperature of $155^{\circ} \mathrm{C}$, to $3.21 \mathrm{~s}$ at $1,100 \mathrm{kPa}$, with a temperature of $163^{\circ} \mathrm{C}$. The optimum temperature also serves as the upper bound above which the resin would gel prior to 
complete infiltration. Thus, increasing the baseline pressure of $700 \mathrm{kPa}$ to $1,100 \mathrm{kPa}$ decreases the fill time by nearly $38 \%$.

Although not used in the manufacturing optimization, the tables also list the results of the structural analysis for the manufacturing optima. Like the infiltration time, the panel mass decreases with compaction pressure from $5.07 \mathrm{~kg}$ at $500 \mathrm{kPa}$ to $4.95 \mathrm{~kg}$ at $1,100 \mathrm{kPa}$. However, note that the buckling constraints are severely violated. The geometric constraints, though, are satisfied.

Because the designs presented in Tables 4.12-4.15 are unable to support the applied load, their usefulness to the problem at hand is limited. The previous results indicate that the optimizer was attempting to reduce the panel to a flat plate of dimensions $W \times L \times t_{s}$ (i.e., the skin). Note that the small flange and blade are the result of constraints placed on the design variables due to the discrete nature of the genetic algorithm. The skin can be made to accept the desired loading without buckling by increasing its thickness. The optimization problem can, therefore, be reformulated using the number of plies in the skin $\left(N_{s}\right)$ and the cure temperature as the sole design variables. In accordance with the previous results, the stacking sequence of the resulting skin is taken to be of the form $\left[ \pm 45^{\circ}{ }_{N_{s} / 2}\right]_{s}$. The manufacturing optimizations can then be redone using this new approach.

Table 4.16 presents the manufacturing optima for constant loading ( $800 \mathrm{kN})$ at several compaction pressures. These results show that as $P$ increases, the optimal cure temperature also increases, and the infiltration time is reduced as was the case in the other 
manufacturing optima. Moreover, now that the skin thickness is allowed to vary, the number of plies in the skin has increased nearly threefold in all four cases to support the loading. The skin buckling constraint, therefore, is critical or nearly critical in all cases. The nearly critical cases are attributed to the fact that the value of $N_{s}$ varies in increments of four plies to satisfy the balanced and symmetric conditions. The increased skin thickness, as expected, also raises the fill times and panel mass from the previous results.

Table 4.16 Revised Manufacturing Optima $(F=800 \mathrm{kN})$

\begin{tabular}{ccccccc}
\hline $\boldsymbol{P}(\mathbf{k P a})$ & $\boldsymbol{N}_{\boldsymbol{s}}$ & $\boldsymbol{t}_{\boldsymbol{s}}(\mathbf{c m})$ & $\boldsymbol{T}\left({ }^{\circ} \mathbf{C}\right)$ & $\boldsymbol{r}_{\text {skin }}$ & $\boldsymbol{M}(\mathbf{k g})$ & $\tau(\mathbf{s})$ \\
\hline 500 & 112 & 1.36 & 149 & 0.999 & 13.7 & 22.3 \\
700 & 116 & 1.39 & 151 & 0.924 & 14.1 & 17.0 \\
900 & 116 & 1.37 & 154 & 0.942 & 13.9 & 12.9 \\
1,100 & 116 & 1.36 & 155 & 0.958 & 13.8 & 10.5 \\
\hline
\end{tabular}

Similar optimization data are shown for variable loading cases with constant pressure ( $700 \mathrm{kPa}$ ) in Table 4.17. As the load demand on the skin increases, so does the skin thickness, and consequently the mass and infiltration time. For constant pressure, the thicker skins also tend to reduce the optimal cure temperature, and once again, the buckling constraint is nearly critical. The extremes of optimal designs for the structures and manufacturing cases shown thus far illustrate that a multiobjective formulation of the problem is essential and is the subject of the next section.

Table 4.17 Revised Manufacturing Optima $(P=700 \mathrm{kPa})$

\begin{tabular}{ccccccc}
\hline $\boldsymbol{F}(\mathbf{k N})$ & $\boldsymbol{N}_{\boldsymbol{s}}$ & $\boldsymbol{t}_{\boldsymbol{s}}(\mathbf{c m})$ & $\boldsymbol{T}\left({ }^{\circ} \mathbf{C}\right)$ & $\boldsymbol{r}_{\text {skin }}$ & $\boldsymbol{M}(\mathbf{k g})$ & $\boldsymbol{\tau}(\mathbf{s})$ \\
\hline 600 & 104 & 1.24 & 154 & 0.961 & 12.6 & 12.2 \\
800 & 116 & 1.39 & 151 & 0.924 & 14.1 & 17.0 \\
1,000 & 124 & 1.48 & 150 & 0.945 & 15.0 & 20.8 \\
\hline
\end{tabular}




\subsubsection{Combined Structural and Manufacturing Optima}

Incorporating the new mass definition and geometric constraints, the reformulated optimization problem for the GA is given by

$$
\min \Psi=M^{* *}+\tau^{*}
$$

which replaces Eq. 3.17. The optimum design for the baseline compaction pressure and loading is given in Table 4.18. Comparing the geometry to that of the structural optimum for the same pressure and loading from Table 4.6 , it can be seen that the skin is now $2.4 \%$ narrower than before, while the flange width and blade height have grown by $8.6 \%$ and $5.0 \%$, respectively. To-scale drawings comparing the unit cell geometry of the combined optimum to those of the structural and manufacturing optima for the same pressure and loading case are shown in

Figure 4.16. Note in the figure that the manufacturing design (second row in Table 4.16) is left "open-ended" since it is a flat plate of width $81.2 \mathrm{~cm}$ and thus, has no unit cell.

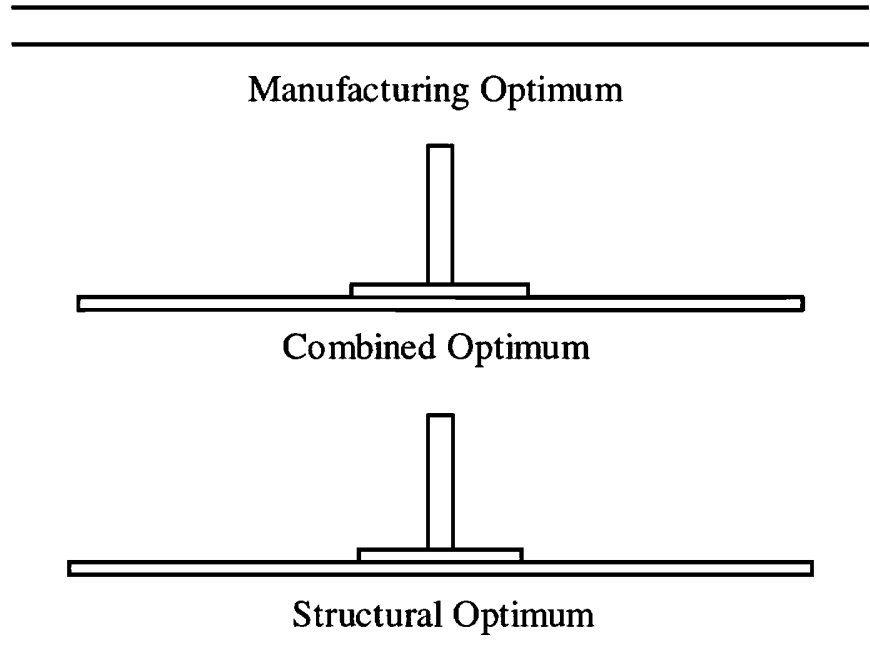

Figure 4.16 Comparison of Panel Designs $(P=700 \mathrm{kPa}, F=800 \mathrm{kN})$ 
The stacking sequence of the skin remains $\left[ \pm 45^{\circ}{ }_{10}\right]_{s}$ as in the structural optima since it does not affect the manufacturing analysis. The blade, however, has incorporated two $90^{\circ}$ stacks into the all-zero structural optimum in order to reduce its permeability and thus, reducing its infiltration time, as was the case in the first set of manufacturing optima. The addition of $90^{\circ}$ plies also accounts for the increase in the blade height since the stiffener modulus is less than that for the all-zero blade. The stacking sequence is described as $\left[0^{\circ}{ }_{32} / 90^{\circ}{ }_{8}\right]_{s}$ because the buckling model for the stiffener used in Chapter 2 depends only on the numbers of each ply orientation not on their order. Recall that Young's modulus for the stiffener (Eq. 2.49) is a function of the in-plane stiffnesses (Eq. 2.45), which are functions of the ply orientations through the lamination parameters. Note that the in-plane lamination parameters (Eq. 2.46) are independent of the stacking order as compared to the flexural lamination parameters (Eq. 2.48). The resulting stacking sequence given above causes the stiffener buckling ratio to approach its critical value, rather than the skin buckling ratio which was the case for the structural optima in Section 4.2.2. The ratio of flange width to blade thickness is also nearly critical. The other constraints, however, are satisfied.

The panel mass of the combined optimum in Table 4.18 is $3.4 \%$ higher than that of the structural optimum for the same loading and pressure from Table 4.6. Also compare the combined optimum infiltration time of $498 \mathrm{~s}$ to the $693 \mathrm{~s}$ achieved when the structural optimum was cured at the same cure temperature of $120^{\circ} \mathrm{C}$ as was shown in Table 4.11. Figure 4.17 plots the infiltration times over a range of temperatures for both the structural 
and combined optima. The figure shows that the optimal temperature for the combined case is indeed $120^{\circ} \mathrm{C}$, as was determined by the $\mathrm{GA}$, while the temperature that gives the smallest infiltration time $(678 \mathrm{~s})$ for the structural optimum is $118^{\circ} \mathrm{C}$. As in previous figures, the "•" symbols indicate the point at which the resin gels. These results show that the optimal cure temperature does not depend solely on the compaction pressure, but also on the values of the other design variables (i.e., geometry and stacking sequence). This again illustrates the need for a multidisciplinary approach to the design optimization problem.

Table 4.18 Combined Optimum $(P=700 \mathrm{kPa} ; F=800 \mathrm{kN})$

\begin{tabular}{cc}
\hline Geometric Variables & \\
\hline$l_{s}$ & $27.15 \mathrm{~cm}$ \\
$l_{f}$ & $6.66 \mathrm{~cm}$ \\
$h_{b}$ & $5.25 \mathrm{~cm}$ \\
$t_{s}$ & $0.479 \mathrm{~cm}$ \\
$t_{b}$ & $0.957 \mathrm{~cm}$
\end{tabular}

Material Variables

$\Theta_{s} \quad 22222$

$\Theta_{b} \quad 1111111133$

Manufacturing Variables

$\begin{array}{ll}P & 700 \mathrm{kPa} \\ T & 120^{\circ} \mathrm{C}\end{array}$

Structural Analysis

$\begin{array}{ll}F & 800 \mathrm{kN} \\ M & 7.91 \mathrm{~kg} \\ r_{\text {stiff }} & 0.994 \\ r_{\text {skin }} & 0.979 \\ l_{f} / t_{b} & 6.95 \\ l_{f} / l_{s} & 0.245\end{array}$

Manufacturing Analysis

$\tau \quad 498 \mathrm{~s}$

$\Lambda \quad 1.00$ 


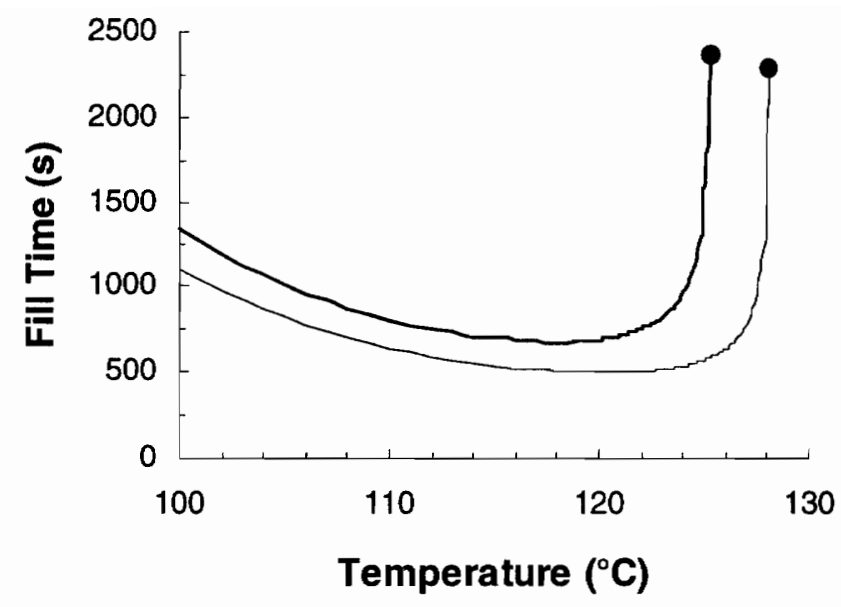

Figure 4.17 Effect of Temperature Variations in Optimization Results $(P=700 \mathrm{kPa}, F=800 \mathrm{kN})$

In order to verify that the design in Table 4.18 is indeed optimal, the design variables can be perturbed to observe the effects in the structural and manufacturing responses of the panel. The plots that follow, similar to the parametric studies in Section 4.1, demonstrate these resulting trade-offs between the manufacturing and structural objective functions. The infiltration time and the panel mass are used as the abscissa and the ordinate, respectively, and variations in a design variable will be shown as a contour.

Figure 4.18 shows the results of varying the skin width, keeping the other design variables fixed at their optimal values. From the figure, it can be seen that increases in $l_{s}$ decrease both the panel mass and fill time. The "๑" symbol again indicates that the resin has gelled, and the "o" symbol indicates the largest value $l_{s}$ can take. The "+" symbols indicate bounds for buckling constraint satisfaction. The upper bound is where the 
stiffener buckling ratio is critical, and the lower bound is where the skin buckling ratio is critical. The optimum value of $27.82 \mathrm{~cm}$ lies in the middle rather than at the lower bound due to the discrete nature of the genetic algorithm. The plot indicates that the lowest values of the two objectives occurs when $l_{s}=W$, but both buckling constraints are violated. Thus, the skin width computed by the GA is the optimum.

The effect of changing the blade height, while keeping the other design variables fixed, on the two objectives is similar to that of the skin width. The smallest infiltration time and panel mass occurs when the $h_{b}$ shrinks to zero. However, to satisfy both buckling constraints, the height must be at least $5.25 \mathrm{~cm}$, which again was the optimum value given in Table 4.18. Arrows are used to indicate the direction of increasing the margin for buckling constraint satisfaction.

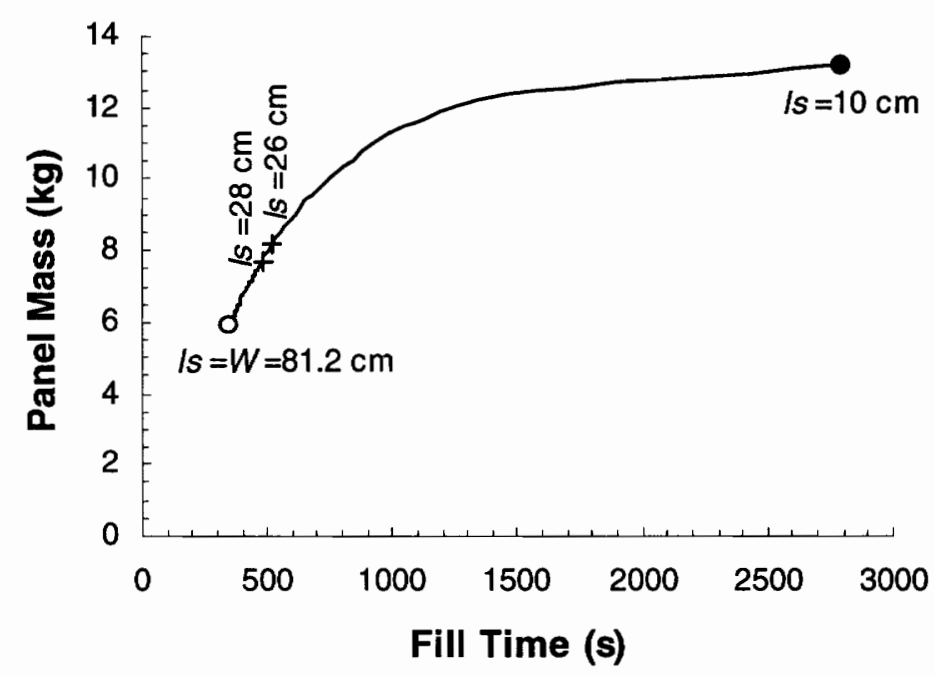

Figure 4.18 Objective Trade-off for Variable Skin Width 


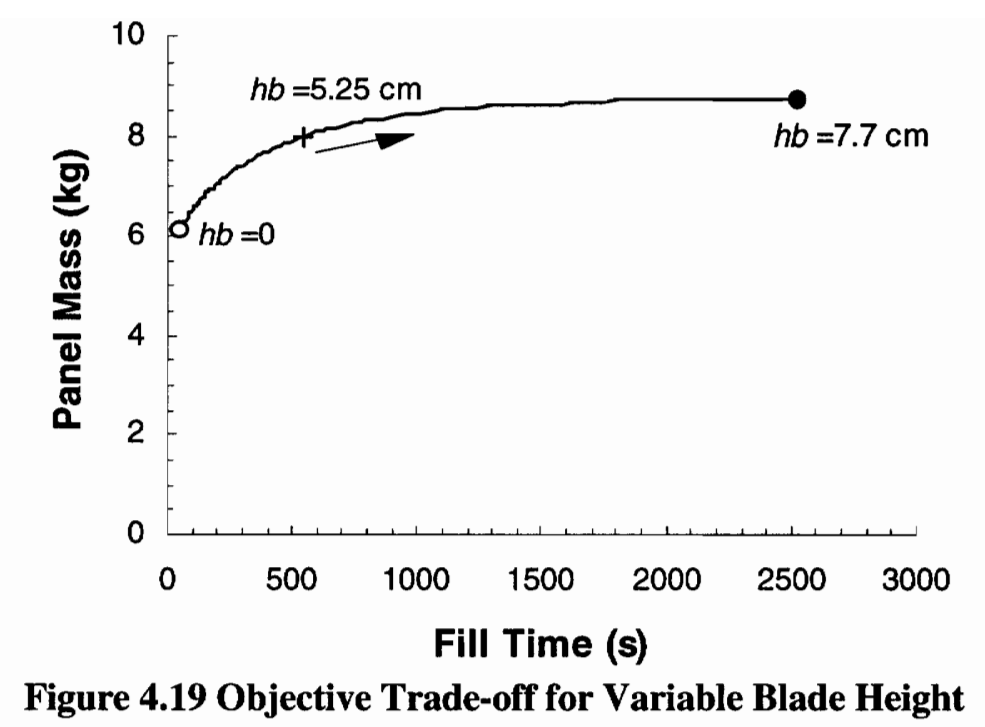

Varying the flange width, as shown in Figure 4.20, produces a different result. This time, the plot indicates an opposition in the objectives. The minimum-mass design occurs when $l_{f}=0.14 l_{s}(M=7.40 \mathrm{~kg})$. This design also has the largest infiltration time $(\tau=2,406 \mathrm{~s}$ ), and designs with smaller flanges cause the resin to gel prematurely. Moreover, this buckling constraints for this design are violated.

The minimum time design, on the other hand, does satisfy the buckling constraints and occurs when $l_{f}=0.64 l_{s}(\tau=299 \mathrm{~s}, M=9.82 \mathrm{~kg})$. Above this value, both the mass and time increase. This design, however, violates the geometric constraint that $l_{f} \leq 7 t_{b}$ with a value of $l_{f}=19 t_{b}$. The value of $l_{f}$ that satisfies all the constraints is $0.245 l_{s}$, giving the optimum design, as previously found, which is $19 \%$ lighter than the minimum time design, but fills $67 \%$ less quickly. It would appear that relaxing the geometric constraint would give a better design in terms of the manufacturing response, but recall that all other design 
variables are fixed. Thus, a change in the constraint would affect the outcome of the optimization and the resulting design.

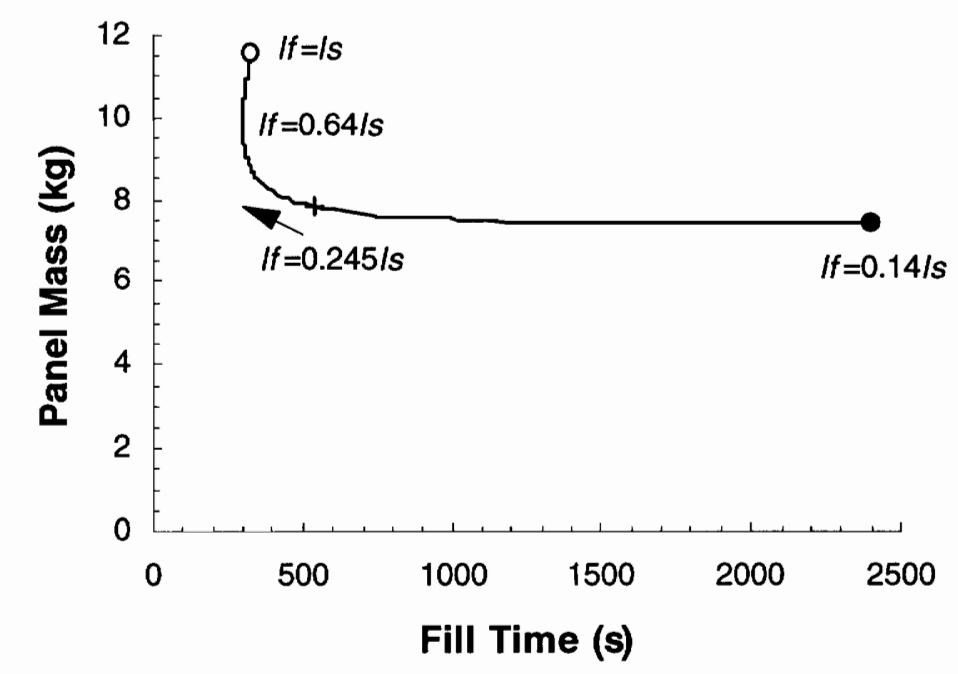

Figure 4.20 Objective Trade-off for Variable Flange Width

Figure 4.21 shows that compaction pressure has a common effect on the fill time and panel mass. As with the skin width and blade height, increasing the pressure decreases both the structural and manufacturing objectives, although the effect is more pronounced in the time rather than the mass. Note that premature gelation of the resin does not occur over the plotted pressure range, and that the pressure should be below $1,000 \mathrm{kPa}$ for the buckling constraints to be satisfied. This is the optimal pressure for the given geometry and cure temperature and yields a time and mass of $307 \mathrm{~s}$ and $7.82 \mathrm{~kg}$, respectively. Recall that variations in temperature have no direct impact on the structural response of the panel and that their effect on the manufacturing performance was already shown in Figure 4.17. 


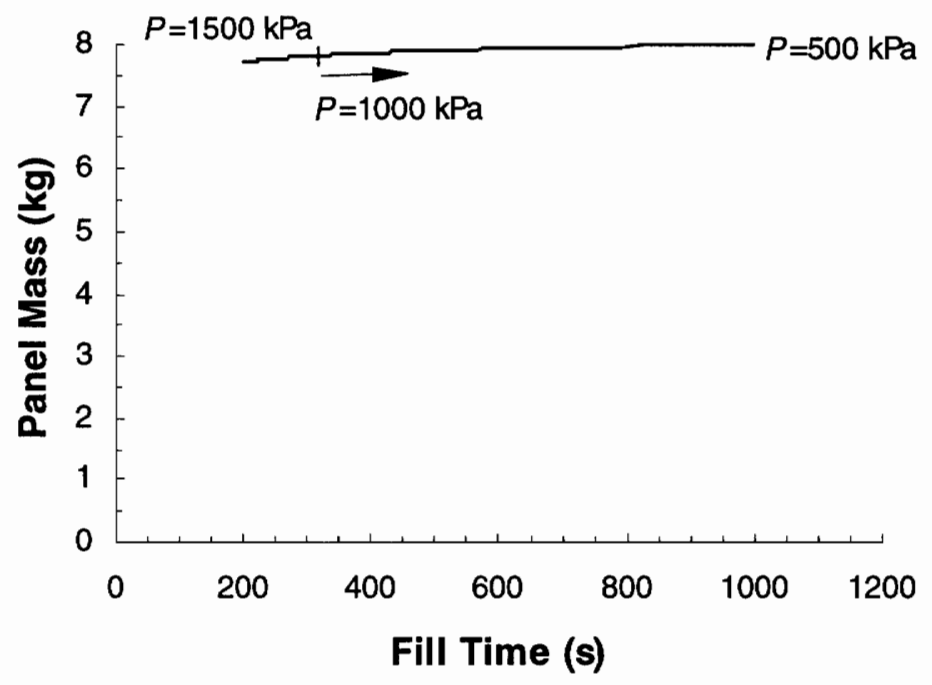

Figure 4.21 Objective Trade-off for Variable Pressure

It is now desired to discuss the optimization results for different values of pressure and loading. Table 4.19 shows the optimal design for an increased compaction pressure of $900 \mathrm{kPa}$. The values of the geometric design variables for this case are close to those of the $700 \mathrm{kPa}$ case in Table 4.18, differing only by at most $1.4 \%$ (flange width). The stacking sequences are unchanged from the $700 \mathrm{kPa}$ case. It is also worthy to point out that although the geometry is similar, the higher pressure has allowed the cure temperature to increase by $3^{\circ} \mathrm{C}$. The net result of these changes is to give a design that is only $0.38 \%$ lighter but fills $29 \%$ faster, while satisfying all the constraints.

To explain why the geometry does not vary with increased pressure, note that the ratio of flange width to blade thickness has essentially its critical value of 7 (6.94 due to the discrete nature of the GA). Figure 4.22 compares the values of the design variables for the combined optima at two additional pressures, $500 \mathrm{kPa}$ and $1,100 \mathrm{kPa}$, to the 700 $\mathrm{kPa}$ and $900 \mathrm{kPa}$ cases. The design variables in the figure are normalized with respect to 
those of the $700 \mathrm{kPa}$ case (Table 4.18). The plot shows that as the pressure increases, the temperature also increases, but there are only slight variations in the panel geometry. These results suggest that the optimizer tends to fix the flange width to its critical value (or as close as possible because of discretization, which accounts for the small variations) and then adjusts the skin width and blade height accordingly to support the desired load.

Table 4.19 Combined Optimum

$$
(P=900 \mathrm{kPa} ; F=800 \mathrm{kN})
$$

\begin{tabular}{cc}
\hline Geometric Variables & \\
\hline$l_{s}$ & $26.81 \mathrm{~cm}$ \\
$l_{f}$ & $6.57 \mathrm{~cm}$ \\
$h_{b}$ & $5.30 \mathrm{~cm}$ \\
$t_{s}$ & $0.474 \mathrm{~cm}$ \\
$t_{b}$ & $0.947 \mathrm{~cm}$
\end{tabular}

Material Variables

$\begin{array}{ll}\Theta_{s} & 22222 \\ \Theta_{b} & 1111111133\end{array}$

Manufacturing Variables

$P \quad 900 \mathrm{kPa}$

$T \quad 123^{\circ} \mathrm{C}$

Structural Analysis

$\begin{array}{ll}F & 800 \mathrm{kN} \\ M & 7.88 \mathrm{~kg} \\ r_{\text {stiff }} & 0.981 \\ r_{s k i n} & 0.973 \\ l_{f} / t_{b} & 6.94 \\ l_{f} / l_{s} & 0.245\end{array}$

Manufacturing Analysis

\begin{tabular}{ll}
$\tau$ & $352 \mathrm{~s}$ \\
$\Lambda$ & 1.00 \\
\hline
\end{tabular}

The mass and infiltration times for the four pressure cases are plotted in Figure

4.23. Due to the consistency of the geometry cited above, the panel mass remains fairly 
constant, with a slight decreasing trend from the added pressure. The increase in compaction pressure has a more profound effect on the infiltration time, however, with a $70 \%$ savings when going from $500 \mathrm{kPa}$ to $1,100 \mathrm{kPa}$.

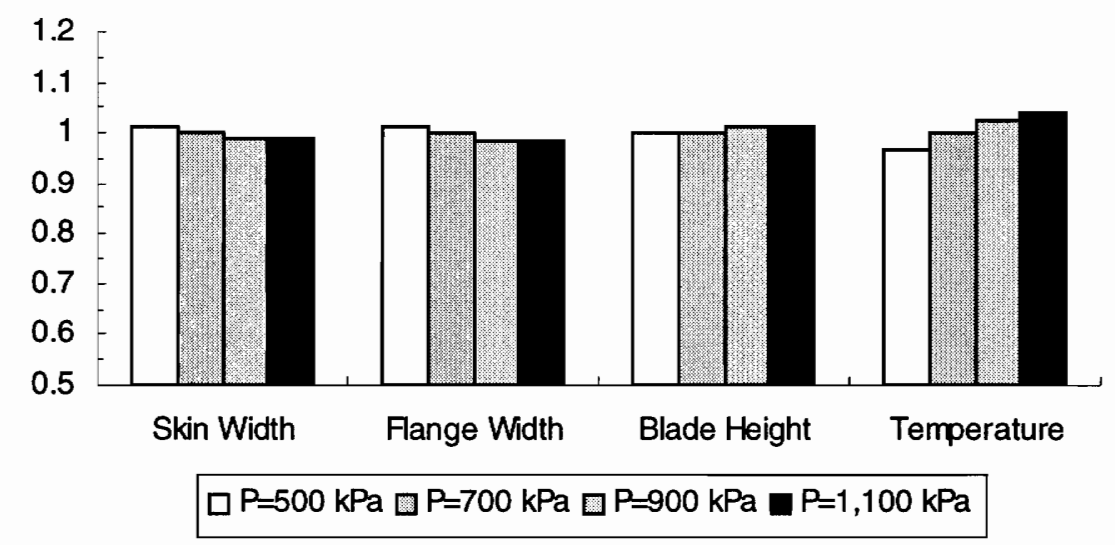

Figure 4.22 Comparison of Design Variables for Variable Pressure Combined Optima

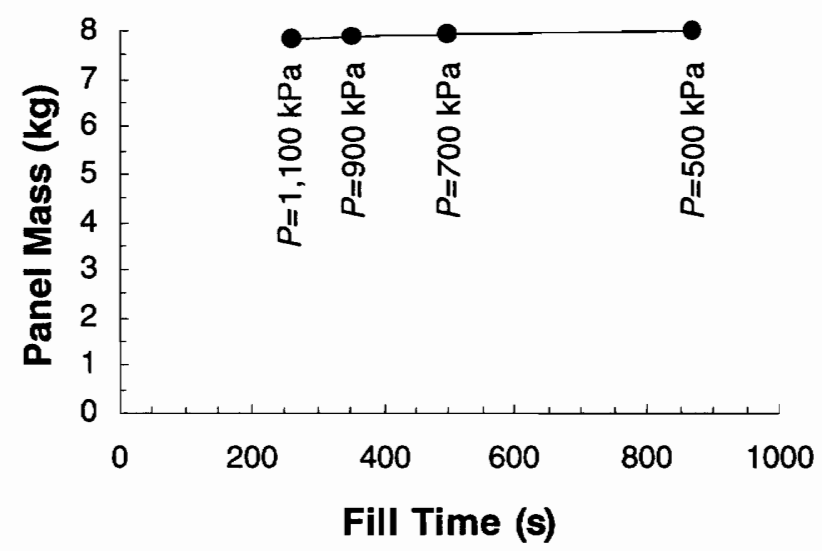

Figure 4.23 Combined Optima for Variable Compaction Pressure $(F=800 \mathrm{kN}$ )

The effect of increasing the applied loading is illustrated in Table 4.20. To accept the higher loading, the value of $l_{s}$ decreases, with $h_{b}$ and $l_{f}$ increasing. The stacking sequences are once more unchanged, and all constraints are satisfied. These changes raise the panel mass to $8.47 \mathrm{~kg}$. The cure temperature is also affected by the new geometry and drops to $118^{\circ} \mathrm{C}$. The infiltration time also pays a penalty of $35 \%$ from that in Table 4.18. 
Table 4.20 Combined Optimum $(P=700 \mathrm{kPa} ; F=1,000 \mathrm{kN})$

Geometric Variables

$\begin{array}{ll}l_{s} & 24.50 \mathrm{~cm} \\ l_{f} & 6.61 \mathrm{~cm} \\ h_{b} & 5.85 \mathrm{~cm} \\ t_{s} & 0.479 \mathrm{~cm} \\ t_{b} & 0.957 \mathrm{~cm}\end{array}$

Material Variables

$\begin{array}{ll}\Theta_{s} & 22222 \\ \Theta_{b} & 1111111133\end{array}$

Manufacturing Variables

$P \quad 700 \mathrm{kPa}$

$T \quad 118^{\circ} \mathrm{C}$

Structural Analysis

$\begin{array}{ll}F & 1,000 \mathrm{kN} \\ M & 8.47 \mathrm{~kg} \\ r_{\text {stiff }} & 0.983 \\ r_{s k i n} & 0.918 \\ l_{f} / t_{b} & 6.91 \\ l_{f} / l_{s} & 0.270\end{array}$

Manufacturing Analysis

$\tau \quad 673 \mathrm{~s}$

$\Lambda \quad 1.00$

Note again, that the change in the flange width is very small due to discretization. The design variable values for an additional loading case of $600 \mathrm{kN}$ are compared to those for the $800 \mathrm{kN}$ (Table 4.18), on which the values are normalized, and 1,000 kN (Table 4.20) cases in Figure 4.23. As before, it can be seen that the optimizer tends to fix the flange width to a value of seven times the blade thickness, the geometric constraint. Thus, to support the increasing load, the skin width and blade height must decrease and increase, respectively. The cure temperature experiences a small decline, but remains fairly constant. Figure 4.25 plots the objective function values for the three loading cases. The 
figure illustrates an apparently linear trend of decreasing panel mass and fill time with increasing load. When the load is reduced from $1,000 \mathrm{kN}$ to $600 \mathrm{kN}$, there is a savings of $41 \%$ and $12 \%$ in time and mass, respectively.

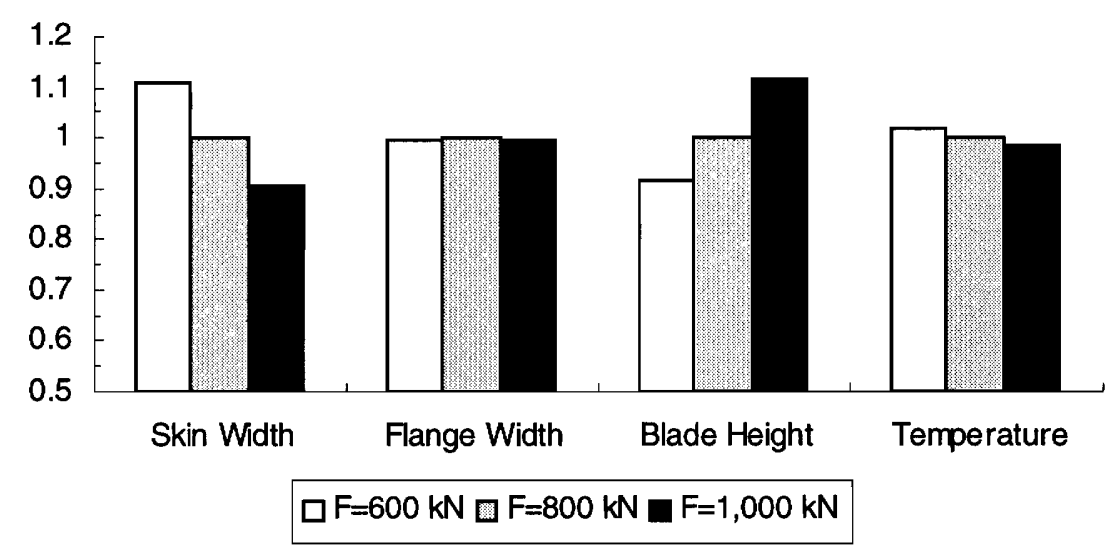

Figure 4.24 Comparison of Design Variables for Variable Loading Combined Optima

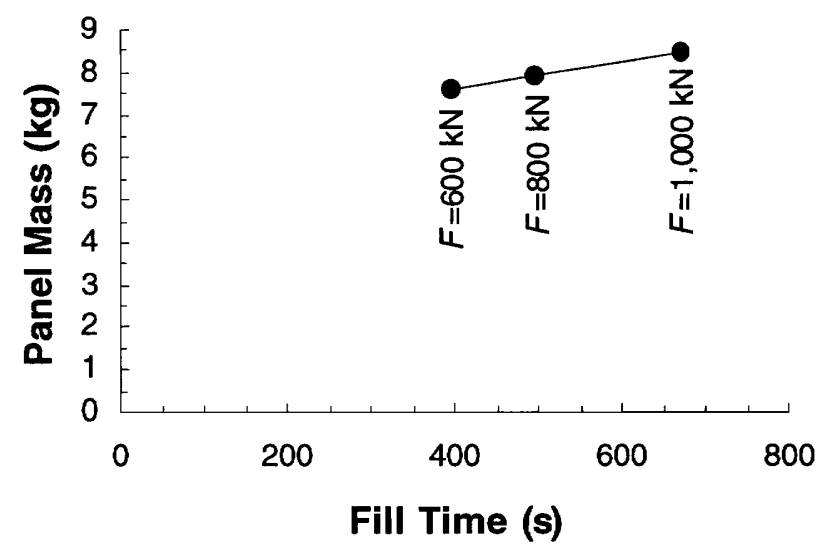

Figure 4.25 Combined Optima for Variable Loading $(P=700 \mathrm{kPa})$

Finally, it is desired to examine the trade-offs between the structural and manufacturing objectives in the optimal designs for three of the pressure/loading cases previously discussed. Figure 4.26 plots these optima. The triangles represent the structural optima, the circles represent the combined optima, and the diamonds represent 
the manufacturing optima. Note that the manufacturing optima are the ones from Tables 4.16 and 4.17 that can sustain the applied loading. The gray, filled, and open symbols are for the $P=700 \mathrm{kPa} / F=800 \mathrm{kN}$ (the baseline case), $P=900 \mathrm{kPa} / F=800 \mathrm{kN}, P=700$ $\mathrm{kPa} / F=1,000 \mathrm{kN}$ cases, respectively. The lines connecting the optima are used for illustration purposes. From the figure, it can be seen that the manufacturing optima have the lowest infiltration times, but also the highest panel masses. The structural optima, on the other hand, have the highest times, but the lowest masses. Recall that the infiltration times for the structural optima are computed at a temperature of $120^{\circ} \mathrm{C}$ as was shown in Table 4.11. For example, the difference in objectives between the manufacturing and structural optima for the baseline case, shown in gray, are $98 \%$ for the time and $84 \%$ for the mass. That is, the manufacturing optimum for the baseline pressure and loading fills 98\% faster than the structural optimum for the same case, but is $84 \%$ more massive.

The combined optima, as expected, essentially fall in between the structural and manufacturing optima, but lie closer to the structural designs. Going from the structural designs to the combined, the panel mass increases slightly in all cases, more so in the baseline case shown as gray symbols. However, compensating for the higher mass, the infiltration time decreases for all three cases and is most pronounced in the baseline and added loading (open symbols) cases, where the times decrease by approximately $200 \mathrm{~s}$ from those for the structural optima. These results indicate that there is a definite tradeoff between the manufacturing and structural responses in the design of a stiffened 
composite panel and that a multidisciplinary approach is essential to the optimization problem.

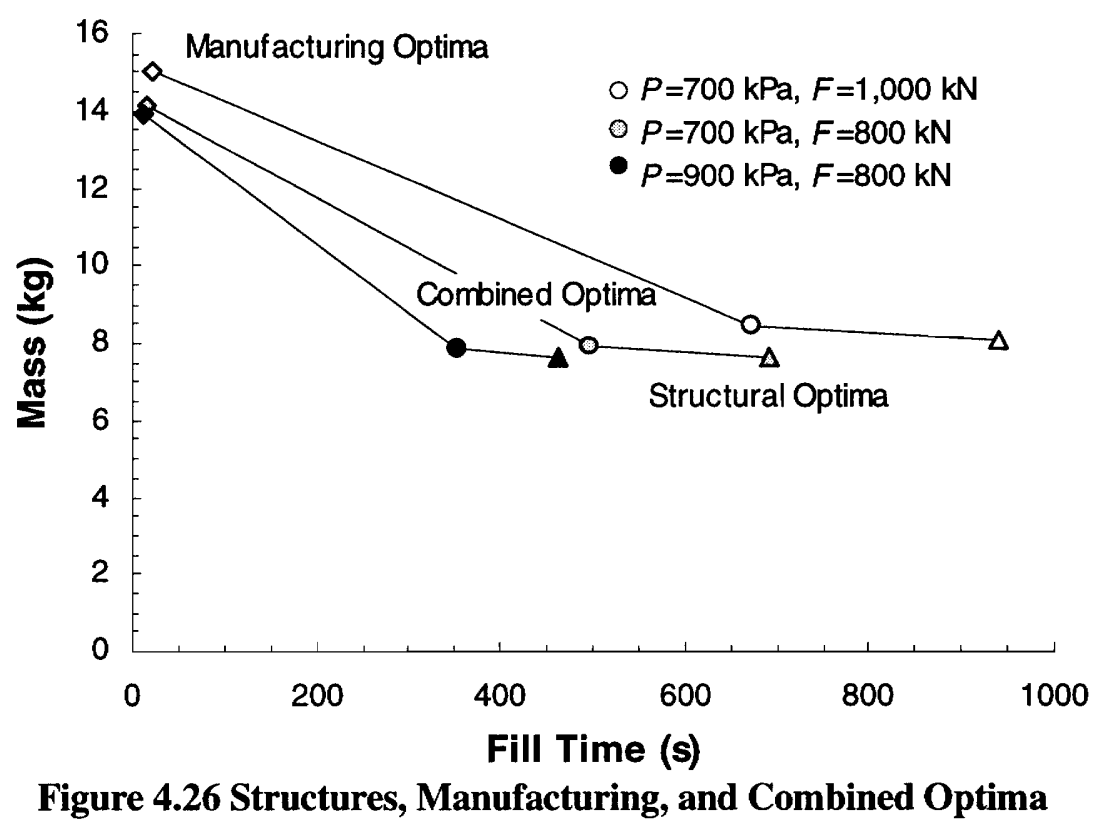

\subsection{Concluding Remarks}

To goal of this research was to integrate manufacturing considerations into the design optimization of a blade-stiffened panel. To this end, models were developed to determine the panel mass, the critical buckling loads, and the time for resin infiltration as measures of the structural and manufacturing responses. The design variables were selected as those that influence both disciplines and include the cross-sectional geometry, laminate stacking sequences, compaction pressure, and cure temperature.

Using a baseline design as a reference, each of the design variables were varied to observe these effects. A genetic algorithm was also used to generate optimum designs for the structures and manufacturing cases alone, as well as for the combined case. The 
results presented in this chapter have demonstrated a strong correlation between structures and manufacturing, confirming that the approach to the problem must be multidisciplinary if manufacturing is to be incorporated into the design process. Using this methodology, it is possible to observe the effects of structurally important design variables, such as geometry and material properties, on the manufacturing performance of the panel design and the less obvious effects of manufacturing process variables, such as pressure and temperature, on the structural performance. This approach also allows the trade-offs between manufacturing and structural performance to be studied. The optimization results indicate the structural and manufacturing objectives compete with each other depending on their relative importance. For example, panels designed solely for structural performance have the lowest masses but the highest infiltration times, while panels designed for manufacturing, on the other hand, have the lowest infiltration times but the highest masses. When both disciplines are given equal importance, the optimal design lies somewhere in between paying only a slight penalty in mass, but achieving a substantial savings in infiltration time.

Future considerations for continuing this research center primarily around the refinement of the analysis models used. As a first step, a more detailed structural analysis model can be used to analyze the buckling behavior of the panel as a whole without splitting it into two separate elements. This can be done using a panel analysis code, such as PASCO [50], or an empirical buckling model for stiffened panels. The refined structural analysis would more than likely eliminate the need for the "artificial" constraints 
placed on the geometry that were necessary with the simplified model. In addition, stacking sequences could play a larger role as design variables, which would impact both the structural and manufacturing analyses. From the manufacturing perspective, the effects of tooling materials and geometry and heat transfer can also be incorporated into the analysis model used, and eventually, the model can be extended to three-dimensions. Moreover, because industry is primarily concerned with cost, the results of the sciencebased manufacturing analysis can be expressed in terms of some cost parameter. This approach can also be taken with the structural analysis in the form of life cycle costs. Thus, the weight and infiltration time minimization problem can be transformed into an overall cost minimization. 


\section{REFERENCES}

1. L. Mesquita and M.P. Kamat, "Optimization of Stiffened Laminated Composite Plates with Frequency Constraints," Engineering Optimization 11, pp. 77-88 (1987).

2. S. Nagendra, R.T. Haftka, and Z. Gürdal, "Design of a Blade Stiffened Composite Panel with a Hole," Composite Structures 18, pp. 195-219 (1991).

3. S. Nagendra, R.T. Haftka, and Z. Gürdal, "Design of Blade Stiffened Composite Panels by a Genetic Algorithm Approach," 34th AIAA/ASME/AHS Structures, Structural Dynamics, and Materials Conference (La Jolla, CA), April 19-22, 1993, pp. 2418-2436.

4. S. Nagendra, D. Jestin, Z. Gürdal, R.T. Haftka, and L.T. Watson, "Improved Genetic Algorithm for the Design of Stiffened Composite Panels," 15th Intl. Symp. on Math. Prog. (Ann Arbor, MI), Aug. 15-19, 1994.

5. S. Nagendra, R.T. Haftka, and Z. Gürdal, "Buckling Optimization of Laminate Stacking Sequence with Strain Constraints," 10th Conference on Electronic Computation (Indianapolis, IN), 1991, pp. 205-212.

6. N. Kogiso, L.T. Watson, Z. Gürdal, and R.T. Haftka, "Genetic Algorithm with Local Improvement for Composite Laminate Design," ASME Winter Annual Meeting (New Orleans, LA), Nov. 29-Dec. 3, 1993.

7. R.T. Haftka and J.L. Walsh, "Stacking Sequence Optimization for Buckling of Laminated Plates by Integer Programming," AIAA Journal 3, pp. 814-819 (1992).

8. R. Le Riche, "Composite Structures Optimization by Genetic Algorithms," Ph.D. Dissertation. Virginia Polytechnic Institute and State University, Blacksburg, VA, 1994.

9. Le Riche and R.T. Haftka, "Optimization of Laminate Stacking Sequence for Buckling Load Maximization by Genetic Algorithm," AIAA Journal 5, 951-956 (1993).

10. J.L. Henderson, "Laminated Plate Design Using Genetic Algorithms and Parallel Processing," Computing Systems in Engineering 5, pp. 441-453 (1994).

11. W.T. Freeman, L.F. Vosteen, and S. Siddiqi, "A Unified Approach for Composite Cost Reporting and Prediction in the ACT Program," 1st NASA Advanced Composites Technology Conference (Seattle, WA), Oct. 29-Nov. 1, 1990, pp. 357369.

12. B.R. Norton, "Cost Drivers in Design and Manufacture of Composite Structures," Engineered Materials Handbook 1, pp. 419-427 (1993).

13. W. Freeman, L. Ilcewicz, G. Swanson, and T. Gutowski, "Designers' Unified Cost Model," 2nd NASA Advanced Composites Technology Conference (Lake Tahoe, NV), Nov. 4-7, 1991, pp. 27-45. 
14. H.T. Hahn, "Design for Manufacturability (DFM) for Composites," 8th International Conference on Composite Materials (Honolulu, HI), July 15-19, 1991, pp. 1-D-1 to 1-D-10.

15. B.G. Falzon and G.P Steven, "The Effect of Manufacturing Methods on the Buckling and Postbuckling Strength of Thin Skinned Fibre Composite Panels," International. Conference on Advanced Composite Materials (University of Wollongong, Australia), Feb. 15-19, 1993, pp. 239-246.

16. F.C. Robertson, "Resin Transfer Moulding of Aerospace Resins--A Review," British Polymer Journal 20, pp. 417-426 (1988).

17. J.P. Coulter, B.I. Smith, and S.I. Guceri, "Experimental and Numerical Analysis of Resin Impregnation During the Manufacturing of Composite Materials," Journal of Reinforced Plastics and Composites 9, pp. 201-217 (1988).

18. J.P. Coulter and S.I. Guceri, "Resin Impregnation During Composite Manufacturing: Theory and Experimentation," Composites Science and Technology 35, pp. 317-330 (1991).

19. S. Li and R. Gauvin, "Numerical Analysis of the Resin Flow in Resin Transfer Molding," Journal of Reinforced Plastics and Composites 10, pp. 314-327 (1991).

20. M.H. Weideman, A.C. Loos, H.B. Dexter, and G.H. Hasko, "An Infiltration/Cure Model for Manufacture of Fabric Composites by the Resin Infusion Process," Virginia Tech Center for Composite Materials and Structures Report No. CCMS-9205, Virginia Polytechnic Institute and State University, Blacksburg, VA, 1992.

21. A.C. Loos, J.D. MacRae, V.H. Hammond, K.E. Kranbuehl, S.M. Hart, G.H. Hasko, and A.M. Markus, "Analytical Modeling and Sensor Monitoring for Optimal Processing of Advanced Textile Structural Composites by Resin Transfer Molding," 3rd NASA Advanced Composites Technology Conference (Long Beach, CA), June 8-11, 1992, pp. 361-380.

22. J.D. MacRae, A.C. Loos, H.B. Dexter, J.W. Deaton, and G.H. Hasko, "Development and Verification of a Resin Film Infusion/Resin Transfer Molding Simulation Model for Fabrication of Advanced Textile Composites," VPI-E-94-09, Virginia Polytechnic Institute and State University, Blacksburg, VA, 1994.

23. J.C. Fingerson, A.C. Loos, and H.B. Dexter, "Verification of a Three-Dimensional Resin Transfer Molding Process Simulation Model," VPI E-95-04, Virginia Polytechnic Institute and State University, Blacksburg, VA, 1995.

24. G.S. Springer, "Resin Flow During the Cure of Fiber Reinforced Composites," Journal of Composite Materials 16, 400-410 (1982).

25. G.S. Springer, "A Model of the Curing Process of Epoxy Matrix Composites," Progress in Science and Engineering of Composites, Japan Society of Composite Materials, pp. 23-35 (1982).

26. A.C. Loos and G.S. Springer, "Calculation of Cure Process Variables During Cure of Graphite-Epoxy Composites," in Composite Materials, Quality Assurance and Processing, ASTM STP 797, pp. 110-118 (1983). 
27. E.W. Lee, P.R. Butzloff, and K.D. Sewell, "Predictive Manufacturing of V-22 Composite Grips," 50th AHS Annual Forum (Washington, DC), May 11-13, 1994, pp. 171-179.

28. B.A. Wilson, "Design/Tooling/Manufacturing Interfaces," Engineered Materials Handook 1, pp. 428-431 (1993).

29. M. Yoshimura and A. Kimura, "Evolutional Optimization of a Product Design Based on Concurrent Processing of Design and Manufacturing Information," 5th AIAA/USAF/NASA/ISSMO Symposium on Multidisciplinary Analysis and Optimization (Panama City Beach, FL), Sept. 7-9, 1994, pp. 434-442.

30. M. Woytowitz, M. Warburton, and J. Glatfelter, "Knowledge-Based Concurrent Engineering of Aircraft Structural Components," 50th AHS Annual Forum (Washington, DC), May 11-13, 1994, pp. 691-698.

31. W.J. Marx, D.N. Mavris, D.P. Schrage, "Integrating Design and Manufacturing for a High Speed Civil Transport Wing," 19th ICAS Congress (Anaheim, CA), Sept. 1823, 1994, pp. 3019-3028.

32. J.V. Busch and B. Poggiali, "Micro-Computer Based Cost Estimation for Composite Fabrication Processes," 31st International SAMPE Symposium and Exhibition (Los Angeles, CA), April 7-10, 1986, pp. 232-244.

33. R.L. Ramkumar, R.B. Vastava, and S.K. Saha, "Manufacturing Cost Model for Composites," 23rd International SAMPE Technical Conference, October, 1991, pp. 982-994.

34. S.L. Metschan, K.S. Wilden, G.C. Sharpless, and R.M Andelman, "Cost Model Relationships Between Textile Manufacturing Processes and Design Details for Transport Fuselage Elements," 3rd NASA Advanced Composites Technology Conference (Long Beach, CA), June 8-11, 1992, pp. 323-340.

35. T. Gutowski, D. Hoult, G. Dillon, E-T Neoh, S. Muter, E. Kim, and M. Tse, "Development of a Theoretical Cost Model for Advanced Composite Fabrication," Composites Manufacturing 5, pp. 231-239 (1994).

36. L.P. Chao, M.V. Gandhi, and B.S. Thompson, "A Design-for-Manufacturing Methodology for Incorporating Manufacturing Uncertainties in the Robust Design of Fibrous Laminated Composite Structures," Journal of Composite Materials 27, pp. 175-194 (1993).

37. B.P. Kristinsdottir and Z.B. Zabinsky, "Including Manufacturing Tolerances in Composite Design," 35th AIAA/ASME/ASCE/AHS/ASC Structures, Structural Dynamics, and Materials Conference (Hilton Head, SC), April 18-20, 1994, pp. 1413-1422.

38. H. Darcy, Les Fontaines Publiques de la Ville de Dijon, Dalmount, Paris, 1856.

39. P. Chiou and A. Letton, "Reaction Kinetics and Chemoviscosity of a Thermoset Exhibiting Complex Curing Behavior," 4th American Society for Composites Technical Conference, 1989, pp. 157-166.

40. J.D. Ferry, Viscoelastic Properties of Polymers, 2nd Edition, Wiley, New York, 1970. 
41. T.G. Gutowski, Z. Cai, S. Bauer, D. Boucher, J. Kingery, and S. Wineman, "Consolidation Experiments for Laminate Composites," Journal of Composite Materials 21, pp. 650-669, 1987.

42. P.C. Carman, "Fluid Flow through Granular Beds," Trans. Int. Chem. Eng. 15, pp. 150-166, 1937.

43. B.R. Gebart, "Permeability of Unidirectional Reinforcements for RTM," Journal of Composite Materials 26, pp. 1100-1133 (1992).

44. Z. Cai and D.J. Lawrie, "A Simplified Mold Filling Program for the RTM Process," 24th International SAMPE Technical Conference, October 20-22, 1992.

45. R.M. Jones, Mechanics of Composite Materials, Scripta Book Company, Washington, DC, 1975.

46. R.T. Haftka and Z. Gürdal, Elements of Structural Optimization, 3rd Revised and Expanded Edition, Kluwer Academic Publishers, Dordrecht, The Netherlands, 1992.

47. D.E. Goldberg, Genetic Algorithms in Search, Optimization, and Machine Learning, Addison-Wesley Publishing Company, Inc., Reading, MA, 1989.

48. L. Davis, ed., Handbook of Genetic Algorithms. Van Nostrand Reinhold, New York, 1991.

49. J.H. Holland, "Genetic Algorithms," Scientific American 1, pp. 66-72 (1992).

50. W.J. Stroud and M.S. Anderson, "PASCO: Structural Panel Analysis and Sizing Code: Capability and Analytical Foundations," NASA Technical Memorandum 80801, Nov. 1981. 


\section{VITA}

The author was born on November 16, 1972, at Eglin Air Force Base, Florida, to James and Angeles Henderson. Within a year, the family relocated to Langely Air Force Base in Hampton, Virginia. In the fall of 1977, the family settled in Newport News, Virginia, where the author attended elementary, middle, and high schools. In June of 1990, he graduated from Denbigh High School as class valedictorian. That fall, the author began his undergraduate studies at Virginia Tech in Blacksburg, Virginia, and received his Bachelor of Science degree in May of 1994 with summa cum laude distinction in Aerospace Engineering with a minor in mathematics. The summer following his freshman year, he interned with ST Systems Corporation, a NASA contractor in Hampton, Virginia. The next three summers were spent at NASA Langley Research Center, also in Hampton, under the Langley Aerospace Research Summer Scholars (LARSS) Program. The author then decided to further his education and received his Master of Science degree in Aerospace Engineering in July of 1996.

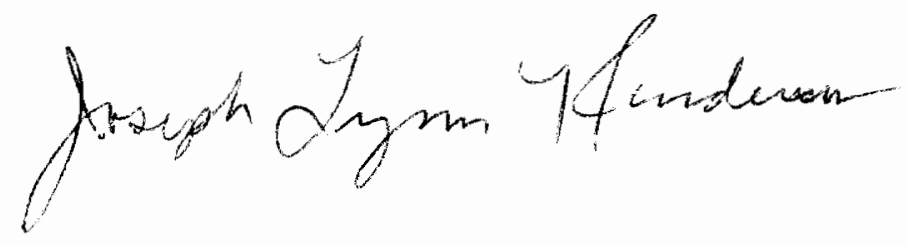

